\title{
The Ground State of a Gross-Pitaevskii Energy with General Potential in the Thomas-Fermi Limit
}

\author{
Georgia Karali \& Christos Sourdis
}

Communicated by C. DAFERMOS

\begin{abstract}
We study the ground state which minimizes a Gross-Pitaevskii energy with general non-radial trapping potential, under the unit mass constraint, in the ThomasFermi limit where a small parameter $\varepsilon$ tends to 0 . This ground state plays an important role in the mathematical treatment of recent experiments on the phenomenon of Bose-Einstein condensation, and in the study of various types of solutions of nonhomogeneous defocusing nonlinear Schrödinger equations. Many of these applications require delicate estimates for the behavior of the ground state near the boundary of the condensate, as $\varepsilon \rightarrow 0$, in the vicinity of which the ground state has irregular behavior in the form of a steep corner layer. In particular, the role of this layer is important in order to detect the presence of vortices in the small density region of the condensate, to understand the superfluid flow around an obstacle, and it also has a leading order contribution in the energy. In contrast to previous approaches, we utilize a perturbation argument to go beyond the classical Thomas-Fermi approximation and accurately approximate the layer by the Hastings-McLeod solution of the Painlevé-II equation. This settles an open problem (cf. Aftalion in Vortices in Bose Einstein Condensates. Birkhäuser Boston, Boston, 2006, pg. 13 or Open Problem 8.1), answered very recently only for the special case of the model harmonic potential (Gallo and Pelinovsky in Asymptot Anal 73:53-96, 2011). In fact, we even improve upon previous results that relied heavily on the radial symmetry of the potential trap. Moreover, we show that the ground state has the maximal regularity available, namely it remains uniformly bounded in the $\frac{1}{2}$-Hölder norm, which is the exact Hölder regularity of the singular limit profile, as $\varepsilon \rightarrow 0$. Our study is highly motivated by an interesting open problem posed recently by AFTALION, JERRARD, and RoYo-LETELIER (J Funct Anal 260:2387-2406 2011), and an open question of Gallo and PelinovsKy (J Math Anal Appl 355:495-526, 2009), concerning the removal of the radial symmetry assumption from the potential trap.
\end{abstract}




\section{Contents}

1. Introduction . . . . . . . . . . . . . . . . . . . 440

1.1. The Problem . . . . . . . . . . . . . . . . . . . . . . . . . . . . . 440

1.2. Motivation for the Current Work . . . . . . . . . . . . . . . . . . 442

1.2.1 Minimization of a Gross-Pitaevskii Energy Describing a Bose-Einstein

Condensate in a Potential Trap . . . . . . . . . . . . . . . 443

1.2.2 Semi-classical States of the Defocusing Nonlinear Schrödinger Equation 444

1.2.3 Applications to Related Problems . . . . . . . . . . . . . . . . . . . 447

1.3. Known Results . . . . . . . . . . . . . . . . . . . . . . . . . . . . . . 448

1.4. Statement of the Main Result . . . . . . . . . . . . . . . . . . . . . . 450

1.5. Outline of the Proof and Structure of the Paper . . . . . . . . . . . . . . . 455

2. Notation . . . . . . . . . . . . . . . . . . . . . . 457

3. Proof of the Main Result . . . . . . . . . . . . . . . . . . . . . . . 457

3.1. Setup Near the Curve . . . . . . . . . . . . . . . . . . . . . . . . . . . 457

3.2. Set Up Away from the Curve . . . . . . . . . . . . . . . . . . . . . . . . 464

3.3. The Approximate Solution $u_{\mathrm{ap}} \ldots \ldots \ldots$. . . . . . . . . . . . . 467

3.4. Mapping Properties of the Linearized Operator . . . . . . . . . . . . . . . . 469

3.5. Existence of a Solution . . . . . . . . . . . . . . . . . . . . . . 472

3.6. Proof of the Main Theorem . . . . . . . . . . . . . . . . . . . . . . . . 477

4. Further Properties of the Ground State $\eta_{\varepsilon} \ldots \ldots$. . . . . . . . . . . 483

5. Refined Estimates for the Auxiliary Functions $\xi_{\varepsilon}, f_{\varepsilon}$ in the Case of Radial Symmetry 491

6. Open Problems and Future Directions . . . . . . . . . . . . . . . 495

\section{Introduction}

\subsection{The Problem}

This paper is concerned with the analysis of the $\varepsilon \rightarrow 0$ limiting behavior of the Gross-Pitaevskii energy

$$
G_{\varepsilon}(u)=\int_{\mathbb{R}^{2}}\left\{\frac{1}{2}|\nabla u|^{2}+\frac{1}{4 \varepsilon^{2}}|u|^{4}+\frac{1}{2 \varepsilon^{2}} W(\mathbf{y})|u|^{2}\right\} \mathrm{d} \mathbf{y},
$$

minimized in

$$
\mathscr{H} \equiv\left\{u \in W^{1,2}\left(\mathbb{R}^{2} ; \mathbb{C}\right): \int_{\mathbb{R}^{2}} W(\mathbf{y})|u|^{2} \mathrm{~d} \mathbf{y}<\infty, \quad \int_{\mathbb{R}^{2}}|u|^{2} \mathrm{~d} \mathbf{y}=1\right\},
$$

where $\varepsilon>0$ is a small parameter and, unless specified otherwise, the potential $W$ will satisfy:

$$
W \text { is nonnegative, } \quad W \in C^{1} \text {, }
$$

and there exist constants $C>1, p \geqq 2$ such that

$$
\frac{1}{C}|\mathbf{y}|^{p} \leqq W(\mathbf{y}) \leqq C|\mathbf{y}|^{p} \quad \text { if }|\mathbf{y}| \geqq C,
$$

(see also Remark 2 below). It is common to refer to the above problem as the minimization of $G_{\varepsilon}$ under the unit mass constraint. 
Let $\lambda_{0}>\inf _{\mathbb{R}^{2}} W(\mathbf{y})$ be uniquely determined from the relation

$$
\int_{\mathbb{R}^{2}}\left(\lambda_{0}-W(\mathbf{y})\right)^{+} \mathrm{d} \mathbf{y}=1,
$$

where throughout this paper we will denote $f^{+} \equiv \max \{f, 0\}$. The choice of the value one in the above relation is dictated by the constraint (2), see also (13) below. We further assume that the region

$$
\mathscr{D}_{0} \equiv\left\{\mathbf{y} \in \mathbb{R}^{2}: W(\mathbf{y})<\lambda_{0}\right\}
$$

is a simply connected bounded domain, containing the origin, with smooth boundary $\partial \mathscr{D}_{0}$, such that

$$
\frac{\partial W}{\partial \mathbf{n}}>0 \text { on } \partial \mathscr{D}_{0}
$$

where $\mathbf{n}=\mathbf{n}(\mathbf{y})$ denotes the outward unit normal vector to $\partial \mathscr{D}_{0}$. This last assumption can be viewed as a non-degeneracy condition. We point out that these hypotheses admit physically relevant examples, used to model certain experiments (see the next subsection). We stress that the simply connectedness assumption is assumed for convenience purposes only (see Remark 1 below), and so is the fact that the setting is two-dimensional (see Remark 16 below).

It follows from [186, Prop. 1] (see also $[10,125,152]$ ) that the functional $G_{\varepsilon}$ has a unique real valued minimizer $\eta_{\varepsilon}>0$ in $\mathscr{H}$ (all complex valued minimizers are of the form $\eta_{\varepsilon} \mathrm{e}^{i \alpha}$, where $\alpha$ is a constant). The function $\eta_{\varepsilon}$ satisfies

$$
-\Delta \eta_{\varepsilon}+\frac{1}{\varepsilon^{2}} \eta_{\varepsilon}\left(W(\mathbf{y})+\eta_{\varepsilon}^{2}\right)=\frac{1}{\varepsilon^{2}} \lambda_{\varepsilon} \eta_{\varepsilon}, \eta_{\varepsilon}>0 \quad \text { in } \mathbb{R}^{2}, \quad \eta_{\varepsilon} \rightarrow 0 \quad \text { as }|\mathbf{y}| \rightarrow \infty,
$$

where $\frac{1}{\varepsilon^{2}} \lambda_{\varepsilon}$ is the Lagrange multiplier, which is also necessarily unique. The point being that (4) ensures that minimizing sequences of $G_{\varepsilon}$ in $\mathscr{H}$ cannot have their mass escaping at infinity (see also [178]); in fact the imbedding $\mathscr{H} \hookrightarrow L^{2}\left(\mathbb{R}^{2}, \mathbb{C}\right.$ ) is compact (see [125,208], or more generally [27, Lemma 3.1]). (One can also ignore the mass constraint, and instead minimize the functional $G_{\varepsilon}(u)-\frac{\lambda}{2 \varepsilon^{2}}\|u\|_{L^{2}(\mathbb{R})}^{2}$, with $\lambda>\min _{\mathbb{R}^{2}} W$, in which case the minimizer would satisfy (8) with $\lambda_{\varepsilon}=\lambda$.)

The real issue is the study of the asymptotic behavior of the minimizer $\eta_{\varepsilon}$ (or more generally of the critical points) of $G_{\varepsilon}$ as the parameter $\varepsilon$ tends to zero. Following AfTALION and Rivière [4], letting

$$
A=\lambda_{0}-W,
$$

the functional $G_{\varepsilon}$ can be rewritten as

$$
\begin{aligned}
G_{\varepsilon}(\eta)= & \int_{\mathbb{R}^{2}}\left\{\frac{1}{2}|\nabla \eta|^{2}+\frac{1}{4 \varepsilon^{2}}\left(\eta^{2}-A^{+}\right)^{2}+\frac{1}{2 \varepsilon^{2}} A^{-} \eta^{2}\right\} \mathrm{d} \mathbf{y} \\
& +\frac{1}{2 \varepsilon^{2}}\left(\lambda_{0}-\frac{1}{2} \int_{\mathbb{R}^{2}}\left(A^{+}\right)^{2} \mathrm{~d} \mathbf{y}\right)
\end{aligned}
$$


if $\eta \in \mathscr{H}$ is real valued, where $A^{+} \equiv \max \{A, 0\}$ and $A^{-} \equiv-\min \{A, 0\}$. Let $G_{\varepsilon}^{1}(\eta)$ denote the first integral above. Since $\eta_{\varepsilon}$ clearly minimizes $G_{\varepsilon}^{1}$ in $\mathscr{H}$, by constructing a suitable competitor based on $\sqrt{A^{+}}$, it is easy to see that

$$
G_{\varepsilon}^{1}\left(\eta_{\varepsilon}\right) \leqq C|\ln \varepsilon|,
$$

for some constant $C>0$, provided $\varepsilon$ is small (see [8,10,130], and Remark 18 herein). (We remark that the logarithmic term appears because (6), (7) imply that $\nabla\left(\sqrt{A^{+}}\right)$is not square-integrable near $\partial \mathscr{D}_{0}$.) Hence, for small $\varepsilon>0$, we have

$$
\int_{\mathbb{R}^{2}}\left\{\left(\eta_{\varepsilon}^{2}-A^{+}\right)^{2}+A^{-} \eta_{\varepsilon}^{2}\right\} \mathrm{d} \mathbf{y} \leqq C \varepsilon^{2}|\ln \varepsilon|,
$$

which suggests that $\eta_{\varepsilon}^{2}$ should be close, in some sense, to $A^{+}$as $\varepsilon \rightarrow 0$. Indeed, it can be shown that

$$
\eta_{\varepsilon} \rightarrow \sqrt{A^{+}} \text {uniformly in } \mathbb{R}^{2} \text { as } \varepsilon \rightarrow 0,
$$

see the references in Section 1.3 below. Therefore, loosely speaking, the minimizer $\eta_{\varepsilon}$ develops a steep corner layer along $\partial D_{0}$, as $\varepsilon \rightarrow 0$ [recall (6), (7)]. Note also that $\sqrt{A^{+}}$is the global minimizer of the "limit" functional

$$
G_{\varepsilon}^{0}(\eta)=\int_{\mathbb{R}^{2}}\left\{\frac{1}{4 \varepsilon^{2}} \eta^{4}+\frac{1}{2 \varepsilon^{2}} W(\mathbf{y}) \eta^{2}\right\} \mathrm{d} \mathbf{y},
$$

among real functions such that $\|\eta\|_{L^{2}\left(\mathbb{R}^{2}\right)}=1$ and $W \eta^{2} \in L^{1}\left(\mathbb{R}^{2}\right)$. In the context of Bose-Einstein condensates, see the following subsection, the function $\sqrt{A^{+}}$is known as the Thomas-Fermi approximation.

The estimates that are available in the literature for the convergence in (13), see Section 1.3 below, fail to encapsulate important information which is often required in interesting applications (see Section 1.2 below). As an illustrative example, let us mention that a lower bound for $\eta_{\varepsilon}$, sufficient to imply that the second variation $\partial^{2} G_{\varepsilon}\left(\eta_{\varepsilon}\right)$ is coercive (this is easy to prove but hard to estimate), does not seem to be known (in Remark 30 we will establish a spectral bound for $\partial^{2} G_{\varepsilon}\left(\eta_{\varepsilon}\right)$, and as a matter of fact one may even calculate sharp constants). Our main goal in this paper is to provide crucial details, missing from the known results that describe the statement (13) quantitatively, placing special emphasis on how $\eta_{\varepsilon}$ converges to the "singular limit" $\sqrt{A^{+}}$near $\partial \mathscr{D}_{0}$, as $\varepsilon \rightarrow 0$, and proving that it converges in a self-similar fashion as conjectured in [9]. Although it is a variational problem, our approach will be based more on partial differential equation and functional analysis tools. Our treatment is concise and systematic, and can be used to treat in a unified manner problems with similar features.

As will be apparent from one glance in the references of this paper and the following discussion, the current interest in the minimizer $\eta_{\varepsilon}$, and in problems that have it on their background, is phenomenal.

\subsection{Motivation for the Current Work}

The motivation for the current work is threefold: 


\subsubsection{Minimization of a Gross-Pitaevskii Energy Describing a Bose-Einstein} Condensate in a Potential Trap Among the many experiments on Bose-Einstein condensates (which we abbreviate BEC), one consists in rotating the trap holding the atoms in order to observe a superfluid behavior: the appearance of quantized vortices (see the books $[9,176,177]$ and the references that follow). This takes place for sufficiently large rotational velocities. On the contrary, at low rotation, no vortex is detected in the bulk of the condensate. In a BEC, all the atoms occupy the lowest energy state so that they can be described by the same complex valued wave function. The latter is at the same time the macroscopic quantum wave function of the condensate and minimizes a Gross-Pitaevskii type energy. A vortex corresponds to zeroes of the wave function with phase around it. In two dimensions, the GrossPitaevskii energy considered in $[5,10,125,126]$ has the form:

$$
E_{\varepsilon}(v)=\int_{\mathbb{R}^{2}}\left\{\frac{1}{2}|\nabla v|^{2}+\frac{1}{4 \varepsilon^{2}}|v|^{4}+\frac{1}{2 \varepsilon^{2}} W(\mathbf{y})|v|^{2}-\Omega \mathbf{y}^{\perp} \cdot(i v, \nabla v)\right\} \mathrm{d} \mathbf{y}, \quad v \in \mathscr{H},
$$

where $\Omega$ is the angular velocity, $\mathbf{y}=\left(\mathbf{y}_{1}, \mathbf{y}_{2}\right), \mathbf{y}^{\perp}=\left(-\mathbf{y}_{2}, \mathbf{y}_{1}\right), \varepsilon>0$ is a small parameter that corresponds to the Thomas-Fermi approximation [93,195], the trapping potential $W$ belongs in the class described in the previous subsection, and $(i v, \nabla v)=i v \nabla v^{*}-i v^{*} \nabla v$. It is clear that $\eta_{\varepsilon}$ is the unique real valued minimizer of $E_{\varepsilon}$ in $\mathscr{H}$.

For mathematical studies in the case where the condensate has an annular shape, we refer to [8] and [65], whilst for studies in a three-dimensional setting to [13,25], and [209]. For numerics we refer to the review article [30].

The density of the condensate is significant in $\mathscr{D}_{0}$ [keep in mind (13)], which is typically a disc or an annulus, and gets exponentially small outside of this domain. The case of harmonic trapping potential

$$
W\left(\mathbf{y}_{\mathbf{1}}, \mathbf{y}_{\mathbf{2}}\right)=\mathbf{y}_{\mathbf{1}}^{2}+\Lambda^{2} \mathbf{y}_{\mathbf{2}}^{2},
$$

for a fixed parameter $0<\Lambda \leqq 1$, has been considered in experiments in [158,159]. In recent experiments, in which a laser beam is superimposed upon the magnetic trap holding the atoms, the trapping potential $W$ is of a different type [181,192,204]:

$$
W(r)=r^{2}+a \mathrm{e}^{-b r^{2}}, \quad r^{2}=\mathbf{y}_{\mathbf{1}}^{2}+\mathbf{y}_{\mathbf{2}}^{2}, \quad a, b>0 .
$$

(By choosing $a, b$ accordingly, the domain $\mathscr{D}_{0}$ is either a disc or an annulus.)

The energy $E_{\varepsilon}$ bears a formal resemblance to the well-studied Ginzburg-Landau functional

$$
J_{\varepsilon}(u)=\int_{\mathbb{R}^{2}}\left\{\frac{1}{2}|\nabla u|^{2}+\frac{1}{4 \varepsilon^{2}}\left(|u|^{2}-1\right)^{2}-\Omega \mathbf{y}^{\perp} \cdot(i u, \nabla u)\right\} \mathrm{d} \mathbf{y},
$$

used to model superconductivity [39] (with $\Omega=0$ ), and superfluidity [14,188]. In their influential monograph [39], Bethuel, Brezis and Hélein have developed the main tools for studying vortices in "Ginzburg-Landau type" problems. As we have already pointed out, the singular behavior of $\sqrt{A^{+}}$near $\partial \mathscr{D}_{0}$ induces a cost of order 
$|\ln \varepsilon|$ in the energy. This causes a mathematical difficulty in detecting vortices by energy methods, since any vortex has precisely the same cost (see [39]). In other words, the possible presence of vortices will be hidden by the energetic cost of the corner layer. This difficulty is common in problems of Ginzburg-Landau type when the zero Dirichlet boundary condition is imposed (see for instance [188]). Fortunately, this difficulty can be surpassed in an elegant way by an idea that goes back to the work of Lassoued and Mironescu [148], and André and Shafrir [23]. By a remarkable identity, for any $v$, the energy $E_{\varepsilon}(v)$, for any $\Omega$, splits into two parts, the energy $G_{\varepsilon}\left(\eta_{\varepsilon}\right)$ of the density profile and a reduced energy of the complex phase $w=v / \eta_{\varepsilon}$ :

$$
E_{\varepsilon}(v)=G_{\varepsilon}\left(\eta_{\varepsilon}\right)+F_{\varepsilon}(w),
$$

where

$$
F_{\varepsilon}(w)=\int_{\mathbb{R}^{2}}\left\{\frac{\eta_{\varepsilon}^{2}}{2}|\nabla w|^{2}+\frac{\eta_{\varepsilon}^{4}}{4 \varepsilon^{2}}\left(|w|^{2}-1\right)^{2}-\eta_{\varepsilon}^{2} \Omega \mathbf{y}^{\perp} \cdot(i w, \nabla w)\right\} \mathrm{d} \mathbf{y},
$$

(see also [125]). In particular, the potential $W$ only appears in $G_{\varepsilon}$. In (18), the term $G_{\varepsilon}\left(\eta_{\varepsilon}\right)$ carries the energy of the singular layer near $\partial D_{0}$, and thus one may detect vortices from the reduced energy $F_{\varepsilon}$ by applying the Ginzburg-Landau techniques to the energy

$$
\tilde{F}_{\varepsilon}(w)=\int_{\mathbb{R}^{2}}\left\{\frac{\eta_{\varepsilon}^{2}}{2}|\nabla w|^{2}+\frac{\eta_{\varepsilon}^{4}}{4 \varepsilon^{2}}\left(|w|^{2}-1\right)^{2}\right\} \mathrm{d} \mathbf{y} .
$$

The difficulty will arise in the small density region of the condensate, namely $\mathbb{R}^{2} \backslash \mathscr{D}_{0}$, where $\eta_{\varepsilon}$ is small. This kind of splitting of the energy is by now standard in the rigorous analysis of functionals such as $E_{\varepsilon}$ (see also [154]). It clearly brings out the need for the study of the minimizer $\eta_{\varepsilon}$ of $G_{\varepsilon}$, which is the subject of the current work. In particular, as will also be apparent from the discussion in Section 1.3 below, estimating $\eta_{\varepsilon}$ near $\partial \mathscr{D}_{0}$ is essential for adapting the powerful Gamma-convergence techniques, developed for $J_{\varepsilon}$ (see [183] and the references therein), to the study of $E_{\varepsilon}$ (concerning issues of vortices, vortex lines [14], etc.). Obtaining these delicate estimates, without imposing any symmetry assumptions on the trapping potential, is the main contribution of the present paper.

\subsubsection{Semi-classical States of the Defocusing Nonlinear Schrödinger Equation}

Elliptic problems of the form (8) arise directly when seeking standing wave solutions

$$
u(\mathbf{y}, t)=\mathrm{e}^{-i \lambda t / \varepsilon} \eta(\mathbf{y})
$$

for the famous nonlinear Schrödinger equation (NLS):

$$
i \varepsilon \frac{\partial u}{\partial t}+\varepsilon^{2} \Delta u-W(\mathbf{y}) u \pm|u|^{q-1} u=0, \quad \mathbf{y} \in \mathbb{R}^{N}, t>0, u: \mathbb{R}^{N} \rightarrow \mathbb{C},
$$

where $N \geqq 1$ and $q>1$ (in the plus sign case, the potential $W$ may vary from that described previously). [See also (212) below.] For small $\varepsilon>0$, these standing-wave 
solutions are referred to as semi-classical states. The plus sign in (21) gives rise to the focusing NLS (attractive nonlinearity), while the minus sign to the defocusing NLS (repulsive nonlinearity) which is also known as the Gross-Pitaevskii equation (GP). It is also quite common to use the name Gross-Pitaevskii equation if (21) has a nonzero potential $W$, and the name nonlinear Schrödinger equation if $W \equiv 0$. Keep in mind that potentials of quadratic growth, as $|\mathbf{y}| \rightarrow \infty$, are the highest order potentials for local well-posedness of (21), see [169].

At low enough temperature, neglecting the thermal and quantum fluctuations, a Bose condensate can be represented by a complex wave function $u(\mathbf{y}, t)$ that obeys the dynamics of the NLS equation, see the excellent review article [57] and the references that follow. In particular, solutions of (8) provide, via (20), standing wave solutions for the GP equation with $N=2, q=3$. Let us mention that the minimizer of $G_{\varepsilon}$, considered in the entire space or in a bounded domain with zero boundary conditions (as in Remark 17 below), also plays an important role in the study of multi-component BECs (see $[133,155]$ and the references therein); in the dynamics of vortices confined in $\mathscr{D}_{0}$ under the flow of the Gross-Pitaevskii equation (see [138]); in the construction of traveling wave solutions with a stationary or traveling vortex ring to the Gross-Pitaevskii equation (see [180,202,203]); in the study of excited states of the GP equation (see $[119,174]$ ), and in Bose-Einstein condensates with weak localized impurities (see [100]).

In considering typical BEC experiments and in exploring the unprecedented control of the condensates through magnetic and optical "knobs", a mean-field theory is applied to reduce the quantum many-atom description to a scalar nonlinear Schrödinger equation (see [152]). The NLS equation is a well established model in optical and plasma physics as well as in fluid mechanics, where it emerges out of entirely different physical considerations [1,193]. In particular, for instance in optics, it emerges due to the so-called Kerr effect, where the material refractive index depends linearly on the intensity of incident light. The widespread use of the NLS equation stems from the fact that it describes, to the lowest order, the nonlinear dynamics of envelope waves.

Ground state solutions of the NLS are standing wave solutions, of the form (20), such that $\eta$ is positive, $\eta \in W^{1,2}\left(\mathbb{R}^{N}\right)$, and satisfies

$$
\varepsilon^{2} \Delta \eta-(W(\mathbf{y})-\lambda) \eta \pm|\eta|^{q-1} \eta=0 \text { in } \mathbb{R}^{N}, \eta \rightarrow 0 \text { as }|\mathbf{y}| \rightarrow \infty .
$$

The condition $u \in W^{1,2}\left(\mathbb{R}^{N}\right)$ is required to obtain solutions with physical interest. [Sometimes we will refer to positive solutions $\eta$ of (22) as ground states of (21).] In the subcritical case where $1<q<\frac{N+2}{N-2}$ if $N \geqq 3, q>1$ if $N=1,2$, ground states of the defocusing equation (22) _ correspond to global minimizers of $\mathscr{G}_{-}$(these are nontrivial if $\varepsilon>0$ is sufficiently small [125], see also [21, Example 5.11] and [82, Lemma 2.1]), while ground states of the focusing equation $(22)_{+}$correspond to mountain passes of $\mathscr{G}_{+}$, where

$$
\mathscr{G}_{ \pm}(\eta)=\int_{\mathbb{R}^{N}}\left\{\frac{\varepsilon^{2}}{2}|\nabla \eta|^{2}+(W(\mathbf{y})-\lambda) \frac{\eta^{2}}{2} \mp \frac{|\eta|^{q+1}}{q+1}\right\} \mathrm{d} \mathbf{y},
$$

in $W^{1,2}\left(\mathbb{R}^{N}\right)$ with $\int_{\mathbb{R}^{N}} W(\mathbf{y}) \eta^{2} \mathrm{~d} \mathbf{y}<\infty$ (see for instance $\left.[20,178]\right)$. 
In the focusing case, following the pioneering work of FLOER and WEINSTEIN [98], there have been enormous investigations on spike layer solutions for problem $(22)_{+}$, for small $\varepsilon>0$, typically under the conditions $\lambda<\inf _{\mathbb{R}^{N}} W(\mathbf{y})<$ $\liminf _{|\mathbf{y}| \rightarrow \infty} W(\mathbf{y})$, and $1<q<\frac{N+2}{N-2}$ if $N \geqq 3, q>1$ if $N=1,2$. The "critical" case, where $\inf _{\mathbb{R}^{N}} W(\mathbf{y})=\lambda$, has also received attention, see [47]. Actually, the latter case is related to the discussion following Definition 1 below. We refer the interested reader to $[20,78]$, and the references therein.

In the defocusing case (22) the previous subsection with $\lambda$ in place of $\lambda_{0}$, we see that the corresponding limit algebraic equation, obtained by formally letting $\varepsilon=0$ in (22) _, has the compactly supported continuous solution

$$
\eta_{0}=\left[(\lambda-W)^{+}\right]^{\frac{1}{q-1}} .
$$

Obviously, there is also the solution $-\eta_{0}$, and the trivial one. In fact, if $q>2$ then the "singular limit" $\eta_{0}$ is merely Hölder continuous with exponent $1 /(q-1)$ [recall (6), (7)]. In particular, it is easy to see that if $q \geqq 3$ then $\nabla \eta_{0}$ is not squareintegrable near $\partial \mathscr{D}_{0}$. If $q=2$ and $\eta>0$, then (22) scalar logistic equation [56], and $\eta_{0}$ is Lipschitz continuous. If $1<q<2$, then $\eta_{0}$ is at least differentiable. In the language of bifurcation theory [128], the solution set of the corresponding limit algebraic equation to (22) _ undergoes a supercritical pitchfork bifurcation at $\partial \mathscr{D}_{0}$ if $q>2$; a transcritical bifurcation if $q=2$; a subcritical pitchfork bifurcation if $1<q<2$. Notice also that $\eta_{0}$ is asymptotically stable (as an approximate equilibrium) with respect to the parabolic dynamics that correspond to (22). The question whether $\eta_{0}$ perturbs, for small $\varepsilon$, to a solution $\eta_{\varepsilon}$ of $(22)_{-}$, and keeping track of the maximal regularity available (to be defined in a moment), is a source of current mathematical interest. Note that, if $q \geqq 2$, such a solution would have a corner layer along $\partial \mathscr{D}_{0}$. The bifurcation that occurs at $\partial_{D_{0}}$ takes the problem off from the classical setting, where the roots of the corresponding algebraic equation (for fixed $\mathbf{y}$ ) are non-intersecting (see [77]).

The following definition is adapted from [53]:

Definition 1. Let $\alpha \in(0,1]$ be the largest number such that $\left\|\eta_{0}\right\|_{C^{0, \alpha}\left(\mathbb{R}^{N}\right)}<\infty$, we say that a solution $\eta_{\varepsilon}$ has maximal Hölder regularity if $\left\|\eta_{\varepsilon}\right\|_{C^{0, \alpha}\left(\mathbb{R}^{N}\right)}$ remains uniformly bounded as $\varepsilon \rightarrow 0$.

If $N=1$, problem (22) _ can be rewritten as a homoclinic connection problem for a 3-dimensional slow-fast system of ordinary differential equations [131]. At the points that correspond to $\partial \mathscr{D}_{0}$ we have loss of normal hyperbolicity of the corresponding slow manifold, due to a pitchfork or transcritical bifurcation. This fact prohibits the use of standard geometric singular perturbation theory $[92,131]$ in order to deduce the persistence of the "singular homoclinic orbit" $\eta_{0}$, for small $\varepsilon>0$. At present, much work in geometric singular perturbation theory deals with such situations, often using the "blowing-up" construction [145]. This approach has been successfully applied recently in [190] in a heteroclinic connection problem for a 4-dimensional slow-fast Hamiltonian system, sharing similar features with (22)_ with $q=3$, arising from the study of crystalline grain boundaries (see also 
$[18,97,191])$. Let us mention that there is an abundance of non-hyperbolic points in applications, see for instance [145], that have been successfully treated using this approach. In particular, singularly perturbed one-dimensional second-order elliptic systems, involving loss of normal hyperbolicity, arise in the study of the Dafermos regularization for singular shocks $[66,189]$. Although elegant, the arguments of this approach are intrinsically one-dimensional.

Elliptic systems where the singular limit has merely Hölder or Lipschitz regularity typically describe phase separation or spatial segregation, and have attracted a lot of current mathematical research, see $[53,63,199]$ and the references therein. These types of problems have been tackled in the latter references either by weak convergence arguments, yielding weak convergence in the Sobolev space $W^{1,2}$ and strong in $L^{2}$ (see also [69]), or the method of upper and lower solutions, yielding uniform convergence (see also [46,123,127]), as $\varepsilon \rightarrow 0$. The question of maximal regularity of the convergence to the singular limit profile is then addressed using a delicate analysis, based on monotonicity properties, blow-up techniques and Liouville-type theorems (see also Remark 23 below). To the best of our knowledge, for these systems, the maximal regularity property has only been proven in onedimensional cases, see [36]. In the case at hand, since $\nabla \eta_{0}$ is not square-integrable near $\partial \mathscr{D}_{0}$ if $q \geqq 3$, it is not clear how to use standard weak convergence arguments. Furthermore, it seems to be hard to construct a good pair of upper and lower solutions (especially) near $\partial \mathscr{D}_{0}$. A motivation for the current work is to show that the perturbation approach to such problems, we initiated in [134,135], can be adapted to treat problem (22) _ with general potential. We emphasize that the perturbation method seems to be the only one available at the moment that yields the maximal regularity property in higher-dimensional singular perturbation problems. (See the main theorem of [135], and Corollary 4 herein.) Among other advantages of the perturbation approach is that it provides finer estimates which, as we already stated, imply the maximal regularity property, it be applied in the study of non-minimizing solutions in systems of equations which in general lack the maximum principle (see assumption (B4) in [45]), and in supercritical problems that cannot be treated variationally (see for example Remark 35). Most importantly, for the problem at hand, it applies equally well without the assumption of radial symmetry on the equation, see the discussion in the following subsection.

1.2.3. Applications to Related Problems A strong motivation behind the current work is the possibility of adapting our approach to treat other interesting problems with similar features.

Variational problems of a similar type to (15), with spatially varying coefficients, have also been introduced to model vortex pinning due to material inhomogeneities in a superconductor (see the references in [12], [83, Sec. 6] and [183, Subsecs. 14.1.4, 14.3.5]). In [22], the authors considered the case where the corresponding pinning potential $\mathscr{W}(\mathbf{y})$ is nonnegative but vanishes at a finite number of points (see also [194]). Minimizers such that $\left|v_{\varepsilon}\right| \rightarrow \sqrt{\mathscr{W}}$ in $W^{1,2}$, as $\varepsilon \rightarrow 0$, were analyzed by variational methods. In their result, it was important that $\sqrt{\mathscr{W}}$ is in $W^{1,2}$, which is not the case here (also recall the previous discussion concerning weak convergence methods). In that context, the minimizer has corner layer 
behavior at points rather than curves. It would be of interest to study this type of problems from the perturbation viewpoint of the present paper which, in particular, does not require that the singular limit belongs in $W^{1,2}$.

A singularly perturbed elliptic equation of the form (50) below, considered in a bounded domain with zero boundary conditions, where the corresponding limit algebraic equation admits a fold bifurcation at the boundary of the domain, appears in the proof of the Lazer-Mckenna conjecture for a superlinear elliptic problem of Ambrosetti-Prodi type, see [71,73]. In that case, the corresponding minimizer (without the mass constraint) has a steep corner layer along the boundary of the domain, see also the old paper [197]. This situation is qualitatively similar to the problem considered here. In [71], by adapting variational techniques from [70], the behavior of the minimizer, as $\varepsilon \rightarrow 0$, was estimated in compact sets away from the boundary of the domain. We believe that, employing the perturbation techniques of the present paper, one can obtain fine estimates for the minimizer all the way up to the boundary. In turn, these could potentially lead to the construction of new type of solutions on top of the minimizer, for example solutions having small peaks near the boundary, or bifurcating from symmetry (as in [134,135]). Another reason for developing perturbation arguments for these problems is that unstable solutions are hard to find or describe accurately through purely variational methods, see Remark 35 for more details.

\subsection{Known Results}

In this subsection we gather some known properties of the real valued minimizer $\eta_{\varepsilon}$ of $G_{\varepsilon}$ in $\mathscr{H}$, under the assumptions described in Section 1.1. These have been studied in various contexts (see for instance $[8,10,65,125,163]$ ). As we will see in this paper, some are actually far from optimal.

The corresponding Lagrange multiplier satisfies, for small $\varepsilon>0$, the estimate:

$$
\left|\lambda_{\varepsilon}-\lambda_{0}\right| \leqq C|\ln \varepsilon|^{\frac{1}{2}} \varepsilon
$$

The real valued minimizer $\eta_{\varepsilon}$ satisfies the following estimates:

$$
\begin{aligned}
& 0<\eta_{\varepsilon} \leqq \sqrt{A^{+}}+C \varepsilon^{\frac{1}{3}} \text { in } \mathbb{R}^{2}, \\
& \eta_{\varepsilon}(\mathbf{y}) \leqq C \varepsilon^{\frac{1}{3}} \exp \left\{-c \varepsilon^{-\frac{2}{3}} \operatorname{dist}\left(\mathbf{y}, \partial \mathscr{D}_{0}\right)\right\} \quad \text { in } \mathbb{R}^{2} \backslash \mathscr{D}_{0},
\end{aligned}
$$

where $\mathbf{y} \mapsto \operatorname{dist}\left(\mathbf{y}, \partial \mathscr{D}_{0}\right)$ denotes the Euclidean distance in $\mathbb{R}^{2}$ from $\mathbf{y}$ to $\partial \mathscr{D}_{0}$,

$$
\left|\eta_{\varepsilon}-\sqrt{A^{+}}\right| \leqq C \varepsilon^{\frac{1}{3}} \sqrt{A^{+}} \quad \text { if } \quad \mathbf{y} \in \mathscr{D}_{0} \quad \text { and } \operatorname{dist}\left(\mathbf{y}, \partial \mathscr{D}_{0}\right) \geqq \varepsilon^{\frac{1}{3}}
$$

and

$$
\left\|\nabla \eta_{\varepsilon}\right\|_{L^{\infty}\left(\mathbb{R}^{2}\right)} \leqq C \varepsilon^{-1}
$$

for some constants $c, C>0$, if $\varepsilon$ is sufficiently small. Relation (25) follows from (11), see [10,125]. Relations (26), (27) have been shown in [125] (see also [163, 
Lemma B.1]) by constructing a suitable upper-solution of (8), and using the uniqueness of positive solutions of the latter equation in bounded domains with zero boundary conditions [43]. Relation (28) can be traced back to [8], and follows from lower- and upper-solution arguments in Equation (8) based on [23] (also keep in mind Remark 3 in [8]). Note that, in particular, estimates (26)-(28) yield (13). Lastly, estimate (29) on the gradient follows from the equation and a GagliardoNirenberg type inequality as in [38] (see Lemma 1 herein).

In the special case where the potential trap $W$ is additionally assumed to be radially symmetric, it follows from its uniqueness that the real valued minimizer $\eta_{\varepsilon}>0$ of $G_{\varepsilon}$ in $\mathscr{H}$ is also radially symmetric. In particular, if $\mathscr{D}_{0}$ is a ball of radius $R$, it has been shown recently in [10] that

$$
\eta_{\varepsilon}^{\prime}(r) \leqq 0 \quad \text { in }\left(R-\delta_{0}, R+\delta_{0}\right),
$$

for some small constant $\delta_{0}>0$, if $\varepsilon$ is small. The radial symmetry was used heavily by the authors of [10] in order to establish (30), using a maximum principle due to Berestycki, Nirenberg, and Varadhan [35] (see also the discussion following (79) herein) together with an intersection-comparison type of argument, mostly taking advantage of (7). The importance of the positivity of $\eta_{\varepsilon}$ and the radial symmetry of $W$ in deriving (30) can be naively seen from the following consideration. If $W^{\prime}(r) \geqq 0$ for all $r>0$, using that $\eta_{\varepsilon}>0$ and the method of moving planes [108], we can infer that $\eta_{\varepsilon}^{\prime}(r) \leqq 0$ for all $r>0$ (see also Proposition 2.1 in [152] for an approach via a radially-symmetric rearrangement argument which takes advantage of the minimizing character of $\eta_{\varepsilon}$, or Lemma 2 in [49] which uses the stability of $\eta_{\varepsilon}$ ). We point out that it is not clear how the aforementioned arguments apply in the case where $W$ is radially symmetric but the set $\left\{W-\lambda_{0}<0\right\}$ is an annulus, considered in $[8,13]$. Relation (30) was used in an essential way in [10] for estimating uniformly the auxiliary function

$$
f_{\varepsilon}(r) \equiv \xi_{\varepsilon}(r) / \eta_{\varepsilon}^{2}(r), \quad \text { where } \xi_{\varepsilon}(r) \equiv \int_{r}^{\infty} s \eta_{\varepsilon}^{2}(s) \mathrm{d} s,
$$

near the circle $|\mathbf{y}|=R$. The function $f_{\varepsilon}$ plays a crucial role in the study of the functional $E_{\varepsilon}$, see [10] and Section 5 below. (Actually, estimate (30) was needed in a region of the form $\left(R-\delta, R+C \varepsilon^{\frac{2}{3}}\right)$ for some constants $\delta, C>0$.) Making use of the previously mentioned estimates on $f_{\varepsilon}$ near the boundary of $\mathscr{D}_{0}$, and of some new estimates away from $\mathscr{D}_{0}$, the authors of [10] proved that if the angular velocity $\Omega$ is below a critical speed

$$
\Omega_{c}=\omega_{0}|\ln \varepsilon|-\omega_{1} \ln |\ln \varepsilon|,
$$

for some constants $\omega_{0}, \omega_{1}>0$, and $\varepsilon$ is sufficiently small, then the rotation has absolutely no effect on the minimizer. In other words, all minimizers $v_{\varepsilon}$ of $E_{\varepsilon}$ in $\mathscr{H}$ are of the form $v_{\varepsilon}=\eta_{\varepsilon} \mathrm{e}^{i \alpha}$ in $\mathbb{R}^{2}$, where $\alpha$ is a constant. In particular, at low velocity, there are no vortices in the condensate. We remark that this last assertion was previously known to hold true only in the bulk of the condensate, see [125]. It was left as an interesting open problem in [10] to see to what extent their analysis continues to hold if the assumption of radial symmetry on $W$ is dropped (see also 
Open Problem 8.1 in [9], and the open questions in the presentation [103]). Our results on the minimizer $\eta_{\varepsilon}$, which hold without any symmetry assumption on $W$, may represent a major step in the answering of this question.

Let us close this subsection by mentioning that the case where the potential is homogeneous of some order $s>0$, that is, $W(\lambda \mathbf{y})=\lambda^{s} W(\mathbf{y})$ for all $\lambda>0, \mathbf{y} \in \mathbb{R}^{2}$ [see (16) for an example with $s=2$ ], and locally Hölder continuous has been studied in the work of E. H. Lieb and his collaborators in $[152,153]$. By employing scaling and variational arguments, it was shown in the latter references that, as $\varepsilon \rightarrow 0$, the minimizer $\eta_{\varepsilon}$ converges to $\sqrt{A^{+}}$in the strong $L^{2}\left(\mathbb{R}^{2}\right)$ sense. In the special case of the model harmonic potential, described by (16) with $\Lambda=1$, a complete analysis has been carried in [104] (see the discussion following the statement of our main theorem for more details). By generalizing the divergence-free WKB method, a uniformly valid approximation for the condensate density of an ultra-cold Bose gas confined in a harmonic trap, that extends into the classically forbidden region (near and outside of $\partial \mathscr{D}_{0}$ ), has been established very recently in [182].

\subsection{Statement of the Main Result}

In order to state our main result, we need some definitions.

Let

$$
a_{\varepsilon}(\mathbf{y})=\lambda_{\varepsilon}-W(\mathbf{y}), \quad \mathbf{y} \in \mathbb{R}^{2}
$$

By virtue of (6), (7), (25), and the implicit function theorem [19], the domain $\mathscr{D}_{0}$ perturbs smoothly, for $\varepsilon \geqq 0$ small, to a simply connected domain $\mathscr{D}_{\varepsilon}$ such that

$$
a_{\varepsilon}>0 \quad \text { in } \mathscr{D}_{\varepsilon}, \quad a_{\varepsilon}<0 \quad \text { in } \mathbb{R}^{2} \backslash \overline{\mathscr{D}}_{\varepsilon}, \quad \frac{\partial a_{\varepsilon}}{\partial \nu_{\varepsilon}}<-c \quad \text { on } \partial \mathscr{D}_{\varepsilon},
$$

for some constant $c>0$, where $v_{\varepsilon}=v_{\varepsilon}(\mathbf{y})$ denotes the outward unit normal to $\partial \mathscr{D}_{\varepsilon}$. Let $\Gamma_{\varepsilon}$ be the simple, smooth closed curve defined by $\partial \mathscr{D}_{\varepsilon}$, and let $\ell_{\varepsilon}=\left|\Gamma_{\varepsilon}\right|$ denote its total length. Note that $\Gamma_{\varepsilon}$ is inside of an $\mathscr{O}\left(|\ln \varepsilon|^{\frac{1}{2}} \varepsilon\right)$-tubular neighborhood of $\partial \mathscr{D}_{0}$. We consider the natural parametrization $\gamma_{\varepsilon}=\gamma_{\varepsilon}(\theta)$ of $\Gamma_{\varepsilon}$ with positive orientation, where $\theta$ denotes an arc length parameter measured from a chosen point of $\Gamma_{\varepsilon}$. Slightly abusing notation, we let $\nu_{\varepsilon}(\theta)$ denote the outward unit normal to $\Gamma_{\varepsilon}$ [as in (33)]. Points $\mathbf{y}$ that are $\delta_{0}$-close to $\Gamma_{\varepsilon}$, for sufficiently small $\delta_{0}>0$ (independent of small $\varepsilon$ ), can be represented in the form

$$
\mathbf{y}=\gamma_{\varepsilon}(\theta)+t v_{\varepsilon}(\theta), \quad|t|<\delta_{0}, \theta \in\left[0, \ell_{\varepsilon}\right)
$$

where the map $\mathbf{y} \mapsto(t, \theta)$ is a local diffeomorphism (see [109, Sec. 14.6]). Note that $t<0$ in $\mathscr{D}_{\varepsilon}$. Abusing notation, frequently we will denote points $\mathbf{y}$ near $\Gamma_{\varepsilon}$ plainly by their image $(t, \theta)$ under the above mapping. From (32), (33), we have

$$
-\left(a_{\varepsilon}\right)_{t}(0, \theta)=W_{t}(0, \theta) \geqq c, \quad \theta \in\left[0, \ell_{\varepsilon}\right),
$$

for some constant $c>0$ and small $\varepsilon$. We define

$$
\beta_{\varepsilon}(\theta)=\left(-a_{t}(0, \theta)\right)^{\frac{1}{3}}>0, \quad \theta \in\left[0, \ell_{\varepsilon}\right) .
$$


It might be useful to point out that for the harmonic potential, described in (16), one can derive explicitly that

$$
\left[\beta_{\varepsilon}(\theta)\right]^{3}=2 \sqrt{\lambda_{\varepsilon}} \Lambda \sqrt{\Lambda^{-2} \cos ^{2}\left(\frac{2 \pi}{\ell_{\varepsilon}} \theta\right)+\sin ^{2}\left(\frac{2 \pi}{\ell_{\varepsilon}} \theta\right)}, \quad \theta \in\left[0, \ell_{\varepsilon}\right)
$$

We will also make use of the Hastings-McLeod solution [113] of the Painlevé-II equation [99], namely the unique solution $V$ of the boundary value problem:

$$
\begin{aligned}
& v_{x x}-v\left(v^{2}+x\right)=0, x \in \mathbb{R}, \\
& \quad v(x)-\sqrt{-x} \rightarrow 0, x \rightarrow-\infty ; \quad v(x) \rightarrow 0, x \rightarrow \infty .
\end{aligned}
$$

It is useful, in relation with (30), to point out here that $V_{x}<0, x \in \mathbb{R}$. The importance of the Hastings-Mcleod solution is that it will "lead" the minimizer $\eta_{\varepsilon}$ across $\partial \mathscr{D}_{0}$.

We can now state our main result:

Theorem 1. If $\varepsilon>0$ is sufficiently small, the unique real valued minimizer of $G_{\varepsilon}$ in $\mathscr{H}$ satisfies

$$
\eta_{\varepsilon}(\boldsymbol{y})=\varepsilon^{\frac{1}{3}} \beta_{\varepsilon}(\theta) V\left(\beta_{\varepsilon}(\theta) \frac{t}{\varepsilon^{\frac{2}{3}}}\right)+\mathscr{O}\left(\varepsilon+|t|^{\frac{3}{2}}\right)
$$

uniformly in $\left\{-d \leqq t \leqq 0, \theta \in\left[0, \ell_{\varepsilon}\right)\right\}$,

$$
\eta_{\varepsilon}(\boldsymbol{y})=\varepsilon^{\frac{1}{3}} \beta_{\varepsilon}(\theta) V\left(\beta_{\varepsilon}(\theta) \frac{t}{\varepsilon^{\frac{2}{3}}}\right)+\mathscr{O}\left(\varepsilon \mathrm{e}^{-c \varepsilon^{-\frac{2}{3}} t}\right)
$$

uniformly in $\left\{0 \leqq t \leqq d, \theta \in\left[0, \ell_{\varepsilon}\right)\right\}$, where $c, d>0$ are some small constants.

Given $D>0$, if $\varepsilon>0$ is sufficiently small, we have

$$
\eta_{\varepsilon}(\boldsymbol{y})-\sqrt{\lambda_{\varepsilon}-W(\boldsymbol{y})}=\mathscr{O}\left(\varepsilon^{2}|t|^{-\frac{5}{2}}\right)
$$

uniformly in $\left\{-d \leqq t \leqq-D \varepsilon^{\frac{2}{3}}, \theta \in\left[0, \ell_{\varepsilon}\right)\right\}$,

$$
\eta_{\varepsilon}(\boldsymbol{y})-\sqrt{\lambda_{\varepsilon}-W(\boldsymbol{y})}=\mathscr{O}\left(\varepsilon^{2}\right)
$$

uniformly in $\mathscr{D}_{\varepsilon} \backslash\left\{-d<t<0, \quad \theta \in\left[0, \ell_{\varepsilon}\right)\right\}$, and

$$
0<\eta_{\varepsilon}(\boldsymbol{y}) \leqq C \varepsilon^{\frac{1}{3}} \exp \left\{-c \varepsilon^{-\frac{2}{3}} \operatorname{dist}\left(\boldsymbol{y}, \partial \mathscr{D}_{0}\right)\right\}
$$

in $\mathbb{R}^{2} \backslash \mathscr{D}_{\varepsilon}$, for some constants $c, C>0$ independent of $\varepsilon$, where $\mathscr{O}(\cdot)$ denotes Landau's symbol (see Section 2 for the precise definition).

The potential of the associated linearized operator

$$
\boldsymbol{L}_{\varepsilon}(\varphi) \equiv \varepsilon^{2} \Delta \varphi-\left(3 \eta_{\varepsilon}^{2}(\boldsymbol{y})+W(\boldsymbol{y})-\lambda_{\varepsilon}\right) \varphi,
$$


satisfies

$$
3 \eta_{\varepsilon}^{2}(\boldsymbol{y})+W(\boldsymbol{y})-\lambda_{\varepsilon} \geqq \begin{cases}c \varepsilon^{\frac{2}{3}}+c|t|, & \text { if }|t| \leqq \delta \\ c+c|\boldsymbol{y}|^{p}, & \text { otherwise }\end{cases}
$$

The Lagrange multiplier $\varepsilon^{-2} \lambda_{\varepsilon}$ satisfies

$$
\lambda_{\varepsilon}-\lambda_{0}=\mathscr{O}\left(|\ln \varepsilon| \varepsilon^{2}\right)
$$

while the energy of $\eta_{\varepsilon}$ satisfies

$$
G_{\varepsilon}\left(\eta_{\varepsilon}\right)=\left(\frac{\lambda_{0}}{2}-\frac{1}{4} \int_{\mathbb{R}^{2}}\left(A^{+}\right)^{2} \mathrm{~d} \boldsymbol{y}\right) \varepsilon^{-2}+\frac{1}{12}\left(\int_{0}^{\ell_{0}} \beta_{0}^{3}(\theta) \mathrm{d} \theta\right)|\ln \varepsilon|+\mathscr{O}(1),
$$

as $\varepsilon \rightarrow 0$.

The main highlight of our result is that we rigorously prove that, close to $\partial \mathscr{D}_{0}$, the minimizer $\eta_{\varepsilon}$ behaves like (38). We emphasize that the rigorous derivation of the Pailevé-II equation from (8) was an unsettled open problem, see [9, pg. 13] (see also the discussion below on the recent paper [104]). In turn, as was noted in Section 8.1.3 of the book [9], this information is required as a stepping stone towards the open problem mentioned in Section 1.3 (see also Open Problem 8.1 in [10]), in order to obtain a lower bound for $\eta_{\varepsilon}$ in $\mathbb{R}^{2} \backslash \mathscr{D}_{0}$. In the current paper, under the additional assumption that $W_{t t}$ is strictly positive on $\partial \mathscr{D}_{0}$, we contribute towards this direction by obtaining a sharp lower bound in the strip-like domain of $\mathbb{R}^{2} \backslash \mathscr{D}_{0}$ described by $\operatorname{dist}\left(\mathbf{y}, \partial \mathscr{D}_{0}\right) \ll \varepsilon^{\frac{2}{5}}$ (see Remark 27 below).

We believe that our result opens the road for the rigorous description of the Painlevé region in recent experiments on three-dimensional Bose-Einstein condensates where a laser beam, modeled by a cylinder along the $z$ direction, is translated in the $x$ direction along the condensate (see [6,7]), and to understand the superfluid flow around an obstacle (see [9] and the references therein).

As we have already mentioned, our proof is based on perturbation arguments rather than variational ones or the method of upper and lower solutions (as was hoped for in [9, Sec. 8.3.1]). It relies mainly on the existence and asymptotic stability of the Hastings-McLeod solution (in the usual sense) in order to construct a solution $\mathbf{u}_{\varepsilon}$ of problem (8), "close" to $\sqrt{A^{+}}$, for small $\varepsilon>0$ (with the Lagrange multiplier $\lambda_{\varepsilon}$ treated as a known coefficient). Then, using the fact that the latter problem has a unique solution which follows from ideas of BREZIS and OswALD [43] (see Remark 16 below), namely $\eta_{\varepsilon}$, we infer that $\mathbf{u}_{\varepsilon} \equiv \eta_{\varepsilon}$. Actually, with the obvious modifications, an analogous result holds true for the minimizer of $G_{\varepsilon}$ in arbitrary dimensions. Furthermore, our method of proof can be adapted to treat the case where $G_{\varepsilon}(u)-\frac{\lambda}{2}\|u\|_{L^{2}\left(\mathbb{R}^{2}\right)}^{2}$ is minimized in $W_{0}^{1,2}(\mathbb{D})$, where $\mathbb{D}$ is a bounded annular domain such that $W=\lambda$ on the outer or inner part of its boundary, as in $[8,13]$, see Remark 17 below. For further generalizations we refer to Remarks 1, 2 below. 
It follows from the above estimates that the convergence of $\eta_{\varepsilon}$ to $\sqrt{A^{+}}$is the most regular possible (see Corollary 4 below). If we assume that $W_{t t} \geqq c>0$ on $\Gamma$, then we will show in Proposition 7 that bound (39) can be replaced by

$$
\eta_{\varepsilon}(\mathbf{y})=\varepsilon^{\frac{1}{3}} \beta_{\varepsilon}(\theta) V\left(\beta_{\varepsilon}(\theta) \frac{t}{\varepsilon^{\frac{2}{3}}}\right)\left[1+\mathscr{O}\left(\varepsilon^{\frac{2}{3}}\right)\left(\frac{t}{\varepsilon^{\frac{2}{3}}}\right)^{\frac{5}{2}}\right] .
$$

Estimate (45) improves (25), and was previously established in [125] in the special case of the model harmonic potential (16) by exploiting a scaling property of the corresponding Equation (8), see Remark 19 below, which is not available under our general assumptions. The above theorem has some other interesting consequences, which will be explored in Sections 4, 5: we can prove an analogous monotonicity property to (30) for $\eta_{\varepsilon}$ near $\partial \mathscr{D}_{0}$ without the simplifying assumption of radial symmetry on the potential $W$. We will see that estimates (28) and (29) are actually far from optimal. In addition, restricting ourselves to the radially symmetric case with $\mathscr{D}_{0}$ a ball, we can improve and sharpen the new estimates of [10] for the important auxiliary function $f_{\varepsilon}$, as described in (31). In fact, we believe that the estimates of Theorem 1 can be utilized in estimating the function $f_{\varepsilon}$ in the non-radial case, through Equation (194) below, which may ultimately lead to the resolution of the open problem raised in [10] (recall the discussion in Section 1.3).

To further illustrate the importance of our result, we emphasize that its method of proof can be adapted to produce similar results for semiclassical standing wave solutions of the defocusing NLS (21) , with $N \geqq 1$ and $q>2$, assuming that $W$ has the features described Section 1.1 with $\lambda$ in place of $\lambda_{0}$ and $\mathscr{D}_{0}$ a domain with $(N-1)$-dimensional boundary (see also Remark 31 below). (Recall that the minimizer $\eta_{\varepsilon}$ solves (8), and the discussion in the second part of Section 1.2.) We also emphasize that, when dealing directly with (22)_, our approach does not make any use of the techniques of BREZIS and OswALD [43], which were needed in previous approaches for establishing (26). In fact, our approach may produce sign changing solutions of (22)_, satisfying estimates analogous to those of Theorem 1 (see Remark 1 and Section 6 below).

A rigorous connection between semiclassical ground states of the defocusing nonlinear Schrödinger equation (21) _, in one space dimension, and solutions of the Painlevé-II equation (37) has been established recently in [190,191], for a related Hamiltonian system. We refer to the physical works [26,143,156,176] for formal expansions in one space dimension or radially symmetric cases, and to [205] for higher dimensions (see also [162]). In the case of the model harmonic potential $W(\mathbf{y})=|\mathbf{y}|^{2}, \mathbf{y} \in \mathbb{R}^{N}, N \geqq 1, \lambda=1$, and $q=3$, the problem of uniform asymptotic approximations of the ground state of the defocusing NLS (21) - using the Hastings-McLeod solution of the Painlevé-II equation, has been established on a rigorous level very recently in [104]. However, the approach of [104] relies crucially on the specific form of the model harmonic potential, which allows for a suitable global change of independent variables in Equation (22)_ (see Remark 21 below). The real delicacy of our result is not that it successfully connects $\eta_{\varepsilon}$ with $V$, but that we do so in a way that yields fine estimates, as can already be seen from (46), and the proof of (45) (see also the detailed estimates of [104] in the case of the 
model harmonic potential). The optimality of estimates (38), (40) is also suggested by Remark 14 below.

The analog of relation (44) may be used to study the spectrum of the linearization of the Gross-Pitaevskii equation (21) - at the corresponding standing wave solution (20), which is defined by the eigenvalue problem, in $L^{2}\left(\mathbb{R}^{N}\right) \times L^{2}\left(\mathbb{R}^{N}\right)$,

$$
\left\{\begin{array}{l}
-\varepsilon^{2} \Delta \phi+\left(W-\lambda+q\left|\eta_{\varepsilon}\right|^{q-1}\right) \phi=-\mu \psi \\
-\varepsilon^{2} \Delta \psi+\left(W-\lambda+\left|\eta_{\varepsilon}\right|^{q-1}\right) \psi=\mu \phi .
\end{array}\right.
$$

The above eigenvalue problem determines the spectral stability of the standing wave, with respect to the time evolution of the GP equation, and gives preliminary information for nonlinear analysis of orbital stability or more generally about the flow of $(21)_{-}$in a neighborhood of the standing wave (see $[28,106,110,164]$ ). More complex phenomena, such as those of pinned vortices (dark solitons) on top of the ground state [57,173], and the construction of traveling wave solutions with a stationary or traveling vortex ring to the GP equation [202,203], can also be understood from the analysis of (47). In particular, relation (44) plays an important role in the construction of excited states for (22)_ (see [174] and Section 6 herein). It seems that it was previously known only in the special case of the model harmonic potential (see [104]). In fact, even under the assumption of general radial symmetry, it does not follow from the recent estimates of [10].

Observe that the equation in (22), , with $q=3$, is equivalent, for $\varepsilon>0$, to

$$
\Delta u+(\lambda-W(\varepsilon y)) u-u^{3}, \quad y \in \mathbb{R}^{N},
$$

which when setting $\varepsilon=0$, and re-scaling appropriately (we assume that $\lambda>W(0)$ ), becomes the well known Allen-Cahn equation

$$
\Delta v-v\left(v^{2}-1\right)=0, \quad y \in \mathbb{R}^{N} .
$$

The above problem has received an enormous attention, see for instance [81] and the references therein. It seems plausible that our result can be combined with existing ones for the Allen-Cahn equation, and produce new interesting solutions for the Gross-Pitaevskii equation (22)_. We will elaborate more on this in Section 6 below.

Remark 1. Analogous assertions to those of Theorem 1 hold true if $\mathscr{D}_{0}$ is assumed to be the union of finitely many bounded smooth domains. In the latter case, one may construct sign changing solutions of (22) , whose absolute value converges uniformly to $\sqrt{A^{+}}$, as $\varepsilon \rightarrow 0$. The assumption that $\mathscr{D}_{0}$ is simply connected plays an important role only in Section 5 below. The degenerate case where some connected components of $\mathscr{D}_{0}$ "touch" is left as an open problem is Section 6.

Remark 2. With only minor modifications in the proof, all the assertions of Theorem 1 remain true if (4) is replaced by $\frac{1}{C} \leqq W(\mathbf{y})-\lambda_{0} \leqq C|\mathbf{y}|^{p},|\mathbf{y}| \geqq C$, for some $p>0$ (the second branch in (44) would have to be replaced by a constant). The fact that $p \geqq 2$ in (4) plays an important role only in Proposition 9 below. 
Remark 3. Let us keep in mind that $|t|=\operatorname{dist}\left(\mathbf{y}, \partial \mathscr{D}_{0}\right)+\mathscr{O}\left(|\ln \varepsilon|^{\frac{1}{2}} \varepsilon\right)$, wherever defined, and a-posteriori $|t|=\operatorname{dist}\left(\mathbf{y}, \partial \mathscr{D}_{0}\right)+\mathscr{O}\left(|\ln \varepsilon| \varepsilon^{2}\right)$, as $\varepsilon \rightarrow 0$ [recall (25), (45)].

Remark 4. An analogous result continuous to hold for the singularly perturbed elliptic equation

$$
\varepsilon^{2} \Delta u=F(u, \mathbf{y}),
$$

considered in the entire space $\mathbb{R}^{N}$ or in a bounded domain, where $F$ is such that the zero set of $F(u, \mathbf{y})=0$ undergoes a supercritical pitchfork bifurcation as the variable $\mathbf{y}$ crosses some surface (such conditions on $F$ can be found in [190]). In order to bring out clearly the underline ideas we refrain from any such generalization. In (39) the convergence is exponential because, in the case at hand, zero is a solution of $F=0$. However, there are no delay phenomena present, as $\mathbf{y}$ crosses $\partial \mathscr{D}_{0}$, in contrast to the first order equation $\varepsilon \dot{u}=F(u, t)$, as $t$ crosses the point corresponding to $\partial \mathscr{D}_{0}$ (see [33]).

\subsection{Outline of the Proof and Structure of the Paper}

The proof of Theorem 1 consists in showing that there exists a genuine solution of (8) "near" a suitably constructed smooth approximate solution, which in turn is "near" $\sqrt{A^{+}}$, provided the parameter $\varepsilon$ is small enough. We emphasize that we will consider the Lagrange multiplier $\varepsilon^{-2} \lambda_{\varepsilon}$, corresponding to the minimization of $G_{\varepsilon}$ in $\mathscr{H}$, as a known coefficient in (8) which, as we have already remarked, is known to satisfy (25). Then, by uniqueness (see also Remark 16 below), we will conclude that the obtained solution is actually the minimizer $\eta_{\varepsilon}$. Actually, we will prefer to work with the equivalent (for $\varepsilon>0$ ) problem in stretched variables $y=\varepsilon^{-\frac{2}{3}} \mathbf{y}$, see (51) below. The main steps of the proof are the following:

(i) Firstly, we construct a "good" smooth approximate solution for the (stretched) problem which we call $u_{\text {ap }}$. The function $u_{\text {ap }}$ is carefully built, along the lines set in [135], throughout Sections 3.1-3.3 in the following steps: Starting from the Hastings-McLeod solution $V$, described above, we construct an inner approximation $u_{\text {in }}$ that is valid only in a tubular neighborhood of the (stretched) curve $\varepsilon^{-\frac{2}{3}} \Gamma_{\varepsilon}$. Then, we obtain the desired (global) approximate solution $u_{\text {ap }}$ by patching $u_{\text {in }}$ with a subtle modification of the outer approximation $\sqrt{a_{\varepsilon}\left(\varepsilon^{\frac{2}{3}} y\right)^{+}}$. We emphasize that the use of this latter modification seems to be a key point in the whole construction. In fact, the matching of the inner approximation with the outer one is the major difficulty in the current problem, mainly due to the algebraic decay of $V$ to $\sqrt{-x}$ as $x \rightarrow-\infty$ [see (68) below]. For more details on this point, see the discussion in Section 3.2 and Remark 12 below. This difficulty was not present in [104], where the case of the model harmonic potential was considered, since in that case the special form of the equation allowed for the inner solution to be used globally, and thus no matching was necessary. 
(ii) Next, in Section 3.4, we study the linearized operator

$$
\mathscr{L}_{\varepsilon} \equiv \Delta-\varepsilon^{-\frac{2}{3}}\left(3 u_{\mathrm{ap}}^{2}-a\left(\varepsilon^{\frac{2}{3}} y\right)\right)
$$

about the approximate solution $u_{\text {ap }}$, and invert it in carefully chosen weighted spaces. We exploit a recent observation, due to Gallo and Pelinovsky [104, Lemma 2.2], which says that the potential $3 V^{2}+x$ of the linearization of (37) about the Hastings-McLeod solution is bounded below by some positive constant. In turn, we will show that this latter property implies that the same assertion holds true for the potential of $-\mathscr{L}_{\varepsilon}$, if $\varepsilon>0$ is small. In particular, for small $\varepsilon$, the operator $\mathscr{L}_{\varepsilon}$ is invertible. We point out that the previously mentioned lemma of [104] is of technical nature and can be bypassed at the expense of a more involved, but rather standard, analysis (see Remark 11 and Appendix A below). Our choice of weighted spaces, a variant of those considered in [171], is mainly motivated from the error term (88) below.

(iii) Finally, in Section 3.5, we look for a genuine solution of the stretched problem (51) in the form

$$
u_{\varepsilon}=u_{\text {ap }}+\varphi,
$$

where $\varphi$ is a correction. At this stage, we show that we can rephrase the problem as a fixed point problem for $\varphi$, which can easily be solved, if $\varepsilon>0$ is sufficiently small, using the fixed point theorem for contraction mappings. Then, in Section 3.6, taking advantage of the recent uniqueness result of [125] for problem (51) (see also Remark 16 below), we infer that the unique real valued minimizer of $G_{\varepsilon}$ in $\mathscr{H}$ satisfies $\eta_{\varepsilon}(\mathbf{y})=u_{\varepsilon}\left(\varepsilon^{-\frac{2}{3}} \mathbf{y}\right)$, $\mathbf{y} \in \mathbb{R}^{2}$. From this property, and the estimates derived from the construction of $u_{\varepsilon}$, we can deduce the validity of Theorem 1 . In particular, the proof of estimate (45) builds on (25), and uses the fact that $\left\|\eta_{\varepsilon}\right\|_{L^{2}\left(\mathbb{R}^{2}\right)}=1$.

The outline of the paper is the following. In Section 2 we will introduce notation and standard concepts that we will use throughout the paper. In Section 3 we will present the proof of our main result, as outlined above. In Section 4, as a byproduct of our construction, we will establish an analogous monotonicity property to (30) for the general case (with improvements), show that $\eta_{\varepsilon}$ has the maximal Hölder regularity available, improve bound (29), and generalize and considerably improve estimate (28). In Section 5, assuming that the potential $W$ is radially symmetric with $\mathscr{D}_{0}$ a ball, we will mainly rely on the results of Section 4 to refine and improve the recent estimates of [10] for the important auxiliary function $f_{\varepsilon}$ in (31). In Section 6 we will present some interesting open problems that are related to the current study. We will close the paper with two appendixes. In Appendix A we will reprove our main result concerning the linearized operator $\mathscr{L}_{\varepsilon}$ based on the asymptotic stability of the Hastings-McLeod solution (in the usual sense), rather than making use of the recent lemma of [104] which may not hold in other problems. Lastly, in Appendix B, we will provide a new more flexible and simple proof of the existence, and related properties, of the Hastings-McLeod solution through the study of problem (157) below. The results of the latter study seem to be new and to have interesting applications (see Remark 17 below). 


\section{Notation}

In the sequel, we will often suppress the obvious dependence on $\varepsilon$ of various functions and quantities. Furthermore by $c / C$ we will denote small/large generic constants, independent of $\varepsilon$, whose value will change from line to line. The value of $\varepsilon$ will constantly decrease so that all previous relations hold. The Landau symbol $\mathscr{O}(1), \varepsilon \rightarrow 0$, will denote quantities that remain uniformly bounded as $\varepsilon \rightarrow 0$, whereas $o(1)$ will denote quantities that approach zero as $\varepsilon \rightarrow 0$. By $C^{k, \alpha}$ or equivalently $C^{k+\alpha}, W^{k, p}, k, p \in \mathbb{Z} \cup\{\infty\}, \alpha \in[0,1)$, we will denote the usual Hölder, and Sobolev spaces respectively (see for instance [109]). Frequently, we will denote the minus case of (22) below plainly by $(22)_{-}$, etc.

We will identify the tubular neighborhood $B_{\delta}\left(\Gamma_{\varepsilon}\right)=\left\{\mathbf{y} \in \mathbb{R}^{2}: \operatorname{dist}\left(\mathbf{y}, \Gamma_{\varepsilon}\right)<\right.$ $\delta\}$, where $\delta<\delta_{0}$ [recall (34)], with $\Omega_{\delta}\left(\Gamma_{\varepsilon}\right)=\left\{|t|<\delta, \theta \in\left[0, \ell_{\varepsilon}\right)\right\}$, denoted simply by $\{|t|<\delta\}$, and $\mathbf{y}$ in $B_{\delta}\left(\Gamma_{\varepsilon}\right)$ by the corresponding pair $(t, \theta) \in \Omega_{\delta}\left(\Gamma_{\varepsilon}\right)$ as determined via (34).

\section{Proof of the Main Result}

\subsection{Setup Near the Curve}

In this subsection, suitably blowing up (8) around the curve $\Gamma_{\varepsilon}$, we will construct an inner approximation which is valid only near the curve, for small $\varepsilon>0$, whose profile normal to the curve will be that of a (scaled) Hastings-McLeod solution (see also [205]). To this end, we will follow the general lines set in [78] which dealt with the focusing case $(22)_{+}$.

Formally neglecting the term $\varepsilon^{2} \Delta u$ in (8), we get the outer approximation $\sqrt{a_{\varepsilon}(\mathbf{y})^{+}}$. However, the Laplacian of the latter is not even bounded on the boundary of $\mathscr{D}_{\varepsilon}$. Hence, the outer approximation fails in the vicinity of $\partial \mathscr{D}_{0}$. An inner approximation is thus needed, playing the role of a "bridge" as $\mathbf{y}$ crosses that boundary.

In the coordinates $(t, \theta)$, near $\Gamma_{\varepsilon}$, the metric can be parameterized as

$$
g_{t, \theta}=\mathrm{d} t^{2}+(1+k t)^{2} \mathrm{~d} \theta^{2},
$$

and the Laplacian operator becomes

$$
\Delta_{t, \theta}=\frac{\partial^{2}}{\partial t^{2}}+\frac{1}{(1+k t)^{2}} \frac{\partial^{2}}{\partial \theta^{2}}+\frac{k}{1+k t} \frac{\partial}{\partial t}-\frac{k^{\prime} t}{(1+k t)^{3}} \frac{\partial}{\partial \theta},
$$

where $k_{\varepsilon}(\theta)$ is the curvature of $\Gamma_{\varepsilon}$ (see for instance $[17,95]$ ). Note that $k_{\varepsilon}$ and its derivatives depend smoothly on $\varepsilon \geqq 0$.

Remark 5. $\left(t, \gamma_{\varepsilon}(\theta)\right)$ are known in the literature as Fermi coordinates, see for instance [140], and are frequently employed in the study of perturbation problems involving solutions concentrating on manifolds (see for example [78]). Interestingly enough, they owe their name to the physicist E. Fermi in the title of the current paper! 
In stretched variables

$$
y=\varepsilon^{-\frac{2}{3}} \mathbf{y}
$$

problem (8) becomes

$$
\Delta u-\varepsilon^{-\frac{2}{3}} u\left(u^{2}-a_{\varepsilon}\left(\varepsilon^{\frac{2}{3}} y\right)\right)=0, \quad u>0 \quad \text { in } \mathbb{R}^{2}, \quad \lim _{|y| \rightarrow \infty} u(y)=0,
$$

where $a_{\varepsilon}$ was defined in (32).

In the sequel we will denote

$$
\tilde{\mathscr{D}}_{\varepsilon}=\varepsilon^{-\frac{2}{3}} \mathscr{D}_{\varepsilon} \quad \text { and } \quad \tilde{\Gamma}_{\varepsilon}=\varepsilon^{-\frac{2}{3}} \Gamma_{\varepsilon} .
$$

Let

$$
(s, z)=\varepsilon^{-\frac{2}{3}}(t, \theta)
$$

be natural stretched coordinates associated to the curve $\tilde{\Gamma}_{\varepsilon}$, now defined for

$$
s \in\left(-\delta_{0} \varepsilon^{-\frac{2}{3}}, \delta_{0} \varepsilon^{-\frac{2}{3}}\right), \quad z \in\left[0, \varepsilon^{-\frac{2}{3}} \ell_{\varepsilon}\right) .
$$

In the coordinates $(s, z)$, near $\tilde{\Gamma}_{\varepsilon}$, the metric can be written as

$$
g_{s, z}=\mathrm{d} s^{2}+\left(1+\varepsilon^{\frac{2}{3}} k s\right)^{2} \mathrm{~d} z^{2},
$$

and the Laplacian for $u$ expressed in these coordinates becomes

$$
\Delta u=u_{z z}+u_{s s}+B_{1}(u)
$$

where

$$
\begin{aligned}
B_{1}(u)= & -\left[1-\frac{1}{\left(1+\varepsilon^{\frac{2}{3}} k\left(\varepsilon^{\frac{2}{3}} z\right) s\right)^{2}}\right] u_{z z}+\frac{\varepsilon^{\frac{2}{3}} k\left(\varepsilon^{\frac{2}{3}} z\right)}{1+\varepsilon^{\frac{2}{3}} k\left(\varepsilon^{\frac{2}{3}} z\right) s} u_{s} \\
& -\frac{\varepsilon^{\frac{4}{3}} s k^{\prime}\left(\varepsilon^{\frac{2}{3}} z\right)}{\left(1+\varepsilon^{\frac{2}{3}} k\left(\varepsilon^{\frac{2}{3}} z\right) s\right)^{3}} u_{z} .
\end{aligned}
$$

Hence, Equation (51) takes the form

$$
R(u) \equiv u_{z z}+u_{s s}+B_{1}(u)-\varepsilon^{-\frac{2}{3}} u\left(u^{2}-a\left(\varepsilon^{\frac{2}{3}} s, \varepsilon^{\frac{2}{3}} z\right)\right)=0,
$$

in the region (54). Observe that all terms in the operator $B_{1}$ have $\varepsilon^{\frac{2}{3}}$ as a common factor, more precisely we can write

$$
B_{1}(u)=\varepsilon^{\frac{2}{3}} a_{0}\left(\varepsilon^{\frac{2}{3}} s, \varepsilon^{\frac{2}{3}} z\right) u_{s}+\varepsilon^{\frac{4}{3}} s a_{1}\left(\varepsilon^{\frac{2}{3}} s, \varepsilon^{\frac{2}{3}} z\right) u_{z}+\varepsilon^{\frac{2}{3}} s a_{2}\left(\varepsilon^{\frac{2}{3}} s, \varepsilon^{\frac{2}{3}} z\right) u_{z z},
$$

for certain smooth functions $a_{j}(t, \theta), j=0,1,2$. 
We now consider a further change of variables in Equation (58) with the property that it (roughly) replaces at main order the function $a$ by one that has constant gradient along $\tilde{\Gamma}_{\varepsilon}$. Let $\beta$ be as in (36), then we define $v(x, z)$ by the relation

$$
\left\{\begin{array}{l}
u(s, z)=\varepsilon^{\frac{1}{3}} \beta\left(\varepsilon^{\frac{2}{3}} z\right) v(x, z), \\
x=\beta\left(\varepsilon^{\frac{2}{3}} z\right) s .
\end{array}\right.
$$

Choosing a smaller $\delta_{0}$, if necessary, we may assume that the coordinates $(x, z)$ are also defined for $|x| \leqq \delta_{0} \varepsilon^{-\frac{2}{3}}, z \in\left[0, \varepsilon^{-\frac{2}{3}} \ell_{\varepsilon}\right)$. We want to express Equation (58) in terms of these new coordinates. We compute:

$$
\left\{\begin{aligned}
u_{s}= & \varepsilon^{\frac{1}{3}} \beta^{2} v_{x}, \\
u_{s s}= & \varepsilon^{\frac{1}{3}} \beta^{3} v_{x x}, \\
u_{z}= & \varepsilon \beta^{\prime} v+\varepsilon^{\frac{1}{3}} \beta v_{z}+\varepsilon \beta^{\prime} x v_{x}, \\
u_{z z}= & \varepsilon^{\frac{5}{3}} \beta^{\prime \prime} v+2 \varepsilon^{\frac{5}{3}}\left(\beta^{\prime}\right)^{2} \beta^{-1} x v_{x}+2 \varepsilon \beta^{\prime} v_{z}+2 \varepsilon \beta^{\prime} x v_{x z}+\varepsilon^{\frac{1}{3}} \beta v_{z z} \\
& +\varepsilon^{\frac{5}{3}} \beta^{\prime \prime} x v_{x}+\varepsilon^{\frac{5}{3}}\left(\beta^{\prime}\right)^{2} \beta^{-1} x^{2} v_{x x} .
\end{aligned}\right.
$$

In order to write down the equation, it is also convenient to expand

$$
\begin{aligned}
a\left(\varepsilon^{\frac{2}{3}} s, \varepsilon^{\frac{2}{3}} z\right) & =a_{t}\left(0, \varepsilon^{\frac{2}{3}} z\right) \varepsilon^{\frac{2}{3}} s+a_{3}\left(\varepsilon^{\frac{2}{3}} s, \varepsilon^{\frac{2}{3}} z\right) \varepsilon^{\frac{4}{3}} s^{2} \\
& \stackrel{(36)}{=}-\beta^{2} \varepsilon^{\frac{2}{3}} x+a_{3}\left(\varepsilon^{\frac{2}{3}} s, \varepsilon^{\frac{2}{3}} z\right) \varepsilon^{\frac{4}{3}} s^{2},
\end{aligned}
$$

for some bounded function $a_{3}(t, \theta)$. It turns out that $u$ solves (58) if and only if $v$, defined by (60), solves

$$
S(v) \equiv \varepsilon^{-\frac{1}{3}} \beta^{-3} R(u)=v_{x x}-v\left(v^{2}+x\right)+B_{2}(v)=0,
$$

where $B_{2}(v)$ is a differential operator defined by

$$
B_{2}(v)=\varepsilon^{\frac{2}{3}} \tilde{a}_{3} x^{2} v+\varepsilon^{-\frac{1}{3}} \beta^{-3} u_{z z}+\varepsilon^{-\frac{1}{3}} \beta^{-3} B_{1}(u),
$$

where the bounded function $\tilde{a}_{3}(t, \theta)$ is evaluated at $\left(\varepsilon^{\frac{2}{3}} \beta^{-1} x, \varepsilon^{\frac{2}{3}} z\right)$, and $B_{1}$ is the differential operator in (57) where derivatives are expressed in terms of formulas (61) and $s$ replaced by $\beta^{-1} x$. (Note that $B_{2}(v)=\beta^{-2} v_{z z}+\tilde{B}_{2}(v)$, where all the terms in the operator $\tilde{B}_{2}$ carry $\varepsilon^{\frac{2}{3}}$ in front of them.)

Our first criterion for choosing an approximate solution $v$ of (63) is that $S(v)$ is small. In view of (63), (64), it is natural to choose a $v$ that depends only on $x$, and solves the second-order non-autonomous ordinary differential equation:

$$
v_{x x}-v\left(v^{2}+x\right)=0, \quad x \in \mathbb{R}
$$

which is known as the Painlevé-II equation, a particular case of the second Painlevé transcendent [99]. Then, keeping in mind that the inner profile $\varepsilon^{\frac{1}{3}} \beta v(\beta s)$ of (51) should match [198, Chpt. 5] with the outer profile $\sqrt{a\left(\varepsilon^{\frac{2}{3}} y\right)^{+}}$, as $s \rightarrow \pm \infty$, it is easy to see that the appropriate asymptotic behavior of $v$ should be

$$
v(x)-\sqrt{-x} \rightarrow 0, \quad x \rightarrow-\infty ; \quad v(x) \rightarrow 0, \quad x \rightarrow \infty .
$$


(Observe that the $s$ variable is defined in $\left(-\delta_{0} \varepsilon^{-\frac{2}{3}}, \delta_{0} \varepsilon^{-\frac{2}{3}}\right)$, but the natural domain for this variable is the infinite line.) More precisely, recalling (62), we get that

$$
\sqrt{a_{\varepsilon}\left(\varepsilon^{\frac{2}{3}} y\right)^{+}}=\varepsilon^{\frac{1}{3}} \beta \sqrt{-\beta s}+\mathscr{O}\left(\varepsilon|s|^{\frac{3}{2}}\right)
$$

if $-\delta_{0} \varepsilon^{-\frac{2}{3}} \leqq s \leqq 0$, as $\varepsilon \rightarrow 0$, while on the other side it holds that $a_{\varepsilon}\left(\varepsilon^{\frac{2}{3}} y\right)=0$ if $0 \leqq s \leqq \delta_{0} \varepsilon^{-\frac{2}{3}}$.

The following proposition holds:

Proposition 1. Problem (65)-(66) has a unique solution $V$, called Hastings-McLeod solution. Furthermore, we have

$$
V_{x}<0 \text { in } \mathbb{R} \text {. }
$$

The following estimates hold:

$$
\begin{gathered}
-C|x|^{-\frac{5}{2}}<V(x)-\sqrt{-x}<0, x \rightarrow-\infty ; \\
0<V(x)<C x^{-\frac{1}{4}} \mathrm{e}^{-\frac{2}{3} x^{\frac{3}{2}}}, x \geqq 1,
\end{gathered}
$$

for some constant $C>0$, and

$$
\left\{\begin{array}{lll}
V_{x}=-\frac{1}{2}(-x)^{-\frac{1}{2}}+\mathscr{O}\left(|x|^{-\frac{7}{2}}\right), & V_{x x}=-\frac{1}{4}(-x)^{-\frac{3}{2}}+\mathscr{O}\left(|x|^{-\frac{9}{2}}\right), & x \rightarrow-\infty \\
V_{x} V=-\frac{1}{2}+\mathscr{O}\left(|x|^{-3}\right), & V^{2}+x=\mathscr{O}\left(|x|^{-2}\right), & x \rightarrow-\infty \\
\left|V_{x}\right|+\left|V_{x x}\right| \leqq C \mathrm{e}^{-c x^{\frac{3}{2}}}, & x>0 .
\end{array}\right.
$$

The potential of the associated linearized operator

$$
\mathscr{M}(\varphi) \equiv \varphi_{x x}-\left(3 V^{2}(x)+x\right) \varphi
$$

satisfies

$$
3 V^{2}(x)+x \geqq c>0, \quad x \in \mathbb{R} .
$$

Proof. We will provide a sketch of proof, underlining the main ideas, and refer the interested reader to the original works.

The existence and uniqueness of a solution $V$ of (65)-(66) have both been shown by HASTINGS and MCLEOD [113,116] using a "shooting" technique (they also mention an unpublished proof of Conley). Recently, a new proof of existence has been given in [18] by the method of upper and lower solutions. Motivated from some problems that we mentioned in Section 1.2 (see also Remark 17 below), we will provide in Appendix B a new different proof of existence and uniqueness which seems to be simpler and more flexible. Another different and rather short proof has been given very recently in [202, Lemma 2.4]. Relation (67) has also been shown in the aforementioned references, and in Appendix B herein. We note that, by looking at the equation for $Z \equiv(-x)^{-\frac{1}{2}} V$, one can show that $V$ has a unique inflection point (see [113]); notice also that $V-\sqrt{-x}$ is convex whenever nonnegative. 
The asymptotic behavior of $V$, as $x \rightarrow \pm \infty$, is described in great detail in Theorem 11.7 of [99] (see also [129]). In particular, it follows that relations (68)(69) hold true. Let us provide some insight into these, at first sight, complex formulae. The first relation of (68) can be formally derived as follows: assume that $V-\sqrt{-x} \sim \alpha(-x)^{-\beta}$ as $x \rightarrow-\infty$, for some $\alpha \in \mathbb{R}, \beta>0$; plugging this ansatz in (65), keeping in mind that $V+\sqrt{-x} \sim 2 \sqrt{-x}$ and (the expectation) that $V_{x x} \sim-\frac{1}{4}(-x)^{-\frac{3}{2}}$ as $x \rightarrow-\infty$, we readily find that $\alpha=-\frac{1}{8}, \beta=\frac{5}{2}$ (see also [176, pg. 160]). A rigorous derivation can be given by simply writing down the equation for the function $V-\sqrt{-x}$ (or better yet for $V-\sqrt{-x}+\frac{1}{8}(-x)^{-\frac{5}{2}}$ ), and then applying in the resulting identity the following simple lemma, which can be proven by a standard barrier argument:

Lemma (cf. [104, Lemma 2.1] or [134, Lemma 3.10]) Suppose that $\phi \in C^{2}, q \in C$ satisfy

$$
-\phi^{\prime \prime}+q(x) \phi=\mathscr{O}\left(|x|^{-\alpha}\right),
$$

$\phi \rightarrow 0$, and $q(x) \geqq c|x|$, as $x \rightarrow-\infty$, for some constants $\alpha, c>0$. Then we have

$$
\phi=\mathscr{O}\left(|x|^{-\alpha-1}\right) \text { as } x \rightarrow-\infty .
$$

In passing, we note that the above lemma can also be proven by rewriting the equation in terms of the new independent variable $\xi=-(-x)^{\frac{3}{2}}$ and then applying a lemma of Hérve and Hérve [120, pg. 435], see also [117, Prop. 3.1]. On the other side, because of the second condition in (66), the nonlinear term in (65) can be (formally) neglected for $x \rightarrow \infty$, yielding Airy's equation [32, pg. 100], namely

$$
y^{\prime \prime}=x y,
$$

predicting that

$$
V(x) \sim \gamma \operatorname{Ai}(x) \text { as } x \rightarrow \infty,
$$

for some constant $\gamma>0$, where $\operatorname{Ai}(\cdot)$ denotes the standard Airy's function [see also (74) below]. For future reference, we recall from [32] that the two independent solutions of Airy's equation can be taken to have the asymptotic behavior

$$
\operatorname{Ai}(x) \sim \frac{1}{2} \pi^{-\frac{1}{2}} x^{-1 / 4} \mathrm{e}^{-2 x^{3 / 2} / 3} \text { and } \operatorname{Bi}(x) \sim \pi^{\frac{1}{2}} x^{-1 / 4} \mathrm{e}^{2 x^{3 / 2} / 3}, x \rightarrow \infty,
$$

see also the discussion leading to formulae (B.250) below. Relation (73), which clearly implies the validity of the second estimate in (68), can be established rigorously directly from the variation of constants formula

$$
V(x)=\gamma \operatorname{Ai}(x)+2 \int_{x}^{\infty}\{\operatorname{Ai}(x) \operatorname{Bi}(t)-\operatorname{Bi}(x) \operatorname{Ai}(t)\} V^{3}(t) \mathrm{d} t,
$$

see also [16, Lemma B.1]. It is worthwhile to note that the exact value of $\gamma$ was determined to be

$$
\gamma=1
$$


in [113], using an integral equation derived by inverse scattering techniques of Ablowitz and Segur. An implication of this beautiful formula, for the physics of Bose-Einstein condensation, has been pointed out in [161]. The estimates in (69) can be established in a similar manner. For example, the asymptotic behavior of $V_{x}$ as $x \rightarrow-\infty$ follows by writing down the equation for the function $V_{x}+\frac{1}{2}(-x)^{-\frac{1}{2}}$ and then applying the above lemma.

Finally, the lower bound (71) has been proven recently in [104, Lemma 2.2]; despite of its simple appearance, its proof takes almost three pages! Actually, we were surprised to find that such an apparently simple relation proves to be so recalcitrant. We emphasize that, for the purposes of the current paper, it can be bypassed (something that we did not do) at the expense of some extra work (see Remark 11 below). On the other side, it is quite painless to show that the operator $\mathscr{M}$ still satisfies the maximum principle without knowledge of the latter lemma. In other words:

$$
\begin{aligned}
& \text { "whenever } w \in C^{2}(\mathbb{R}), \mathscr{M}(w) \leqq 0, \quad \text { and } \liminf _{|x| \rightarrow \infty} w(x) \geqq 0, \\
& \text { then necessarily } w \geqq 0 " .
\end{aligned}
$$

For this, recall that the typical way towards establishing the maximum principle for an elliptic operator $L$ in an open set $\Omega$ is to first show that

"there exists a positive upper-solution $\phi$ of $L(\phi)=0$ in $\Omega$ ",

see [52] for more details. If this holds, then adding one of various additional assumptions on $\phi$ (the simplest one being $\phi \geqq c$ with $c$ a positive constant), it does guarantee the maximum principle to hold, see [35]. Indeed, in bounded domains, (78) is a necessary and almost sufficient condition for the maximum principle to hold, see Corollary 2.1 of [35]. However, in unbounded domains one has to be more careful to deal with infinity. Having this in mind, firstly note that

$$
\mathscr{M}\left(-V_{x}\right)=-V<0 ; \quad \mathscr{M}(V)=-2 V^{3}<0,
$$

and recall that $-V_{x}>0, V>0$ in $\mathbb{R}$. So, condition (78), with $L=-\mathscr{M}$, is satisfied by either one of $-V_{x}$ or $V$. Nevertheless, observe that one faces a difficulty when proceeding as in [35], namely applying the standard maximum principle in the equation satisfied by $\frac{w}{-V_{x}}$ or $\frac{w}{V}$ [where $w$ is as in (77)]. This is because $-V_{x}$ and $V$ vanish as $x \rightarrow \pm \infty$ and $x \rightarrow \infty$ respectively, making the signs of $\lim \inf _{x \rightarrow \pm \infty} \frac{w}{-V_{x}}$ and $\liminf _{x \rightarrow \infty} \frac{w}{V}$ unclear (in the case where one of $\lim \inf _{x \rightarrow \pm \infty} w$ equals zero). (This subtle point seems to have been overlooked in [202].) Instead, we consider the function

$$
\varphi_{\epsilon}=\frac{w-\epsilon V_{x}}{V}, \quad \epsilon>0,
$$

noting that, thanks to (72)-(74) [see also (182) below], we have $\lim _{x \rightarrow-\infty} \varphi_{\epsilon} \geqq 0$ and $\lim _{x \rightarrow \infty} \varphi_{\epsilon}=\infty$. Using (77), (79), and applying the standard maximum principle in the equation satisfied by $\varphi_{\epsilon}$, we obtain that $\varphi_{\epsilon}>0 \mathrm{in} \mathbb{R}$, for every $\epsilon>0$. Then, letting $\epsilon \rightarrow 0$ we infer that $w \geqq 0$ in $\mathbb{R}$ which is the desired assertion in (77).

The sketch of the proof of the proposition is complete. 
We can now define the inner solution of problem (51), in the neighborhood of $\tilde{\Gamma}_{\varepsilon}$ described by $\left\{|x| \leqq \delta_{0} \varepsilon^{-\frac{2}{3}}, \quad z \in\left[0, \varepsilon^{-\frac{2}{3}} \ell_{\varepsilon}\right)\right\}$, as

$$
u_{\text {in }}(y)=\varepsilon^{\frac{1}{3}} \beta\left(\varepsilon^{\frac{2}{3}} z\right) V(x),
$$

[recall (60)].

Let $L>0$ be a large constant to be determined independently of small $\varepsilon>0$. We consider the neighborhood of $\tilde{\Gamma}_{\varepsilon}$ described by

$$
U_{\varepsilon}=\left\{-2 L \leqq x \leqq \delta_{0} \varepsilon^{-\frac{2}{3}}, \quad z \in\left[0, \varepsilon^{-\frac{2}{3}} \ell_{\varepsilon}\right)\right\} .
$$

The following proposition contains the main estimate regarding $u_{\text {in }}$.

Proposition 2. If $\varepsilon$ is sufficiently small, the inner approximation $u_{\mathrm{in}}$, defined in (80), satisfies

$$
\left\|\Delta u_{\text {in }}-\varepsilon^{-\frac{2}{3}} u_{\text {in }}\left(u_{\text {in }}^{2}-a\left(\varepsilon^{\frac{2}{3}} y\right)\right)\right\|_{L^{\infty}\left(U_{\varepsilon}\right)} \leqq C \varepsilon .
$$

Proof. From the calculation leading to (63), and (64), we find that

$$
\begin{aligned}
\left|\Delta u_{\text {in }}-\varepsilon^{-\frac{2}{3}} u_{\text {in }}\left(u_{\text {in }}^{2}-a\left(\varepsilon^{\frac{2}{3}} y\right)\right)\right| & =\left|R\left(u_{\text {in }}\right)\right|=\varepsilon^{\frac{1}{3}} \beta^{3}|S(V)| \\
& \leqq C \varepsilon\left[\left(x^{2}+\varepsilon^{\frac{2}{3}}\right) V+\left|V_{x}\right|+|x|\left|V_{x x}\right|\right]
\end{aligned}
$$

pointwise in $U_{\varepsilon}$. The desired estimate (82) now follows at once from the above relation, via (68), (69) and (81).

The proof of the proposition is complete.

Remark 6. From the geometric singular perturbation viewpoint [190], the HastingsMcLeod solution corresponds to a special trajectory of the (de-singularized) blownup system that connects two equilibria on a sphere. The fact that this connection is transverse, which allows for a perturbation argument, follows from the nondegeneracy of the Hastings-McLeod solution (as defined in Remark 11 below). In this regard, see also Remark 9 below.

Remark 7. In the context of singular perturbation problems, the Hastings-McLeod solution first served as a basis for an inner solution in plasma physics (see [76]). Since then, it has been (formally) used to describe layered structures in problems involving crystalline interphase boundaries $[18,190,191]$, patterns of convection in rectangular platform containers [75], self-similar parabolic optical solitary waves [41], and the Navier-Stokes and continuity equations for axisymmetric flow [157].

Remark 8. In well known singularly perturbed elliptic problems, such as the focusing NLS (recall the discussion in Section 1.2.2) or the spatially inhomogeneous Allen-Cahn equation (see for instance $[86,151,201]$ ), the corresponding inner profile is determined by special solutions of autonomous second order elliptic equations (posed in less or equal dimensions). In the former case the corresponding equation is

$$
\Delta u-u+|u|^{q-1} u=0
$$


while in the latter it is Equation (49). In contrast, in the problem at hand the corresponding Equation (65) is non-autonomous, as was the case in [47,71,73,134], and [135]. The interested reader can verify that similar situations also occur in the singularly perturbed Fisher's equation [118, Chpt. 10, Exc.3] (see also [165]), and in the spatially inhomogeneous Allen-Cahn equation that we mentioned previously, treated in $[86,151]$, if the spatial inhomogeneity is not strictly positive but vanishes at certain points (or submanifolds) of the domain (have in mind the first part of Section 1.2.3).

\subsection{Set Up Away from the Curve}

In this subsection, adapting an idea of [134] (see also [135]), we suitably perturb, in $\tilde{\mathscr{D}}_{\varepsilon}$, the outer approximation

$$
u_{\text {out }} \equiv \sqrt{a\left(\varepsilon^{\frac{2}{3}} y\right)^{+}}
$$

to an improved outer approximation $\tilde{u}_{\text {out }}$, which is closer to the inner approximation $u_{\text {in }}$ near the curve $\tilde{\Gamma}_{\varepsilon}$, for small $\varepsilon>0$. We emphasize that this is a key step in our construction of an approximate solution for (51). Our underlying plan, carried out in the following subsection, is to smoothly interpolate between $\tilde{u}_{\text {out }}$ and $u_{\text {in }}$, and $u_{\text {in }}$ and zero (in $\mathbb{R}^{2} \backslash \tilde{\mathscr{D}}_{\varepsilon}$ ), near the curve $\tilde{\Gamma}_{\varepsilon}$, in order to obtain an approximate solution $u_{\text {ap }}$ of (51) that is valid in all of $\mathbb{R}^{2}$. Interpolating directly between $u_{\text {out }}$, as defined in (84), and $u_{\text {in }}$ in $\tilde{\mathscr{D}}_{\varepsilon}$ is not standard due to the following obstructions: The inner approximation $u_{\text {in }}$ leaves a remainder in (51) that grows with respect to the (negative) distance from $\tilde{\Gamma}_{\varepsilon}$, as can be seen from (68), (83); $V$ converges algebraically slowly to $\sqrt{-x}$ as $x \rightarrow-\infty$, recall (68). (For more details on this subtle point we refer to Remark 12 below.) In the one-dimensional case, an elegant solution to this can be given by geometric singular perturbation theory, see [190] and Remark 9 below. In contrast, thanks to the (super-) exponentially fast convergence of $V$ to zero, as $x \rightarrow \infty$, interpolating directly between $u_{\text {in }}$ and zero in $\mathbb{R}^{2} \backslash \tilde{\mathscr{D}}_{\varepsilon}$ is rather standard. (This last situation occurs in the construction of spike-layered solutions of the focusing $(22)_{+}$, see $[20,78,98]$, and transition-layered solutions of Allen-Cahn type equations, see [79,96].)

Let $\delta<\frac{\delta_{0}}{100(1+\max \beta)}$ be a fixed number. We consider a smooth cutoff function

$$
n_{\delta}(t)= \begin{cases}1, & |t| \leqq \delta \\ 0, & |t| \geqq 2 \delta\end{cases}
$$

Denote as well

$$
\chi_{\delta}(x)=n_{\delta}\left(\varepsilon^{\frac{2}{3}} x\right),
$$

where $x$ is the coordinate in (60).

We define our outer approximation in the domain $\tilde{\mathscr{D}}_{\varepsilon} \backslash\{-L<\beta s<0, z \in$ $\left.\left[0, \varepsilon^{-\frac{2}{3}} \ell_{\varepsilon}\right)\right\}$ to be

$$
\tilde{u}_{\text {out }}(y) \equiv\left\{a\left(\varepsilon^{\frac{2}{3}} y\right)+\varepsilon^{\frac{2}{3}} \beta^{2} \chi_{\delta}(\beta s)\left[\beta s+V^{2}(\beta s)\right]\right\}^{\frac{1}{2}} .
$$


Note that, thanks to (33), (69), if $L$ is sufficiently large then $\tilde{u}_{\text {out }}$ is well defined for small $\varepsilon$.

The following proposition makes delicate use of estimates (68), (69), and contains the main properties of $\tilde{u}_{\text {out }}$. In some sense, it is the "heart" of the present paper.

Proposition 3. We have

$$
\Delta \tilde{u}_{\text {out }}-\varepsilon^{-\frac{2}{3}} \tilde{u}_{\text {out }}\left(\tilde{u}_{\text {out }}^{2}-a\left(\varepsilon^{\frac{2}{3}} y\right)\right)=\mathscr{O}\left(\varepsilon|s|^{-\frac{1}{2}}\right)
$$

uniformly in $\left\{-\delta \varepsilon^{-\frac{2}{3}} \leqq \beta s \leqq-L, z \in\left[0, \varepsilon^{-\frac{2}{3}} \ell_{\varepsilon}\right)\right\}$, and

$$
\Delta \tilde{u}_{\text {out }}-\varepsilon^{-\frac{2}{3}} \tilde{u}_{\text {out }}\left(\tilde{u}_{\text {out }}^{2}-a\left(\varepsilon^{\frac{2}{3}} y\right)\right)=\mathscr{O}\left(\varepsilon^{\frac{4}{3}}\right)
$$

uniformly in $\tilde{\mathscr{D}}_{\varepsilon} \backslash\left\{-\delta \varepsilon^{-\frac{2}{3}} \leqq \beta s<0, z \in\left[0, \varepsilon^{-\frac{2}{3}} \ell_{\varepsilon}\right)\right\}$, as $\varepsilon \rightarrow 0$. Moreover, if $\varepsilon>0$ is sufficiently small, we have

$$
\left|\tilde{u}_{\text {out }}-u_{\text {in }}\right| \leqq C \varepsilon|s|^{\frac{3}{2}}, \quad\left|\nabla\left(\tilde{u}_{\text {out }}-u_{\text {in }}\right)\right| \leqq C \varepsilon|s|^{\frac{1}{2}}, \quad\left|\Delta\left(\tilde{u}_{\text {out }}-u_{\text {in }}\right)\right| \leqq C \varepsilon|s|^{-\frac{1}{2}}
$$

in $\left\{-\delta \varepsilon^{-\frac{2}{3}} \leqq \beta s \leqq-L, z \in\left[0, \varepsilon^{-\frac{2}{3}} \ell_{\varepsilon}\right)\right\}$, and

$$
\left|\tilde{u}_{\text {out }}-\sqrt{a\left(\varepsilon^{\frac{2}{3}} y\right)}\right| \leqq C \varepsilon^{\frac{1}{3}}|s|^{-\frac{5}{2}}
$$

in $\left\{-2 \delta \varepsilon^{-\frac{2}{3}} \leqq \beta s \leqq-L, z \in\left[0, \varepsilon^{-\frac{2}{3}} \ell_{\varepsilon}\right)\right\}$.

Proof. In $\left\{-\delta \varepsilon^{-\frac{2}{3}} \leqq \beta s \leqq-L, z \in\left[0, \varepsilon^{-\frac{2}{3}} \ell_{\varepsilon}\right)\right\}$, we have $\chi_{\delta}=1$ and we can compute that

$$
\begin{aligned}
-\varepsilon^{-\frac{2}{3}} \tilde{u}_{\text {out }}\left(\tilde{u}_{\text {out }}^{2}-a\left(\varepsilon^{\frac{2}{3}} y\right)\right) & =-\beta^{2}\left[a\left(\varepsilon^{\frac{2}{3}} y\right)+\varepsilon^{\frac{2}{3}} \beta^{3} s+\varepsilon^{\frac{2}{3}} \beta^{2} V^{2}\right]^{\frac{1}{2}}\left[\beta s+V^{2}(\beta s)\right] \\
\operatorname{via}(33),(36) & =-\beta^{2}\left[\mathscr{O}\left(\varepsilon^{\frac{4}{3}} s^{2}\right)+\varepsilon^{\frac{2}{3}} \beta^{2} V^{2}\right]^{\frac{1}{2}}\left[\beta s+V^{2}(\beta s)\right] \\
& =-\varepsilon^{\frac{1}{3}} \beta^{3} V(\beta s)\left[1+\varepsilon^{\frac{2}{3}} \mathscr{O}\left(s^{2} V^{-2}\right)\right]^{\frac{1}{2}}\left[\beta s+V^{2}(\beta s)\right] \\
\operatorname{via}(68) & =-\varepsilon^{\frac{1}{3}} \beta^{3} V(\beta s)\left[1+\varepsilon^{\frac{2}{3}} \mathscr{O}(|s|)\right]\left[\beta s+V^{2}(\beta s)\right] \\
\operatorname{via}(68),(69) & =-\varepsilon^{\frac{1}{3}} \beta^{3} V(\beta s)\left[\beta s+V^{2}(\beta s)\right]+\mathscr{O}\left(\varepsilon|s|^{-\frac{1}{2}}\right),
\end{aligned}
$$


uniformly, as $\varepsilon \rightarrow 0$. Moreover, in the same region, we have:

$$
\begin{aligned}
\left(\tilde{u}_{\text {out }}\right)_{s}= & \frac{\varepsilon^{\frac{2}{3}}}{2}\left[a_{t}\left(\varepsilon^{\frac{2}{3}} s, \varepsilon^{\frac{2}{3}} z\right)+\beta^{3}+2 \beta^{3} V_{x}(\beta s) V(\beta s)\right] \\
& \times\left[a\left(\varepsilon^{\frac{2}{3}} y\right)+\varepsilon^{\frac{2}{3}} \beta^{3} s+\varepsilon^{\frac{2}{3}} \beta^{2} V^{2}(\beta s)\right]^{-\frac{1}{2}}, \\
\left(\tilde{u}_{\text {out }}\right)_{s s}= & \frac{\varepsilon^{\frac{2}{3}}}{2}\left[\varepsilon^{\frac{2}{3}} a_{t t}\left(\varepsilon^{\frac{2}{3}} s, \varepsilon^{\frac{2}{3}} z\right)+2 \beta^{4}\left(V_{x x} V+V_{x}^{2}\right)\right] \\
& \times\left[a\left(\varepsilon^{\frac{2}{3}} y\right)+\varepsilon^{\frac{2}{3}} \beta^{3} s+\varepsilon^{\frac{2}{3}} \beta^{2} V^{2}\right]^{-\frac{1}{2}} \\
& -\frac{\varepsilon^{\frac{4}{3}}}{4}\left[a_{t}\left(\varepsilon^{\frac{2}{3}} s, \varepsilon^{\frac{2}{3}} z\right)+\beta^{3}+2 \beta^{3} V_{x} V\right]^{2} \\
& \times\left[a\left(\varepsilon^{\frac{2}{3}} y\right)+\varepsilon^{\frac{2}{3}} \beta^{3} s+\varepsilon^{\frac{2}{3}} \beta^{2} V^{2}\right]^{-\frac{3}{2}}, \\
\left(\tilde{u}_{\text {out }}\right)_{z}= & \frac{1}{2}\left[\varepsilon^{\frac{2}{3}} a_{\theta}+3 \varepsilon^{\frac{4}{3}} \beta^{\prime} \beta^{2} s+2 \varepsilon^{\frac{4}{3}} \beta^{\prime} \beta V^{2}+2 \varepsilon^{\frac{4}{3}} \beta^{\prime} \beta^{2} s V_{x} V\right] \\
& \times\left[a+\varepsilon^{\frac{2}{3}} \beta^{3} s+\varepsilon^{\frac{2}{3}} \beta^{2} V^{2}\right]^{-\frac{1}{2}},
\end{aligned}
$$

and

$$
\begin{aligned}
\left(\tilde{u}_{\text {out }}\right)_{z z}= & \frac{1}{2}\left[\varepsilon^{\frac{4}{3}} a_{\theta \theta}+6 \varepsilon^{2}\left(\beta^{\prime}\right)^{2} \beta s+3 \varepsilon^{2} \beta^{\prime \prime} \beta^{2} s+2 \varepsilon^{2} V^{2}\left(\beta^{\prime \prime} \beta+\left(\beta^{\prime}\right)^{2}\right)\right. \\
& \left.+8 \varepsilon^{2}\left(\beta^{\prime}\right)^{2} \beta s V_{x} V+2 \varepsilon^{2} \beta^{\prime \prime} \beta^{2} s V_{x} V+2 \varepsilon^{2}\left(\beta^{\prime}\right)^{2} \beta^{2} s^{2}\left(V_{x}^{2}+V_{x x} V\right)\right] \\
& \times\left[a+\varepsilon^{\frac{2}{3}} \beta^{3} s+\varepsilon^{\frac{2}{3}} \beta^{2} V^{2}\right]^{-\frac{1}{2}} \\
& -\frac{1}{4}\left[\varepsilon^{\frac{2}{3}} a_{\theta}+3 \varepsilon^{\frac{4}{3}} \beta^{\prime} \beta^{2} s+2 \varepsilon^{\frac{4}{3}} \beta^{\prime} \beta\left(V^{2}+\beta s V_{x} V\right)\right]^{2} \\
& \times\left[a+\varepsilon^{\frac{2}{3}} \beta^{3} s+\varepsilon^{\frac{2}{3}} \beta^{2} V^{2}\right]^{-\frac{3}{2}}
\end{aligned}
$$

where the functions $a, a_{\theta} a_{\theta \theta}$ are evaluated at $\varepsilon^{\frac{2}{3}} y, V$ at $\beta s$, and $\beta$ at $\varepsilon^{\frac{2}{3}} z$. Estimating as in (92), we conclude that in the region described by $\left\{-\delta \varepsilon^{-\frac{2}{3}} \leqq \beta s \leqq-L, z \in\right.$ $\left.\left[0, \varepsilon^{-\frac{2}{3}} \ell_{\varepsilon}\right)\right\}$ we have:

$$
\begin{aligned}
\left(\tilde{u}_{\text {out }}\right)_{s} & =\varepsilon^{\frac{1}{3}} \beta^{2} V_{x}(\beta s)+\mathscr{O}\left(\varepsilon|s|^{\frac{1}{2}}\right),\left(\tilde{u}_{\text {out }}\right)_{s s}=\varepsilon^{\frac{1}{3}} \beta^{3} V_{x x}(\beta s)+\mathscr{O}\left(\varepsilon|s|^{-\frac{1}{2}}\right), \\
\left(\tilde{u}_{\text {out }}\right)_{z}=\mathscr{O}\left(\varepsilon|s|^{\frac{1}{2}}\right), & \left(\tilde{u}_{\text {out }}\right)_{z z}=\mathscr{O}\left(\varepsilon|s|^{-\frac{1}{2}}\right),
\end{aligned}
$$

uniformly as $\varepsilon \rightarrow 0$. We point out that, when estimating derivatives in $z$, we also made use of the bound

$$
\left|a_{\theta}\left(\varepsilon^{\frac{2}{3}} s, \varepsilon^{\frac{2}{3}} z\right)\right| \leqq C \varepsilon^{\frac{2}{3}}|s|
$$


which follows directly from (33). In order to elucidate the various cancelations of powers of $|s|$ involved, let us carefully present the proof of one of the estimates in (97):

$$
\begin{aligned}
\left|\left(\tilde{u}_{\text {out }}\right)_{z z}\right| \leqq & C\left(\varepsilon^{\frac{4}{3}}+\varepsilon^{2}|s|+\varepsilon^{2}|s||s|^{-\frac{1}{2}}|s|^{\frac{1}{2}}+\varepsilon^{2} s^{2}\left(|s|^{-1}+|s|^{-\frac{3}{2}}|s|^{\frac{1}{2}}\right)\right) \varepsilon^{-\frac{1}{3}}|s|^{-\frac{1}{2}} \\
& +C\left(\varepsilon^{\frac{4}{3}}|s|+\varepsilon^{\frac{4}{3}}|s||s|^{-\frac{1}{2}}|s|^{\frac{1}{2}}\right)^{2} \varepsilon^{-1}|s|^{-\frac{3}{2}} \\
\leqq & C\left(\varepsilon^{\frac{4}{3}}+\varepsilon^{2}|s|\right) \varepsilon^{-\frac{1}{3}}|s|^{-\frac{1}{2}}+C \varepsilon^{\frac{8}{3}}|s|^{2} \varepsilon^{-1}|s|^{-\frac{3}{2}} \\
\leqq & C \varepsilon|s|^{-\frac{1}{2}}+C \varepsilon^{\frac{5}{3}}|s|^{\frac{1}{2}} \leqq C \varepsilon|s|^{-\frac{1}{2}}+C \varepsilon^{\frac{5}{3}}|s||s|^{-\frac{1}{2}} \leqq C \varepsilon|s|^{-\frac{1}{2}}
\end{aligned}
$$

In $\left\{-2 \delta \varepsilon^{-\frac{2}{3}} \leqq \beta s \leqq-\delta \varepsilon^{-\frac{2}{3}}, z \in\left[0, \varepsilon^{-\frac{2}{3}} \ell_{\varepsilon}\right)\right\}$, making again use of (68), (69), we can show that

$$
\begin{aligned}
& \tilde{u}_{\text {out }}^{2}-a\left(\varepsilon^{\frac{2}{3}} y\right)=\mathscr{O}\left(\varepsilon^{2}\right),\left(\tilde{u}_{\text {out }}\right)_{s}=\mathscr{O}\left(\varepsilon^{\frac{2}{3}}\right),\left(\tilde{u}_{\text {out }}\right)_{s s}=\mathscr{O}\left(\varepsilon^{\frac{4}{3}}\right), \\
&\left(\tilde{u}_{\text {out }}\right)_{z}=\mathscr{O}\left(\varepsilon^{\frac{2}{3}}\right),\left(\tilde{u}_{\text {out }}\right)_{z z}=\mathscr{O}\left(\varepsilon^{\frac{4}{3}}\right),
\end{aligned}
$$

uniformly as $\varepsilon \rightarrow 0$. Estimate (88) now follows from (56), (59), the fact that $V$ solves (65), (69), (92), and (97); estimate (89) follows at once from (56), (59), (87), and (98). The first relation in (90) can be shown by working as in (92). The remaining estimates in (90) follow directly from (68), (69), (80), (97), and the fact that

$$
u_{y_{1}}^{2}+u_{y_{2}}^{2}=u_{s}^{2}+\left(1+\varepsilon^{\frac{2}{3}} k s\right)^{2} u_{z}^{2}
$$

for $y=\left(y_{1}, y_{2}\right) \in\left\{(s, z)|| s \mid \leqq \delta_{0} \varepsilon^{-\frac{2}{3}}, z \in\left[0, \varepsilon^{-\frac{2}{3}} \ell_{\varepsilon}\right)\right\}$, and any smooth function $u$ defined in this region [recall (55)]. Finally, estimate (91) follows readily from (33), and (69).

The proof of the proposition is complete.

\subsection{The Approximate Solution $u_{\text {ap }}$}

In this subsection we will construct a smooth approximate solution for problem (51) that is valid in all of $\mathbb{R}^{2}$. This will be achieved by smoothly interpolating between $\tilde{u}_{\text {out }}$ and $u_{\text {in }}$ in $\tilde{\mathscr{D}}_{\varepsilon}$, and between $u_{\text {in }}$ and zero in $\mathbb{R}^{2} \backslash \tilde{\mathscr{D}}_{\varepsilon}$.

To this end, we need to introduce one more smooth cutoff function:

$$
\rho_{L}(x)= \begin{cases}0, & x \geqq-L, \\ 1, & x \leqq-2 L .\end{cases}
$$

We can now define our approximate solution for (51) as

$$
u_{\text {ap }}= \begin{cases}\tilde{u}_{\text {out }} & \text { in } \tilde{\mathscr{D}}_{\varepsilon} \backslash\{-2 L<x<0\}, \\ u_{\text {in }}+\rho_{L}(x)\left(\tilde{u}_{\text {out }}-u_{\text {in }}\right) & \text { in }\left\{-2 L \leqq x \leqq \delta \varepsilon^{-\frac{2}{3}}\right\} \\ \chi_{10 \delta}(x) u_{\text {in }} & \text { everywhere else }\end{cases}
$$

where $u_{\text {in }}, \chi_{\delta}, \tilde{u}_{\text {out }}$ were defined in (80), (86), and (87) respectively.

The following proposition contains the main estimates concerning $u_{\text {ap }}$. 
Proposition 4. The approximate solution $u_{\text {ap }}$ satisfies

$$
\Delta u_{\text {ap }}-\varepsilon^{-\frac{2}{3}} u_{\text {ap }}\left(u_{\text {ap }}^{2}-a\left(\varepsilon^{\frac{2}{3}} y\right)\right)=\mathscr{O}\left(\varepsilon(|s|+1)^{-\frac{1}{2}}\right)
$$

uniformly in $\left\{-\delta \varepsilon^{-\frac{2}{3}} \leqq \beta s \leqq 0, z \in\left[0, \varepsilon^{-\frac{2}{3}} \ell_{\varepsilon}\right)\right\}$,

$$
\Delta u_{\text {ap }}-\varepsilon^{-\frac{2}{3}} u_{\text {ap }}\left(u_{\text {ap }}^{2}-a\left(\varepsilon^{\frac{2}{3}} y\right)\right)=\mathscr{O}\left(\varepsilon^{\frac{4}{3}}\right)
$$

uniformly in $\tilde{\mathscr{D}}_{\varepsilon} \backslash\left\{-\delta \varepsilon^{-\frac{2}{3}} \leqq \beta s<0, z \in\left[0, \varepsilon^{-\frac{2}{3}} \ell_{\varepsilon}\right)\right\}$,

$$
\Delta u_{\text {ap }}-\varepsilon^{-\frac{2}{3}} u_{\text {ap }}\left(u_{\text {ap }}^{2}-a\left(\varepsilon^{\frac{2}{3}} y\right)\right)=\mathscr{O}\left(\varepsilon \mathrm{e}^{-c|s|^{\frac{3}{2}}}\right)
$$

uniformly in $\left\{0 \leqq \beta s \leqq 2 \delta \varepsilon^{-\frac{2}{3}}, z \in\left[0, \varepsilon^{-\frac{2}{3}} \ell_{\varepsilon}\right)\right\}$, as $\varepsilon \rightarrow 0$, and

$$
u_{\mathrm{ap}}=0 \text { everywhere else. }
$$

If $\varepsilon>0$ is sufficiently small, we have

$$
3 u_{\text {ap }}^{2}-a\left(\varepsilon^{\frac{2}{3}} y\right) \geqq \begin{cases}c \varepsilon^{\frac{2}{3}}(1+|x|), & \text { if }|x| \leqq \delta \varepsilon^{-\frac{2}{3}}, \\ c+c\left|\varepsilon^{\frac{2}{3}} y\right|^{p}, & \text { otherwise. }\end{cases}
$$

Proof. We will first consider relations (102)-(105). In view of estimates (82), (83), (88), (89), and recalling the super-exponential decay of $V$ as $x \rightarrow \infty$, it just remains to show the validity of (102) in the interpolating region described by $\left\{-2 L \leqq \beta s \leqq-L, z \in\left[0, \varepsilon^{-\frac{2}{3}} \ell_{\varepsilon}\right)\right\}$. There, we have

$$
\begin{aligned}
& \Delta u_{\text {ap }}-\varepsilon^{-\frac{2}{3}} u_{\text {ap }}\left(u_{\text {ap }}^{2}-a\left(\varepsilon^{\frac{2}{3}} y\right)\right) \\
& =\Delta u_{\text {in }}-\varepsilon^{-\frac{2}{3}} u_{\text {in }}\left(u_{\text {in }}^{2}-a\left(\varepsilon^{\frac{2}{3}} y\right)\right)+\left(\Delta \rho_{L}\right)\left(\tilde{u}_{\text {out }}-u_{\text {in }}\right) \\
& \quad+2 \nabla \rho_{L} \nabla\left(\tilde{u}_{\text {out }}-u_{\text {in }}\right)+\rho_{L} \Delta\left(\tilde{u}_{\text {out }}-u_{\text {in }}\right) \\
& \quad-\varepsilon^{-\frac{2}{3}} u_{\text {in }}\left[3 \rho_{L}^{2}\left(\tilde{u}_{\text {out }}-u_{\text {in }}\right)^{2}+2 u_{\text {in }} \rho_{L}\left(\tilde{u}_{\text {out }}-u_{\text {in }}\right)\right] \\
& \quad-\varepsilon^{-\frac{2}{3}} \rho_{L}\left(\tilde{u}_{\text {out }}-u_{\text {in }}\right)\left[u_{\text {in }}^{2}-a\left(\varepsilon^{\frac{2}{3}} y\right)+\rho_{L}^{2}\left(\tilde{u}_{\text {out }}-u_{\text {in }}\right)^{2}\right],
\end{aligned}
$$

and the desired estimate follows via (82), (90), noting that $u_{\text {in }}^{2}, a\left(\varepsilon^{\frac{2}{3}} y\right)$ are of order $\varepsilon^{\frac{2}{3}}$ in this region.

The proof of lower bound (106) proceeds as follows: In the neighborhood described by $\{|x| \leqq 2 L\}$ of the curve $\tilde{\Gamma}_{\varepsilon}$ (with the obvious notation), by virtue of (33), (36), (90), (101), we find that

$$
\begin{aligned}
3 u_{\mathrm{ap}}^{2}-a\left(\varepsilon^{\frac{2}{3}} y\right) & =3 \varepsilon^{\frac{2}{3}} \beta^{2} V^{2}(x)+\mathscr{O}\left(\varepsilon^{\frac{4}{3}}\right)+\varepsilon^{\frac{2}{3}} \beta^{2} x+\mathscr{O}\left(\varepsilon^{\frac{4}{3}}\right) x^{2} \\
& =\varepsilon^{\frac{2}{3}} \beta^{2}\left(3 V^{2}(x)+x\right)+\mathscr{O}\left(\varepsilon^{\frac{4}{3}}\right)
\end{aligned}
$$

uniformly as $\varepsilon \rightarrow 0$. In $\tilde{\mathscr{D}}_{\varepsilon} \backslash\{-2 L<x<0\}$, we have $u_{\text {ap }}=\tilde{u}_{\text {out }}$ and, by (69), (87), we infer that

$$
\begin{aligned}
3 u_{\mathrm{ap}}^{2}-a\left(\varepsilon^{\frac{2}{3}} y\right) & =2 a\left(\varepsilon^{\frac{2}{3}} y\right)+3 \varepsilon^{\frac{2}{3}} \beta^{2} \chi_{\delta}(\beta s)\left(V^{2}(\beta s)+\beta s\right) \\
& =2 a\left(\varepsilon^{\frac{2}{3}} y\right)+\varepsilon^{\frac{2}{3}} \chi_{\delta}(\beta s) \mathscr{O}\left(|s|^{-2}\right)
\end{aligned}
$$


uniformly as $\varepsilon \rightarrow 0$. In points outside of the domain $\tilde{\mathscr{D}}_{\varepsilon} \cup\{0 \leqq x<2 L\}$, we plainly note that

$$
3 u_{\text {ap }}^{2}-a\left(\varepsilon^{\frac{2}{3}} y\right) \geqq-a\left(\varepsilon^{\frac{2}{3}} y\right) .
$$

The desired lower bound (106) now follows readily from the above three relations, via (4), (33), and (71), increasing $L>0$ if necessary.

The proof of the proposition is complete.

Remark 9. From the geometric singular perturbation viewpoint, recall Remark 6, matching is accomplished by employing a useful lemma on the flow past a "corner equilibrium" (see [189]). Manifolds of corner equilibria arise in blown-up geometric singular perturbation problems precisely where the inner and outer solutions must be matched. When such equilibria are normally hyperbolic, as in the onedimensional case of the problem at hand (see [190]), this lemma plays the same role in tracking the flow past them that the Exchange Lemma [131] plays at certain other manifolds of equilibria.

Remark 10. Our construction of $u_{\text {ap }}$ should also be applicable to the homogenized Gross-Pitaevskii equations considered in [162, Sec. 7].

\subsection{Mapping Properties of the Linearized Operator}

In this subsection we will invert the linearized operator

$$
\mathscr{L}(\varphi)=\Delta \varphi-\varepsilon^{-\frac{2}{3}}\left(3 u_{\text {ap }}^{2}-a\left(\varepsilon^{\frac{2}{3}} y\right)\right) \varphi
$$

in carefully chosen weighted spaces. The use of weighted spaces is a powerful technique in elliptic singular perturbation problems, and in many problems arising from geometry, see [171]. To the best of our knowledge, they are used here for the first time in singular perturbation problems involving corner layers. Actually, the weighted spaces that we will use are a variant of those considered in [179], and are motivated from relations (88), (106), keeping in mind that we ultimately wish to find a true solution of (51) near $u_{\text {ap }}$ via a perturbation argument.

Consider a smooth non-increasing function $g$ such that

$$
g(s)= \begin{cases}1, & s \geqq 0, \\ \left(\frac{\max \beta}{L}\right)^{\frac{3}{2}}\left(\frac{L}{\max \beta}-s\right)^{\frac{3}{2}}, & -\frac{2 \delta}{\min \beta} \varepsilon^{-\frac{2}{3}} \leqq s \leqq-\frac{L}{\max \beta}, \\ \left(\frac{\max \beta}{\min \beta}\right)^{\frac{3}{2}} L^{-\frac{3}{2}} \varepsilon^{-1}, & s \leqq-\frac{3 \delta}{\min \beta} \varepsilon^{-\frac{2}{3}},\end{cases}
$$

and

$$
\begin{aligned}
& 0 \leqq-g^{\prime} \leqq C L^{-1}, \quad\left|g^{\prime \prime}\right| \leqq C L^{-2}, \quad s \in\left[-\frac{L}{\max \beta}, 0\right] \\
& 0 \leqq-g^{\prime} \leqq C L^{-\frac{3}{2}} \varepsilon^{-\frac{1}{3}}, \quad\left|g^{\prime \prime}\right| \leqq C L^{-\frac{3}{2}} \varepsilon^{\frac{1}{3}}, \quad s \in\left[-\frac{3 \delta}{\min \beta} \varepsilon^{-\frac{2}{3}},-\frac{2 \delta}{\min \beta} \varepsilon^{-\frac{2}{3}}\right]
\end{aligned}
$$


where the constant $C$ is independent of small $\varepsilon$ and large $L$. Recalling (106), it is easy to check that we can fix an $L_{0}>0$ such that

$$
\begin{aligned}
\left|\frac{g^{\prime \prime}}{g}\right| & +2\left|\frac{g^{\prime}}{g}\right|^{2} \\
& \leqq C \varepsilon^{\frac{4}{3}}+C L^{-2} \leqq \frac{\varepsilon^{-\frac{2}{3}}}{2} \min _{\mathbb{R}^{2}}\left(3 u_{\text {ap }}^{2}-a\left(\varepsilon^{\frac{2}{3}} y\right)\right), \quad s \in \mathbb{R}, \text { if } L \geqq L_{0},
\end{aligned}
$$

provided $\varepsilon$ is sufficiently small ( $C$ in the above relation is independent of $\varepsilon, L$ ). Relations (107)-(109) imply that, for small $\varepsilon$, we have

$$
g^{\frac{2}{3}}(y) \leqq C \varepsilon^{-\frac{2}{3}}\left(3 u_{\text {ap }}^{2}-a\left(\varepsilon^{\frac{2}{3}} y\right)\right) \text { in } \mathbb{R}^{2},
$$

[here $g$ is viewed as a smooth function of $y$, which close to $\tilde{\Gamma}_{\varepsilon}$, in coordinates $(s, z)$, is given by (111), and otherwise equals the constants in (111)].

For $\varphi \in L^{\infty}\left(\mathbb{R}^{2}\right)$, we define the following weighted norms:

$$
\|\varphi\|_{\frac{3}{2}} \equiv\|g \varphi\|_{L^{\infty}\left(\mathbb{R}^{2}\right)} \quad \text { and } \quad\|\varphi\|_{\frac{1}{2}} \equiv\left\|g^{\frac{1}{3}} \varphi\right\|_{L^{\infty}\left(\mathbb{R}^{2}\right)}
$$

(We utilized this notation because $g$ behaves qualitatively like $(-s)^{\frac{3}{2}}$ for $s<0$.)

We also consider the Banach space

$$
\mathscr{X} \equiv\left\{\varphi:\|\varphi\| \mathscr{X} \equiv\left\|\mathrm{e}^{\left|\varepsilon^{\frac{2}{3}} y\right|} \varphi\right\|_{L^{\infty}\left(\mathbb{R}^{2}\right)}\right\}<\infty .
$$

The following proposition will be used essentially in the sequel.

Proposition 5. If $\varepsilon$ is sufficiently small, given $f \in \mathscr{X} \cap C^{\alpha}\left(\mathbb{R}^{2}\right), 0<\alpha<1$, there exists a unique $\varphi \in \mathscr{X} \cap C^{2+\alpha}\left(\mathbb{R}^{2}\right)$ such that

$$
\mathscr{L}(\varphi)=f,
$$

where the linear operator $\mathscr{L}$ was defined in (110). Furthermore, we have

$$
\|\varphi\| \mathscr{X} \leqq C\|f\| \mathscr{X},
$$

and

$$
\|\varphi\|_{\frac{3}{2}} \leqq C\|f\|_{\frac{1}{2}}
$$

for some constant $C$ independent of $f, \varepsilon$.

Proof. The first assertion of the proposition, including estimate (119), follows in a standard way: It follows from (106), the maximum principle, and elliptic regularity theory [109], that there exists a solution $\varphi \in C^{2+\alpha}\left(\mathbb{R}^{2}\right)$ of (118) such that

$$
\|\varphi\|_{L^{\infty}\left(\mathbb{R}^{2}\right)} \leqq C\|f\|_{L^{\infty}\left(\mathbb{R}^{2}\right)},
$$

for some constant $C$ independent of $f, \varepsilon$. This is easy to prove, though it is difficult to find a good reference. (For example, one can first solve equation (118) in a ball $B_{R}$ 
with Dirichlet boundary conditions to obtain a solution $\varphi_{R}$ such that $\left\|\varphi_{R}\right\|_{L^{\infty}\left(B_{R}\right)} \leqq$ $C\|f\|_{L^{\infty}\left(B_{R}\right)}$, for some $C$ independent of $f, \varepsilon, R$, extend $\varphi_{R}$ in $L^{\infty}\left(\mathbb{R}^{2}\right)$ to be zero outside of $B_{R}$, and prove that $\varphi_{R_{i}}$, for some $R_{i} \rightarrow \infty$, converge uniformly in compact sets of $\mathbb{R}^{2}$ to a solution $\varphi$ of (118) that satisfies (121).) Then, a standard barrier argument, using as barrier the function

$$
\bar{\varphi}_{\tau}(y)=\tau \mathrm{e}^{\left|\varepsilon^{2 / 3} y\right|}+\|\varphi\|_{L^{\infty}\left(\mathbb{R}^{2}\right)} \mathrm{e}^{\left(R-\left|\varepsilon^{2 / 3} y\right|\right)}, \quad|y| \geqq \frac{R}{\varepsilon^{2 / 3}},
$$

where $R>0$ is chosen large, $\tau>0$ arbitrary, yields that $|\varphi(y)| \leqq \bar{\varphi}_{\tau}(y),|y| \geqq$ $R \varepsilon^{-2 / 3}$, provided $\varepsilon$ is small enough so that (106) holds. Lastly, letting $\tau \rightarrow 0$, and recalling (121), we conclude that estimate (119) holds true (see also [80, Lemma 7.3]).

Let

$$
\psi=g \varphi,
$$

then, thanks to (56), (59), it is easy to see that, with the obvious notation, we have

$$
\begin{aligned}
& \Delta_{y} \psi-2 \frac{g^{\prime}}{g} \psi_{s}-\varepsilon^{-\frac{2}{3}}\left(3 u_{\text {ap }}^{2}-a\left(\varepsilon^{\frac{2}{3}} y\right)\right) \psi-\frac{g^{\prime \prime}}{g} \psi \\
& +2\left(\frac{g^{\prime}}{g}\right)^{2} \psi-\varepsilon^{\frac{2}{3}} a_{0} \frac{g^{\prime}}{g} \psi=g f,
\end{aligned}
$$

$y \in \mathbb{R}^{2}$. Since $\psi \rightarrow 0$ as $|y| \rightarrow \infty$, recalling (106), (114), (115), we can apply the maximum principle to show that, for small $\varepsilon$,

$$
|\psi(y)| \leqq C\left\|g^{\frac{1}{3}} f\right\|_{L^{\infty}\left(\mathbb{R}^{2}\right)}, \quad y \in \mathbb{R}^{2},
$$

for some constant $C$ independent of $\varepsilon, f$. We also used the fact that $\psi_{s}=0$ whenever $\nabla_{y} \psi=0$, which follows immediately from relation (99). The desired bound (120) now follows at once from (116), (123), and the above relation.

The proof of the proposition is complete.

Remark 11. In the above proof, we made essential use of lower bound (106) whose proof, we recall, relied crucially on lower bound (71) which was established recently in [104]. However, as we have remarked in the proof of Proposition 1, its proof is rather involved and technical. In Appendix A, we will provide a more natural and flexible proof of Proposition 5 without assuming knowledge of (71). Instead, we will make use of the asymptotic stability of the Hastings-McLeod solution $V$, namely the fact that the principal eigenvalue of the operator $-\mathscr{M}$, defined in (70), is strictly positive. This follows immediately by testing the corresponding eigenvalue problem by $V_{x}<0$, see [191] and Proposition 10 herein. In other words, we will rely on the lower bound:

$$
-\int_{-\infty}^{\infty} \phi \mathscr{M}(\phi) \mathrm{d} x \geqq c \int_{-\infty}^{\infty} \phi^{2} \mathrm{~d} x \quad \forall \phi \in C_{0}^{\infty}(\mathbb{R}),
$$

for some constant $c>0$, which clearly is much "softer" than (71). We point out that the validity of (71) was not needed (nor known) in references [190,191] which dealt 
with related one-dimensional problems. Actually, in one-dimensional or radially symmetric cases, for Proposition 5 to hold, it suffices to know that zero is not in the kernel of $\mathscr{M}$. However, this may not be true in general higher dimensional problems due to a possible resonance phenomenon (see $[78,135])$.

\subsection{Existence of a Solution}

Here we will use the contraction mapping principle in order to capture a genuine solution $u_{\varepsilon}$ of (51) close to the approximate solution $u_{\text {ap }}$, provided $\varepsilon$ is sufficiently small.

Proposition 6. If $\varepsilon$ is sufficiently small, then there exists a solution $u_{\varepsilon}$ of (51) such that

$$
\begin{gathered}
\left\|u_{\varepsilon}-u_{\text {ap }}\right\|_{\mathscr{X}} \leqq C \varepsilon \\
\left\|u_{\varepsilon}-u_{\text {ap }}\right\|_{\frac{3}{2}} \leqq C \varepsilon
\end{gathered}
$$

and

$$
\left|u_{\varepsilon}(y)-u_{\text {ap }}(y)\right| \leqq C \varepsilon \mathrm{e}^{-c s}, \quad y \in\left\{0 \leqq \beta s \leqq 2 \delta \varepsilon^{-\frac{2}{3}}, z \in\left[0, \varepsilon^{-\frac{2}{3}} \ell_{\varepsilon}\right)\right\},
$$

where the norms involved were defined in (116) and (117).

Proof. We seek a true solution of problem (51) in the form

$$
u=u_{\mathrm{ap}}+\varphi .
$$

In order for $u$ to satisfy the equation in (51), we readily find that the correction $\varphi$ has to solve

$$
\mathscr{L}(\varphi)=E+N(\varphi)
$$

where the linear operator $\mathscr{L}$ was defined in (110),

$$
E=-\Delta u_{\text {ap }}+\varepsilon^{-\frac{2}{3}} u_{\text {ap }}\left(u_{\text {ap }}^{2}-a\left(\varepsilon^{\frac{2}{3}} y\right)\right),
$$

and

$$
N(\varphi)=3 \varepsilon^{-\frac{2}{3}} u_{\mathrm{ap}} \varphi^{2}+\varepsilon^{-\frac{2}{3}} \varphi^{3} .
$$

Given $M>0$ to be determined (independently of $\varepsilon$ ), we consider the closed bounded subset of $\mathscr{X}$ defined by

$$
\mathscr{Y}=\left\{\varphi \in \mathscr{X}:\|\varphi\|_{\mathscr{X}} \leqq M \varepsilon,\|\varphi\|_{\frac{3}{2}} \leqq M \varepsilon\right\}
$$

We will prove that, if $M$ is chosen sufficiently large, the operator $\mathbb{P}$, defined from $\mathscr{X} \cap C^{2+\alpha}\left(\mathbb{R}^{2}\right)$ into $\mathscr{X} \cap C^{2+\alpha}\left(\mathbb{R}^{2}\right)$ by

$$
\mathbb{P}(\varphi)=\mathscr{L}^{-1}(E+N(\varphi)),
$$


maps $\mathscr{Y}$ into itself, and is a contraction with respect to the $\mathscr{X}$-norm, provided $\varepsilon$ is sufficiently small. Note that $\mathscr{L}^{-1}$ is well defined by virtue of Proposition 5 . In view of the estimates of Proposition 4, (111), (116), and (117), for small $\varepsilon>0$, we have

$$
\|E\| \mathscr{X} \leqq C \varepsilon \text { and }\|E\|_{\frac{1}{2}} \leqq C \varepsilon
$$

Furthermore, there exists a constant $C>0$ such that, for all $\varphi_{1}, \varphi_{2}, \varphi \in \mathscr{X}$, the following relations hold pointwise:

$$
\begin{aligned}
& \left|N\left(\varphi_{1}\right)-N\left(\varphi_{2}\right)\right| \leqq C \varepsilon^{-\frac{2}{3}}\left(\varphi_{1}^{2}+\varphi_{2}^{2}\right)\left|\varphi_{1}-\varphi_{2}\right| \\
& \quad+C \varepsilon^{-\frac{2}{3}}\left|u_{\mathrm{ap}}\right|\left(\left|\varphi_{1}\right|+\left|\varphi_{2}\right|\right)\left|\varphi_{1}-\varphi_{2}\right| \\
& \left|g^{\frac{1}{3}} N(\varphi)\right| \leqq C \varepsilon^{-\frac{2}{3}}|g \varphi|^{3}+C \varepsilon^{-\frac{2}{3}}\left|u_{\mathrm{ap}}\right||g \varphi|^{2}
\end{aligned}
$$

for every $y \in \mathbb{R}^{2}$ (recall that $g \geqq 1$ ). If $\varphi \in \mathscr{Y}$, by Proposition 5, (133), and (134), we obtain that

$$
\begin{aligned}
\|\mathbb{P}(\varphi)\|_{\mathscr{X}} & \leqq C\|E\| \mathscr{X}+C\|N(\varphi)\| \mathscr{X} \\
& \leqq C \varepsilon+C \varepsilon^{-\frac{2}{3}}\|\varphi\|_{\mathscr{X}}^{3}+C \varepsilon^{-\frac{2}{3}}\|\varphi\|_{\mathscr{X}}^{2} \\
& \leqq C \varepsilon+C M^{3} \varepsilon^{\frac{7}{3}}+C M^{2} \varepsilon^{\frac{4}{3}}
\end{aligned}
$$

and

$$
\begin{aligned}
\|\mathbb{P}(\varphi)\|_{\frac{3}{2}} & \leqq C\|E\|_{\frac{1}{2}}+C\|N(\varphi)\|_{\frac{1}{2}} \\
& \leqq C \varepsilon+C \varepsilon^{-\frac{2}{3}}\|\varphi\|_{\frac{3}{2}}^{3}+C \varepsilon^{-\frac{2}{3}}\|\varphi\|_{\frac{3}{2}}^{2} \\
& \leqq C \varepsilon+C M^{3} \varepsilon^{\frac{7}{3}}+C M^{2} \varepsilon^{\frac{4}{3}},
\end{aligned}
$$

where $C$ is independent of $\varphi, M$ and small $\varepsilon$. We conclude that, if $M$ is chosen sufficiently large, the operator $\mathbb{P}$ maps $\mathscr{Y}$ into itself, provided $\varepsilon$ is sufficiently small. We have to prove that $\mathbb{P}$ is a contraction from $\mathscr{Y}$ into itself with respect to the $\mathscr{X}$-norm. Let $\varphi_{1}, \varphi_{2} \in \mathscr{Y}$. As before, we have

$$
\begin{aligned}
\left\|\mathbb{P}\left(\varphi_{1}\right)-\mathbb{P}\left(\varphi_{2}\right)\right\| \mathscr{X} & \leqq C\left\|N\left(\varphi_{1}\right)-N\left(\varphi_{2}\right)\right\|_{\mathscr{X}} \\
& \leqq C \varepsilon^{\frac{1}{3}}\left\|\varphi_{1}-\varphi_{2}\right\| \mathscr{X} .
\end{aligned}
$$

Hence, for small $\varepsilon>0$, the operator $\mathbb{P}: \mathscr{Y} \rightarrow \mathscr{Y}$ becomes a contraction with respect to the $\mathscr{X}$-norm. So, recalling that $\mathscr{Y}$ is closed in $\mathscr{X}$, it has a unique fixed point $\varphi_{*} \in \mathscr{Y}$, thanks to the contraction mapping theorem (see for instance [109]). It is clear that the function $u_{\varepsilon} \equiv u_{\text {ap }}+\varphi_{*}$ satisfies the elliptic equation in (51), and estimates (126)-(127).

Next, we show that $u_{\varepsilon}$ is positive, and consequently solves problem (51). In the neighborhood described by $\{|x| \leqq 2 L\}$ of the curve $\tilde{\Gamma}_{\varepsilon}$, recalling (80), (90), (101), and that $\varphi_{*} \in \mathscr{Y}$, we have

$$
u_{\varepsilon}=u_{\text {in }}+\mathscr{O}(\varepsilon)=\varepsilon^{\frac{1}{3}} \beta V(x)+\mathscr{O}(\varepsilon) \geqq c \varepsilon^{\frac{1}{3}}
$$


uniformly as $\varepsilon \rightarrow 0$. In the domain $\tilde{\mathscr{D}}_{\varepsilon} \backslash\{-2 L<x<0\}$, thanks to (69), (87), we have

$$
u_{\varepsilon}=\tilde{u}_{\text {out }}+\mathscr{O}(\varepsilon)=\left[a\left(\varepsilon^{\frac{2}{3}} y\right)+\mathscr{O}\left(\varepsilon^{\frac{2}{3}} L^{-2}\right)\right]^{\frac{1}{2}}+\mathscr{O}(\varepsilon) \stackrel{(33)}{\geqq} c \varepsilon^{\frac{1}{3}}
$$

uniformly as $\varepsilon \rightarrow 0$ (having increased $L$ if necessary). It remains to consider points outside of the domain $\tilde{\mathscr{D}}_{\varepsilon} \cup\{0 \leqq x<2 L\}$, where $u$ solves an equation of the form

$$
\Delta u-p(y) u=0, \quad \text { where } p \geqq c,
$$

[recall (4), (33)]. The positivity of $u$ in this region follows directly from the maximum principle, and the fact that we have already shown that $u \geqq c \varepsilon^{\frac{1}{3}}$ on the boundary described by the closed curve $\{x=2 L\}$.

It remains to establish the validity of (126)-(128). Since $u_{\varepsilon}-u_{\text {ap }}=\varphi_{*} \in \mathscr{Y}$, we see that (126), (127) hold. Finally, we will show (128) by suitably modifying the proof of Lemma 2 in [38] (see also [95, pg. 230] and [125, Lem. 2.2]). From (104), (105), (106), (126), and (129), we find that $\varphi_{*}$ satisfies

$$
\Delta \varphi_{*}-P(y) \varphi_{*}=\mathscr{O}\left(\varepsilon \mathrm{e}^{-\frac{\sqrt{c}}{2} s}\right), \quad \text { where } P(y) \geqq c,
$$

uniformly in $\left\{0 \leqq \beta s \leqq 4 \delta \varepsilon^{-\frac{2}{3}}, z \in\left[0, \varepsilon^{-\frac{2}{3}} \ell_{\varepsilon}\right)\right\}$, as $\varepsilon \rightarrow 0$. The reason for choosing, in the righthand side, a decay rate strictly less than $\sqrt{c}$ is to facilitate our next argument. Let

$$
\bar{\varphi}(y)=\bar{M} \varepsilon\left\{\mathrm{e}^{-\frac{\sqrt{c}}{2} s}+\mathrm{e}^{\frac{\sqrt{c}}{2}\left(s-4 \delta \varepsilon^{-\frac{2}{3}} \beta^{-1}\right)}\right\},
$$

$y \in\left\{0 \leqq \beta s \leqq 4 \delta \varepsilon^{-\frac{2}{3}}, z \in\left[0, \varepsilon^{-\frac{2}{3}} \ell_{\varepsilon}\right)\right\}$, where the value of the large constant $\bar{M}>0$ will soon be fixed independently of small $\varepsilon$. By virtue of (126), (136), we can choose a large $\bar{M}>0$ such that

$$
\left\{\begin{array}{l}
-\Delta\left(\bar{\varphi}-\varphi_{*}\right)+P(y)\left(\bar{\varphi}-\varphi_{*}\right)>0, \quad y \in\left\{0 \leqq \beta s \leqq 4 \delta \varepsilon^{-\frac{2}{3}}, z \in\left[0, \varepsilon^{-\frac{2}{3}} \ell_{\varepsilon}\right)\right\} \\
\bar{\varphi}-\varphi_{*}>0 \text { on }\{s=0\} \cup\left\{\beta s=4 \delta \varepsilon^{-\frac{2}{3}}\right\}
\end{array}\right.
$$

if $\varepsilon$ is sufficiently small. Now, by the second estimate in (136), and the maximum principle, we deduce that

$$
\bar{\varphi}-\varphi_{*}>0 \quad \text { if } 0 \leqq \beta s \leqq 4 \delta \varepsilon^{-\frac{2}{3}}
$$

In turn, the above estimate readily implies the validity of (128).

The proof of the proposition is complete.

The following estimates hold: 
Corollary 1. The solution $u_{\varepsilon}$ of (51), constructed in Proposition 6, satisfies

$$
u_{\varepsilon}=\varepsilon^{\frac{1}{3}} \beta\left(\varepsilon^{\frac{2}{3}} z\right) V\left(\beta\left(\varepsilon^{\frac{2}{3}} z\right) s\right)+\mathscr{O}\left(\varepsilon|s|^{\frac{3}{2}}+\varepsilon\right)
$$

uniformly in $\left\{-\delta \varepsilon^{-\frac{2}{3}} \leqq \beta s \leqq 0, z \in\left[0, \varepsilon^{-\frac{2}{3}} \ell_{\varepsilon}\right)\right\}$,

$$
u_{\varepsilon}=\varepsilon^{\frac{1}{3}} \beta\left(\varepsilon^{\frac{2}{3}} z\right) V\left(\beta\left(\varepsilon^{\frac{2}{3}} z\right) s\right)+\mathscr{O}\left(\varepsilon \mathrm{e}^{-c s}\right)
$$

uniformly in $\left\{0 \leqq \beta s \leqq 2 \delta \varepsilon^{-\frac{2}{3}}, z \in\left[0, \varepsilon^{-\frac{2}{3}} \ell_{\varepsilon}\right)\right\}$,

$$
u_{\varepsilon}=\sqrt{a\left(\varepsilon^{\frac{2}{3}} y\right)}+\mathscr{O}\left(\varepsilon^{\frac{1}{3}}|s|^{-\frac{5}{2}}\right)
$$

uniformly in $\left\{-\delta \varepsilon^{-\frac{2}{3}} \leqq \beta s \leqq-2 L, \quad z \in\left[0, \varepsilon^{-\frac{2}{3}} \ell_{\varepsilon}\right)\right\}$,

$$
u_{\varepsilon}=\sqrt{a\left(\varepsilon^{\frac{2}{3}} y\right)}+\mathscr{O}\left(\varepsilon^{2}\right)
$$

uniformly in $\tilde{\mathscr{D}}_{\varepsilon} \backslash\left\{-\delta \varepsilon^{-\frac{2}{3}}<\beta s<0, z \in\left[0, \varepsilon^{-\frac{2}{3}} \ell_{\varepsilon}\right)\right\}$, and

$$
0<u_{\varepsilon} \leqq C \varepsilon^{\frac{1}{3}} \exp \left\{-c \operatorname{dist}\left(y, \tilde{\mathscr{D}}_{\varepsilon}\right)\right\}
$$

in $\mathbb{R}^{2} \backslash \tilde{\mathscr{D}}_{\varepsilon}$, as $\varepsilon \rightarrow 0$.

Proof. Estimates (137), (138) follow readily from (80), the first relation in (90), (101), (126), and (128). From (91), (101), (111), (127), for small $\varepsilon>0$, we have

$$
\left|u_{\varepsilon}-\sqrt{a\left(\varepsilon^{\frac{2}{3}} y\right)}\right| \leqq C \varepsilon|s|^{-\frac{3}{2}}+C \varepsilon^{\frac{1}{3}}|s|^{-\frac{5}{2}} \leqq C \varepsilon^{\frac{1}{3}}|s|^{-\frac{5}{2}}
$$

in $\left\{-\delta \varepsilon^{-\frac{2}{3}} \leqq \beta s \leqq-2 L, z \in\left[0, \varepsilon^{-\frac{2}{3}} \ell_{\varepsilon}\right)\right\}$, and estimate (139) follows immediately. Estimate (140) follows at once from (87), (101), (111), and (127). Finally, estimate (141) follows readily from (135), (138), arguing as we did for the proof of (128) (but here we need to cover $\mathbb{R}^{2}$ by a finite number of disjoint annular domains, surrounding $\mathscr{D}_{0}$, and the exterior of a large ball), see also [95, pg. 230].

The proof of the corollary is complete.

Remark 12. Notice that the nonlinear terms in (129) are of cubic-like order. Indeed, as in the proof of Proposition 5, using the maximum principle, (106), and the easily derived bound

$$
\left|u_{\mathrm{ap}}\right| \leqq C \varepsilon^{-\frac{1}{3}}\left(3 u_{\mathrm{ap}}^{2}-a\left(\varepsilon^{\frac{2}{3}} y\right)\right), \quad y \in \mathbb{R}^{2},
$$

we can show that, if $\varepsilon$ is small, the unique solution $\varphi \in \mathscr{X} \cap C^{2+\alpha}\left(\mathbb{R}^{2}\right)$ of

$$
\mathscr{L}(\varphi)=u_{\text {ap }} f, \quad f \in \mathscr{X} \cap C^{\alpha}\left(\mathbb{R}^{2}\right), 0<\alpha<1,
$$

satisfies

$$
\|\varphi\|_{\mathscr{X}} \leqq C \varepsilon^{\frac{1}{3}}\|f\|_{\mathscr{X}}
$$


Therefore, in order to successfully apply the contraction mapping principle, as we did in the proof of Proposition 6, it is enough to construct an approximate solution $v_{\text {ap }}$ supported in a ball of radius $\mathscr{O}\left(\varepsilon^{-\frac{2}{3}}\right)$ such that

$$
\begin{aligned}
\mathscr{E} & \equiv-\Delta v_{\text {ap }}+\varepsilon^{-\frac{2}{3}} v_{\text {ap }}\left(v_{\text {ap }}^{2}-a\left(\varepsilon^{\frac{2}{3}} y\right)\right) \\
& =\mathscr{O}\left(|\ln \varepsilon|^{-\gamma} \varepsilon^{\frac{1}{3}}\right), \quad \text { uniformly in } \mathbb{R}^{2}, \text { as } \varepsilon \rightarrow 0,
\end{aligned}
$$

for some constant $\gamma>0$, and (106), (142) remain true, with $v_{\text {ap }}$ in place of $u_{\text {ap }}$, for sufficiently small $\varepsilon$ [the logarithmic term in (145) is used for convenience purposes only and has nothing to do with that appearing in (25)]. This was the main strategy followed in [191] for a related one-dimensional problem. Actually, one can plainly define an approximate solution for (51) as

$$
v_{\text {ap }}= \begin{cases}u_{\text {out }} & \text { in } \tilde{\mathscr{D}}_{\varepsilon} \backslash\left\{-2 M_{\varepsilon}<x<0\right\} \\ u_{\text {in }}+\rho_{M_{\varepsilon}}(x)\left(u_{\text {out }}-u_{\text {in }}\right) & \text { in }\left\{-2 M_{\varepsilon} \leqq x \leqq \delta \varepsilon^{-\frac{2}{3}}\right\} \\ \chi_{\delta}(x) u_{\text {in }} & \text { everywhere else }\end{cases}
$$

where $M_{\varepsilon}$ is such that $L \leqq M_{\varepsilon} \leqq \frac{\delta}{10} \varepsilon^{-\frac{2}{3}}$, and $u_{\text {in }}, u_{\text {out }}, \rho_{M}$ as in (80), (84), (100) respectively. Working as in Proposition 3, we can verify that

$$
\left|u_{\text {out }}-u_{\text {in }}\right|+|s|\left|\nabla\left(u_{\text {out }}-u_{\text {in }}\right)\right|+s^{2}\left|\Delta\left(u_{\text {out }}-u_{\text {in }}\right)\right| \leqq C\left(\varepsilon|s|^{\frac{3}{2}}+\varepsilon^{\frac{1}{3}}|s|^{-\frac{5}{2}}\right),
$$

in the region described by $\left\{-2 \delta \varepsilon^{-\frac{2}{3}} \leqq \beta s \leqq-L\right\}$. Then, as in Proposition 4 , we can show that $\mathscr{E}$, defined in (145), satisfies

$$
|\mathscr{E}| \leqq C \begin{cases}\varepsilon^{\frac{4}{3}}, & \text { in } \tilde{\mathscr{D}}_{\varepsilon} \backslash\left\{-\delta \varepsilon^{-\frac{2}{3}}<\beta s<0\right\}, \\ \varepsilon^{\frac{1}{3}}|s|^{-\frac{3}{2}}, & -\delta \varepsilon^{-\frac{2}{3}} \leqq \beta s \leqq-2 M_{\varepsilon}, \\ \varepsilon|s|^{\frac{5}{2}}+\varepsilon^{\frac{1}{3}}|s|^{-\frac{3}{2}}, & -2 M_{\varepsilon} \leqq \beta s \leqq-M_{\varepsilon}, \\ M_{\varepsilon}^{\frac{5}{2}} \varepsilon+M_{\varepsilon}^{\frac{1}{2}} \varepsilon^{\frac{5}{3}}, & -M_{\varepsilon} \leqq \beta s \leqq 0, \\ \varepsilon, & \text { everywhere else. }\end{cases}
$$

Consequently, we can achieve bound (145) by plainly choosing $M_{\varepsilon}=|\ln \varepsilon|$. Furthermore, the approximation $v_{\text {ap }}$ is sufficiently close to $u_{\text {ap }}$ so that the estimates (106) and (142) remain true with $v_{\text {ap }}$ in place of $u_{\text {ap. }}$ However, the corresponding estimates for the solution of (51), obtained using this approximation, are far from optimal. One can actually check that the above argument works because the exponent $5 / 2$ in (68) is strictly larger than one. It is worthwhile to mention that the geometric singular perturbation approach in [190] required merely (66). In $[202,203]$, for a closely related problem to $(22)_{-}$, the authors made the choice $M_{\varepsilon}=\frac{\delta}{10} \varepsilon^{-\frac{2}{3}}$ (according to our notation) while at the same time not using any convergence rate of $V(x)$ to $\sqrt{-x}$ as $x \rightarrow-\infty$, something which is not yet clear to us. 


\subsection{Proof of the Main Theorem}

We are now ready for the

PROOF OF THEOREM 1: It follows from the definition of $a_{\varepsilon}$ from (32) that

$$
\mathbf{u}_{\varepsilon}(\mathbf{y}) \equiv u_{\varepsilon}\left(\frac{\mathbf{y}}{\varepsilon^{\frac{2}{3}}}\right), \quad \mathbf{y} \in \mathbb{R}^{2},
$$

where $u_{\varepsilon}$ is the solution of (51) as in Proposition 6, is also a solution of problem (8) besides the minimizer $\eta_{\varepsilon}$ of $G_{\varepsilon}$ in $\mathscr{H}$. On the other hand, we know from Theorem 2.1 in [125] that (given $\lambda_{\varepsilon}$ ) problem (8) has a unique solution (see also Remark 16 below). Therefore, we conclude that $\mathbf{u}_{\varepsilon} \equiv \eta_{\varepsilon}$. Estimates (38)-(42) for $\eta_{\varepsilon}$ follow readily from the corresponding estimates (137)-(141) for $u_{\varepsilon}$. Relation (44) follows easily from (106) and (126).

Next, we will derive estimate (45) by building on estimates (25), (137)-(141), and using that $\left\|\eta_{\varepsilon}\right\|_{L^{2}\left(\mathbb{R}^{2}\right)}=1$. (For a self-contained proof of (8) we refer to Remark 18 below). We consider the following annular regions of the plane:

$$
\begin{aligned}
& S_{-}=\left\{-\delta \varepsilon^{-\frac{2}{3}} \leqq \beta s \leqq-2 L, 0 \leqq z \leqq \varepsilon^{-\frac{2}{3}} \ell_{\varepsilon}\right\}, \\
& S_{0}=\left\{-2 L \leqq \beta s \leqq 0,0 \leqq z \leqq \varepsilon^{-\frac{2}{3}} \ell_{\varepsilon}\right\}, \\
& S_{+}=\left\{0 \leqq \beta s \leqq \delta \varepsilon^{-\frac{2}{3}}, 0 \leqq z \leqq \varepsilon^{-\frac{2}{3}} \ell_{\varepsilon}\right\} .
\end{aligned}
$$

It follows from (101), and (127), that the solution of the stretched problem (51) satisfies

$$
u_{\varepsilon}=\tilde{u}_{\text {out }}+\mathscr{O}\left(\varepsilon|s|^{-\frac{3}{2}}\right), \quad \text { uniformly in } S_{-}, \text {as } \varepsilon \rightarrow 0 \text {. }
$$

Furthermore, from (33), (69), and (87), if $\varepsilon$ is small, we have

$$
c \varepsilon^{\frac{1}{3}}|s|^{\frac{1}{2}} \leqq \tilde{u}_{\text {out }} \leqq C \varepsilon^{\frac{1}{3}}|s|^{\frac{1}{2}} \text { in } S_{-} .
$$

So, from (9), (32), (87), and the above two relations, we find that

$$
u_{\varepsilon}^{2}-A\left(\varepsilon^{\frac{2}{3}} y\right)=\lambda_{\varepsilon}-\lambda_{0}+\varepsilon^{\frac{2}{3}} \beta^{2}\left[\beta s+V^{2}(\beta s)\right]+\mathscr{O}\left(\varepsilon^{\frac{4}{3}}|s|^{-1}\right),
$$

uniformly in $S_{-}$, as $\varepsilon \rightarrow 0$. Thus, via the identity

$$
\begin{aligned}
& \int_{S_{-}} f(y) \mathrm{d} y \\
& \quad=\int_{0}^{\varepsilon^{-\frac{2}{3}} \ell_{\varepsilon}} \int_{-\delta \varepsilon^{-\frac{2}{3}} \beta^{-1}}^{-2 L \beta^{-1}} f\left(\varepsilon^{\frac{2}{3}} s, \varepsilon^{\frac{2}{3}} z\right)\left(1+\varepsilon^{\frac{2}{3}} k_{\varepsilon}\left(\varepsilon^{\frac{2}{3}} z\right) s\right) \mathrm{d} s \mathrm{~d} z \quad \forall f \in C\left(\bar{S}_{-}\right),
\end{aligned}
$$


we obtain that

$$
\begin{aligned}
& \int_{S_{-}}\left(u_{\varepsilon}^{2}-A\left(\varepsilon^{\frac{2}{3}} y\right)\right) \mathrm{d} y=\left(\lambda_{\varepsilon}-\lambda_{0}\right) \int_{S_{-}} 1 \mathrm{~d} y \\
& \quad+\int_{0}^{\ell_{\varepsilon}} \beta_{\varepsilon}(\theta) \mathrm{d} \theta \int_{-\delta \varepsilon^{-\frac{2}{3}}}^{-2 L}\left[V^{2}(x)+x\right] \mathrm{d} x+\int_{S_{-}} \mathscr{O}\left(\varepsilon^{\frac{4}{3}}|s|^{-1}\right) \mathrm{d} s \mathrm{~d} z \\
& \stackrel{(69))}{=}\left(\lambda_{\varepsilon}-\lambda_{0}\right) \int_{S_{-}} 1 \mathrm{~d} y \\
& \quad+\int_{0}^{\ell_{\varepsilon}} \beta_{\varepsilon}(\theta) \mathrm{d} \theta \int_{-\infty}^{-2 L}\left[V^{2}(x)+x\right] \mathrm{d} x+\mathscr{O}\left(|\ln \varepsilon| \varepsilon^{\frac{2}{3}}\right),
\end{aligned}
$$

as $\varepsilon \rightarrow 0$. Similarly, recalling (36), (68), (137), and (138), we have

$$
\begin{aligned}
& \int_{S_{0}}\left(u_{\varepsilon}^{2}-A\left(\varepsilon^{\frac{2}{3}} y\right)\right) \mathrm{d} y=\left(\lambda_{\varepsilon}-\lambda_{0}\right) \int_{S_{0}} 1 \mathrm{~d} y \\
& +\int_{0}^{\ell_{\varepsilon}} \beta_{\varepsilon}(\theta) \mathrm{d} \theta \int_{-2 L}^{0}\left[V^{2}(x)+x\right] \mathrm{d} x+\mathscr{O}\left(\varepsilon^{\frac{2}{3}}\right),
\end{aligned}
$$

and

$$
\int_{S_{+}} u_{\varepsilon}^{2} \mathrm{~d} y=\int_{0}^{\ell_{\varepsilon}} \beta_{\varepsilon}(\theta) \mathrm{d} \theta \int_{0}^{\infty} V^{2}(x) \mathrm{d} x+\mathscr{O}\left(\varepsilon^{\frac{2}{3}}\right),
$$

as $\varepsilon \rightarrow 0$. Moreover, thanks to (140), (141), we have

$$
\int_{\tilde{\mathscr{D}}_{\varepsilon} \backslash\left(S_{0} \cup S_{-}\right)}\left(u_{\varepsilon}^{2}-A\left(\varepsilon^{\frac{2}{3}} y\right)\right) \mathrm{d} y=\left(\lambda_{\varepsilon}-\lambda_{0}\right) \int_{\tilde{\mathscr{D}}_{\varepsilon} \backslash\left(S_{0} \cup S_{-}\right)} 1 \mathrm{~d} y+\mathscr{O}\left(\varepsilon^{\frac{2}{3}}\right),
$$

and

$$
\int_{\mathbb{R}^{2} \backslash\left(\tilde{\mathscr{D}}_{\varepsilon} \cup S_{+}\right)} u_{\varepsilon}^{2} \mathrm{~d} y=\mathscr{O}\left(\mathrm{e}^{-c \varepsilon^{-\frac{2}{3}}}\right)
$$

as $\varepsilon \rightarrow 0$. Let us keep in mind that

$$
\int_{\mathbb{R}^{2}} u_{\varepsilon}^{2}(y) \mathrm{d} y=\varepsilon^{-\frac{4}{3}} \int_{\mathbb{R}^{2}} \eta_{\varepsilon}^{2}(\mathbf{y}) \mathrm{d} \mathbf{y}=\varepsilon^{-\frac{4}{3}} .
$$

Furthermore, recalling (6), (7), (9), and (25), for small $\varepsilon$, we can write

$$
\int_{\mathscr{D}_{\varepsilon}} A^{+}(\mathbf{y}) \mathrm{d} \mathbf{y}=\int_{\mathscr{D}_{0}} A^{+}(\mathbf{y}) \mathrm{d} \mathbf{y}-\int_{\mathscr{U}_{\varepsilon}} A^{+}(\mathbf{y}) \mathrm{d} \mathbf{y}+\int_{\mathscr{V}_{\varepsilon}} A^{+}(\mathbf{y}) \mathrm{d} \mathbf{y},
$$

where $\mathscr{U}_{\varepsilon} \subseteq \mathscr{D}_{0}, \mathscr{V}_{\varepsilon} \cap \mathscr{D}_{0}=\emptyset,\left|\mathscr{U}_{\varepsilon}\right|+\left|\mathscr{V}_{\varepsilon}\right| \leqq C|\ln \varepsilon|^{\frac{1}{2}} \varepsilon$, and $A^{+}(\mathbf{y}) \leqq C|\ln \varepsilon|^{\frac{1}{2}} \varepsilon$ if $\mathbf{y} \in \mathscr{U}_{\varepsilon} \cup \mathscr{V}_{\varepsilon}$. Thus, via (5), (52), we infer that

$$
\int_{\tilde{\mathscr{D}}_{\varepsilon}} A^{+}\left(\varepsilon^{\frac{2}{3}} y\right) \mathrm{d} y=\varepsilon^{-\frac{4}{3}}+\mathscr{O}\left(|\ln \varepsilon| \varepsilon^{\frac{2}{3}}\right) \quad \text { as } \varepsilon \rightarrow 0 .
$$


By combining (148)-(154), we deduce that

$$
\begin{aligned}
& \left(\lambda_{\varepsilon}-\lambda_{0}\right)\left|\tilde{\mathscr{D}}_{\varepsilon}\right|+\left(\int_{0}^{\ell_{\varepsilon}} \beta_{\varepsilon}(\theta) \mathrm{d} \theta\right)\left(\int_{-\infty}^{0}\left[V^{2}(x)+x\right] \mathrm{d} x+\int_{0}^{\infty} V^{2}(x) \mathrm{d} x\right) \\
& =\mathscr{O}\left(|\ln \varepsilon| \varepsilon^{\frac{2}{3}}\right)
\end{aligned}
$$

as $\varepsilon \rightarrow 0$. Now, the validity of estimate (45) follows readily by noting that the sum of the above two integral involving $V$ is zero. This can be seen by multiplying (65) by $V_{x}$, integrating the resulting identity by parts over $(-\infty, 0)$ and $(0, \infty)$ respectively, and recalling (68).

To finish, utilizing all the above, we will establish the validity of estimate (46) for the energy of $\eta_{\varepsilon}$. It is straightforward to see that

$$
G_{\varepsilon}\left(\eta_{\varepsilon}\right)=\int_{\mathbb{R}^{2}}\left\{\frac{1}{2}|\nabla u|^{2}+\frac{\varepsilon^{-\frac{2}{3}}}{4} u^{4}+\frac{\varepsilon^{-\frac{2}{3}}}{2} W\left(\varepsilon^{\frac{2}{3}} y\right) u^{2}\right\} \mathrm{d} y,
$$

where $u(y)=\eta_{\varepsilon}\left(\varepsilon^{\frac{2}{3}} y\right)$ is the solutions of the stretched problem (51). Motivated from (10), and recalling (5), it is easy to check that we can rewrite the above relation as

$$
\begin{aligned}
G_{\varepsilon}\left(\eta_{\varepsilon}\right)= & \frac{1}{2} \int_{\mathbb{R}^{2}}|\nabla u|^{2} \mathrm{~d} y+\frac{\varepsilon^{-\frac{2}{3}}}{4} \int_{\tilde{\mathscr{D}}_{\varepsilon}}\left(u^{2}-a\left(\varepsilon^{\frac{2}{3}} y\right)\right)^{2} \mathrm{~d} y \\
& -\frac{1}{4}\left(\int_{\mathbb{R}^{2}}\left(A^{+}\right)^{2} \mathrm{~d} \mathbf{y}\right) \varepsilon^{-2}+\frac{\lambda_{0}}{2} \varepsilon^{-2} \\
& -\frac{\left(\lambda_{\varepsilon}-\lambda_{0}\right)^{2}}{4}\left|\mathscr{D}_{0}\right| \varepsilon^{-2}+\frac{\varepsilon^{-\frac{2}{3}}}{4} \int_{\mathbb{R}^{2} \backslash \tilde{\mathscr{D}}_{\varepsilon}}\left(u^{2}-a\left(\varepsilon^{\frac{2}{3}} y\right)\right)^{2} \mathrm{~d} y \\
& -\frac{\varepsilon^{-\frac{2}{3}}}{4} \int_{\mathbb{R}^{2} \backslash\left(\varepsilon^{-\frac{2}{3}} \mathscr{D}_{0}\right)} a^{2}\left(\varepsilon^{\frac{2}{3}} y\right) \mathrm{d} y .
\end{aligned}
$$

Similarly to the above proof of (45), keeping in mind the proof of Proposition 6, (45), (68), (97), (99), (137), and relations (161), (165) below [whose proofs do not require (46)], we get

$$
\begin{aligned}
\int_{\mathbb{R}^{2}}|\nabla u|^{2} \mathrm{~d} y & =\int_{S_{-}}\left(\varepsilon^{\frac{2}{3}} \beta^{4} V_{x}^{2}(\beta s)+\mathscr{O}\left(\varepsilon^{\frac{4}{3}}\right)\right)\left(1+\varepsilon^{\frac{2}{3}} k s\right) \mathrm{d} s \mathrm{~d} z+\mathscr{O}(1) \\
& =\left(\int_{0}^{\ell_{\varepsilon}} \beta_{\varepsilon}^{3}(\theta) \mathrm{d} \theta\right) \int_{-\delta \varepsilon^{-\frac{2}{3}}}^{-2 L} V_{x}^{2}(x) \mathrm{d} x+\mathscr{O}(1) \\
& =\frac{1}{6}\left(\int_{0}^{\ell_{0}} \beta_{0}^{3}(\theta) \mathrm{d} \theta\right)|\ln \varepsilon|+\mathscr{O}(1),
\end{aligned}
$$

as $\varepsilon \rightarrow 0$. Furthermore, as in the above proof of (45), we have

$$
\frac{\varepsilon^{-\frac{2}{3}}}{4} \int_{\tilde{\mathscr{D}}_{\varepsilon}}\left(u^{2}-a\left(\varepsilon^{\frac{2}{3}} y\right)\right)^{2} \mathrm{~d} y=\mathscr{O}(1)
$$


and

$$
\frac{\varepsilon^{-\frac{2}{3}}}{4} \int_{\mathbb{R}^{2} \backslash \tilde{\mathscr{D}}_{\varepsilon}}\left(u^{2}-a\left(\varepsilon^{\frac{2}{3}} y\right)\right)^{2} \mathrm{~d} y-\frac{\varepsilon^{-\frac{2}{3}}}{4} \int_{\mathbb{R}^{2} \backslash\left(\varepsilon^{-\frac{2}{3}} \mathscr{D}_{0}\right)} a^{2}\left(\varepsilon^{\frac{2}{3}} y\right) \mathrm{d} y=\mathscr{O}\left(\varepsilon^{\frac{2}{3}}\right),
$$

as $\varepsilon \rightarrow 0$. Now, the validity of (46) follows at once from (45), (155), and the above three relations. Alternatively, we could have used the formula

$$
G_{\varepsilon}\left(\eta_{\varepsilon}\right)=\frac{1}{2 \varepsilon^{2}} \lambda_{\varepsilon}-\frac{1}{4 \varepsilon^{2}} \int_{\mathbb{R}^{2}} \eta_{\varepsilon}^{4} \mathrm{~d} \mathbf{y},
$$

which follows easily by testing Equation (8) with $\eta_{\varepsilon}$.

The proof of Theorem 1 is complete.

We now outline a few remarks.

Remark 13. The maximum principle yields the upper bound:

$$
\eta_{\varepsilon}(\mathbf{y}) \leqq \max _{\mathbb{R}^{2}} \sqrt{a_{\varepsilon}^{+}}, \quad \mathbf{y} \in \mathbb{R}^{2}
$$

[see also (156) below].

Remark 14. As in [125], where the authors refer to an idea of Shafrir, we can rewrite (8) in the form

$$
-\varepsilon^{2} \Delta\left(\sqrt{a_{\varepsilon}}-\eta_{\varepsilon}\right)+\eta_{\varepsilon}\left(\eta_{\varepsilon}+\sqrt{a_{\varepsilon}}\right)\left(\eta_{\varepsilon}-\sqrt{a_{\varepsilon}}\right)=-\varepsilon^{2} \Delta\left(\sqrt{a_{\varepsilon}}\right) \text { in } \mathscr{D}_{\varepsilon} .
$$

The above relation suggests the following, which can be proven similar to [150]: We have

$$
\eta_{\varepsilon}(\mathbf{y})=\sqrt{a_{\varepsilon}}+\varepsilon^{2} \frac{\Delta\left(\sqrt{a_{\varepsilon}}\right)}{2 a_{\varepsilon}}+o\left(\varepsilon^{2}\right)
$$

where $\varepsilon^{-2} o\left(\varepsilon^{2}\right) \rightarrow 0$ uniformly on any compact subset of $\mathscr{D}_{0}$ as $\varepsilon \rightarrow 0$. Keeping in mind (7) which implies that

$$
\varepsilon^{2} \frac{\Delta\left(\sqrt{a_{\varepsilon}}\right)}{2 a_{\varepsilon}}=\mathscr{O}\left(\varepsilon^{2}|t|^{-\frac{5}{2}}\right) \text { uniformly in } \mathscr{D}_{0} \text { as } \varepsilon \rightarrow 0,
$$

and (40), we are tempted to believe that (156) can be extended to hold uniformly in the domain $\mathscr{D}_{\varepsilon} \backslash\left\{-K \varepsilon^{\frac{2}{3}}<t<0\right\}$, with $K$ large, if $\varepsilon \rightarrow 0$. A possible approach could be by seeking a more refined inner solution with $V+\varepsilon \phi$ in place of $V$ in (80), where $\phi$ is determined by solving a linear equation of the form $\mathscr{M}(\phi)=$ $f(x, z), x \in \mathbb{R}, z \in\left[0, \varepsilon^{-\frac{2}{3}} \ell_{\varepsilon}\right)$ with $f$ known (in terms of the curvature $k_{\varepsilon}$, $V, a_{\varepsilon}$ and their derivatives) and $\mathscr{M}$ as in (70); we refer to [135] for a related problem. Actually, we have computed that in the radially symmetric case, in $N \geqq 1$ dimensions, we have

$$
\mathscr{M}(\phi)=\frac{N-1}{R_{\varepsilon}} \beta^{-1} V_{x}-\frac{1}{2} a_{r r}\left(R_{\varepsilon}\right) \beta^{-4} x^{2} V,
$$


where $R_{\varepsilon}$ is the radius of $\mathscr{D}_{\varepsilon}$ and $\beta=\left[-a_{r}\left(R_{\varepsilon}\right)\right]^{\frac{1}{3}}$. Moreover, due to matching conditions with $a_{\varepsilon}\left(R_{\varepsilon}+\varepsilon^{\frac{2}{3}} \beta^{-1} x\right)$, we need that

$$
\phi(x)+\frac{1}{4} a_{r r}\left(R_{\varepsilon}\right) \beta^{-4}(-x)^{\frac{3}{2}} \rightarrow 0, \quad x \rightarrow-\infty ; \phi \rightarrow 0, x \rightarrow \infty .
$$

In the special case of the model harmonic potential, this lower order term in the inner solution has been formally derived in [94] and rigorously in [104]. It might also be useful for the reader to take a look at (181) below.

Remark 15. As in [125, Prop. 2.1 e)], it follows that

$$
\left\|\eta_{\varepsilon}-\sqrt{a_{\varepsilon}}\right\|_{C^{1}(\mathscr{K})} \leqq C \mathscr{K} \varepsilon^{2} \text { for any compact subset } \mathscr{K} \subset \mathscr{D}_{0} \text {, }
$$

if $\varepsilon$ is small.

Remark 16. Given $\lambda>\min _{\mathbb{R}^{N}} W$, problem (22) , in $N=2$ dimensions, has a unique positive solution, for small $\varepsilon$, as has been proven recently in [125] by combining ideas of BrezIs and Oswald [43] (see also the uniqueness part of Proposition 11 herein) with those used in the proof of De Giorgi's conjecture in low dimensions [107]. This fact allows us to work exclusively with Equation (8), since positive solutions of the latter coincide with the unique real valued minimizer of $G_{\varepsilon}$ in $\mathscr{H}$. On the other hand, using (27), which holds for every positive solution of $(22)_{-}$, it is easy to see that the method of [125] can be extended to the case of arbitrary $N \geqq 1$ dimensions. Hence, our Theorem 1 can be extended naturally to treat the case where the functional $G_{\varepsilon}$ is considered in arbitrary dimensions, with the analogous conditions on the potential. In the radially symmetric case, uniqueness results which allow the case where $\lambda=\inf _{\mathbb{R}^{N}} W$ may be found in [3] and [104].

Remark 17. One can also prove an analogous result to Theorem 1 for the real valued minimizer of $G_{\varepsilon}$ in

$$
\mathscr{J} \equiv\left\{u \in W_{0}^{1,2}\left(\mathscr{D}_{0} ; \mathbb{C}\right): \int_{\mathscr{D}_{0}}|u|^{2} \mathrm{~d} y=1\right\} .
$$

This problem has been studied in [130], in a three-dimensional setting, with potentials of the form (16). The special property that $\Delta W>0$ in $\mathscr{D}_{0}$ was used in an essential way in the latter reference for estimating the minimizer near the surface $\partial \mathscr{D}_{0}$, along which it has a steep corner layer. We also refer the interested reader to [8] and [13] for the case where $\mathscr{D}_{0}$ has annular shape. For a numerical treatment of the problem we refer to [29]. As in Section 3.1 below, it is not hard to see that in this case the layer profile near $\partial \mathscr{D}_{0}$ should be determined by the unique solution of

$$
\begin{cases}v_{x x}-v\left(v^{2}+x\right)=0, & x<0, \\ v(x)-\sqrt{-x} \rightarrow 0 \text { as } x \rightarrow-\infty ; & v(0)=0 .\end{cases}
$$

We refer to Appendix B below for a treatment of the above problem in relation with (37).

The minimization of the functional $G_{\varepsilon}$ in $W^{1,2}\left(\mathscr{D}_{0}\right)$, subject to the mass constraint, leads to the equation in (22) _ with Neumann boundary conditions. The latter singular perturbation problem may be treated by using in place of $V$, in (80), the (reflection of the) solution described in Remark 36 below. 
Remark 18. Identical estimates to (38)-(42) (with $t$ replaced by the signed distance from $\partial_{0}$ ) hold for the solution $\tilde{\eta}_{\varepsilon}$ of (22)_ (with $\lambda=\lambda_{0}, q=3$ ). One can use the function $\frac{\tilde{\eta}_{\varepsilon}}{\left\|\tilde{\eta}_{\varepsilon}\right\|_{L^{2}\left(\mathbb{R}^{2}\right)}} \in \mathscr{H}$ as a competitor in order to give a self-contained proof of (11), from which (25) follows readily (see [10,125]). (As in the proof of (46), keeping in mind that $\left\|\tilde{\eta}_{\varepsilon}\right\|_{L^{2}\left(\mathbb{R}^{2}\right)}^{2} \rightarrow\left\|A^{+}\right\|_{L^{1}\left(\mathbb{R}^{2}\right)}$, the main contribution would be from the gradient term).

Remark 19. In the case where the potential $W$ is of harmonic type, as in (16), it was observed by the authors of [125] that

$$
\eta_{\varepsilon}(\mathbf{y})=\frac{\sqrt{\lambda_{0}+\lambda_{\varepsilon}}}{\sqrt{\lambda_{0}}} \tilde{\eta}_{\tilde{\varepsilon}}\left(\frac{\sqrt{\lambda_{0}} \mathbf{y}}{\sqrt{\lambda_{0}+\lambda_{\varepsilon}}}\right) \quad \text { with } \tilde{\varepsilon}=\frac{\lambda_{0} \varepsilon}{\lambda_{0}+\lambda_{\varepsilon}}
$$

where $\tilde{\eta}_{\tilde{\varepsilon}}$ was defined in Remark 18 . This identity and a technique of Struwe [125, Lemma 2.3] were used essentially in their proof of (45) for this special class of potentials.

Remark 20. Relation (44) implies that, if $\varepsilon$ is small, the linearized operator $\mathscr{L}_{\varepsilon}$ about $u_{\varepsilon}$ is invertible. Hence, the implicit function theorem (see for instance [19]) implies that there exists a small number $\varepsilon_{0}>0$ such that, in addition to being isolated (for each $\varepsilon$ ), the minimizers $\eta(\varepsilon) \equiv \eta_{\varepsilon}$ depend smoothly on $\varepsilon \in\left(0, \varepsilon_{0}\right)$ (in all the usual function spaces). In particular, we have $\eta:\left(0, \varepsilon_{0}\right) \rightarrow W^{1,2}\left(\mathbb{R}^{2}\right)$ is $C^{1}$. This last property yields at once the first part of Lemma 2.3 in [125], mentioned in Remark 19 above.

Remark 21. In the special case of the model harmonic potential $W(\mathbf{y})=|\mathbf{y}|^{2}$, since $\eta_{\varepsilon}$ is radially symmetric [108], we can define $v_{\varepsilon}:\left(-\infty, \lambda_{\varepsilon} \varepsilon^{-\frac{2}{3}}\right] \rightarrow \mathbb{R}$ by

$$
\eta_{\varepsilon}(\mathbf{y})=\varepsilon^{\frac{1}{3}} v_{\varepsilon}\left(\frac{\lambda_{\varepsilon}-|\mathbf{y}|^{2}}{\varepsilon^{\frac{2}{3}}}\right), \quad \mathbf{y} \in \mathbb{R}^{2}
$$

Letting $\xi=\left(\lambda_{\varepsilon}-|\mathbf{y}|^{2}\right) / \varepsilon^{\frac{2}{3}}$, then the equation in (8) becomes equivalent to

$$
4\left(\lambda_{\varepsilon}-\varepsilon^{\frac{2}{3}} \xi\right) \partial_{\xi \xi} v_{\varepsilon}-4 \varepsilon^{\frac{2}{3}} \partial_{\xi} v_{\varepsilon}+\xi v_{\varepsilon}-v_{\varepsilon}^{3}=0, \quad \xi \in\left(-\infty, \lambda_{\varepsilon} \varepsilon^{-\frac{2}{3}}\right]
$$

At first glance this might look rather counterintuitive but this strategy, already used in [104], allows one to use directly $\left(2 \lambda_{\varepsilon}\right)^{\frac{1}{3}} V\left(-\frac{\xi}{\left(2 \lambda_{\varepsilon}\right)^{\frac{2}{3}}}\right)$ as a global approximation [recall (65)-(69)].

Remark 22. In the radially symmetric case, estimate (46) was formally predicted in $[67,68]$, and rigorously proven and extended very recently, for the case of the harmonic potential, in [105] at the same time that the current paper was written. 


\section{Further Properties of the Ground State $\eta_{\varepsilon}$}

As a byproduct of our construction of the ground state $\eta_{\varepsilon}$, we can extend to the non-radial case relation (30), improve relation (29), show that $\eta_{\varepsilon}$ has maximal Hölder regularity, and improve relation (28). Finally, under an additional but natural non-degeneracy assumption on the potential, we can refine bound (39).

Corollary 2. There exist small constants $c, d^{\prime}>0$ such that, given $D>0$, we have

$$
\left(\eta_{\varepsilon}\right)_{t} \leqq-c\left(|t|+\varepsilon^{\frac{2}{3}}\right)^{-\frac{1}{2}}
$$

in $\left\{-d^{\prime} \leqq t \leqq D \varepsilon^{\frac{2}{3}}, \theta \in\left[0, \ell_{\varepsilon}\right)\right\}$, provided $\varepsilon$ is sufficiently small.

Proof. We will present it for the solution $u_{\varepsilon}$ of the stretched problem (51). It follows from (129) that, if $\varepsilon$ is small, the function $\varphi_{*}=u_{\varepsilon}-u_{\text {ap }}$ satisfies

$$
\left|\Delta_{y} \varphi_{*}\right| \leqq \begin{cases}C \varepsilon(|s|+1)^{-\frac{1}{2}} & \text { if }-10 \delta \varepsilon^{-\frac{2}{3}} \leqq \beta s \leqq 0 \\ C \varepsilon \mathrm{e}^{-c|s|} & \text { if } 0 \leqq \beta s \leqq 10 \delta \varepsilon^{-\frac{2}{3}}\end{cases}
$$

In establishing the above estimate, the term that needed extra care was

$$
\varepsilon^{-\frac{2}{3}}\left(3 u_{\mathrm{ap}}^{2}-a\left(\varepsilon^{\frac{2}{3}} y\right)\right) \varphi_{*}
$$

which can be estimated by noting that

$$
\begin{aligned}
& u_{\text {ap }}^{2}+\left|a\left(\varepsilon^{\frac{2}{3}} y\right)\right| \leqq C \varepsilon^{\frac{2}{3}}(|s|+1) \text { and }\left|\varphi_{*}\right| \leqq C \varepsilon(|s|+1)^{-\frac{1}{2}} \quad \text { if } \\
& \left\{|\beta s| \leqq 10 \delta \varepsilon^{-\frac{2}{3}}\right\},
\end{aligned}
$$

[recall (33), (107), (108), and (127)]. Making (mild) use of (159) and the second estimate in (160), via standard interior elliptic regularity estimates [109] (applied on balls of radius one) or the interpolation-type inequality of Lemma A.1 in [38], the statement of which is included below as Lemma 1 for the reader's convenience, we obtain that

$$
\left|\nabla_{y} \varphi_{*}\right| \leqq C \varepsilon
$$

in the neighborhood of $\tilde{\Gamma}_{\varepsilon}$ described by $\left\{|\beta s| \leqq 5 \delta \varepsilon^{-\frac{2}{3}}\right\}$, provided $\varepsilon$ is sufficiently small.

By (67), (80), (99), (101), and (161), we deduce that, given $\tilde{D}>0$,

$$
\left(u_{\varepsilon}\right)_{s}=\varepsilon^{\frac{1}{3}} \beta^{2} V_{x}(\beta s)+\mathscr{O}(\varepsilon)<-c \varepsilon^{\frac{1}{3}},
$$

uniformly in the neighborhood of $\tilde{\Gamma}_{\varepsilon}$ described by $\{-L \leqq x \leqq \tilde{D}\}$, as $\varepsilon \rightarrow 0$. In the same manner, recalling (69) and the second estimate in (90), we find that

$$
\begin{aligned}
\left(u_{\varepsilon}\right)_{s} & =\varepsilon^{\frac{1}{3}} \beta^{2} V_{x}(\beta s)+\mathscr{O}\left(\varepsilon|s|^{\frac{1}{2}}\right) \\
& \leqq-c \varepsilon^{\frac{1}{3}}|s|^{-\frac{1}{2}}+C \varepsilon|s|^{\frac{1}{2}} \\
& \leqq-\frac{c}{2} \varepsilon^{\frac{1}{3}}|s|^{-\frac{1}{2}},
\end{aligned}
$$


as long as $-\tilde{d} \varepsilon^{-\frac{2}{3}}<\beta s<-L$, for some small constant $\tilde{d}$, provided $\varepsilon$ is sufficiently small. The corresponding assertion of the corollary, for the solution of the equivalent stretched problem (51), follows readily from (162) and (163).

The proof of the corollary is complete.

The following is Lemma A.1 in [38]:

Lemma 1. Assume that $u$ satisfies

$$
-\Delta u=f \quad \text { in } \Omega \subset \mathbb{R}^{N} .
$$

Then

$$
|\nabla u(\boldsymbol{y})|^{2} \leqq C\left\{\|f\|_{L^{\infty}(\Omega)}\|u\|_{L^{\infty}(\Omega)}+\frac{1}{\operatorname{dist}^{2}(\boldsymbol{y}, \partial \Omega)}\|u\|_{L^{\infty}(\Omega)}^{2}\right\} \quad \forall \boldsymbol{y} \in \Omega,
$$

where $C$ is some constant depending only on $N$.

We can also improve the bound (29):

Corollary 3. If $\varepsilon>0$ is sufficiently small, we have

$$
\left\|\nabla \eta_{\varepsilon}\right\|_{L^{\infty}\left(\mathbb{R}^{2}\right)} \leqq C \varepsilon^{-\frac{1}{3}}
$$

Proof. We will prove the corresponding assertion for the solution $u_{\varepsilon}$ of the stretched problem (51). From the proof of Proposition 6, recalling (69), (97), (98), (99), and (161), we find that

$$
|\nabla u(y)| \leqq C \varepsilon^{\frac{1}{3}} \quad \text { if } y \in\left\{|x| \leqq 3 \delta \varepsilon^{-\frac{2}{3}}, z \in\left[0, \varepsilon^{-\frac{2}{3}} \ell_{\varepsilon}\right)\right\},
$$

if $\varepsilon$ is small. Furthermore, recalling (127), we get

$$
u^{2}-a\left(\varepsilon^{\frac{2}{3}} y\right)=2 \varphi_{*} \sqrt{a\left(\varepsilon^{\frac{2}{3}} y\right)}+\varphi_{*}^{2}=\mathscr{O}\left(\varepsilon^{2}\right),
$$

uniformly in $\tilde{\mathscr{D}}_{\varepsilon} \backslash\left\{-2 \delta \varepsilon^{-\frac{2}{3}} \leqq x \leqq 0\right\}$, as $\varepsilon \rightarrow 0$. Hence, by the equation (51), we obtain that

$$
|\Delta u| \leqq C \varepsilon^{\frac{4}{3}} \text { in } \tilde{\mathscr{D}}_{\varepsilon} \backslash\left\{-2 \delta \varepsilon^{-\frac{2}{3}} \leqq x \leqq 0\right\}
$$

Consequently, by the interpolation-type inequality of Lemma A.1 in [38] (see Lemma 1 above), we infer that

$$
|\nabla u| \leqq C \varepsilon^{\frac{2}{3}} \text { in } \tilde{\mathscr{D}}_{\varepsilon} \backslash\left\{-3 \delta \varepsilon^{-\frac{2}{3}} \leqq x \leqq 0\right\}
$$

Similarly, recalling (4) and (41), we have

$$
|\nabla u| \leqq C e^{-c \varepsilon^{-\frac{2}{3}}} \quad \text { outside of } \tilde{\mathscr{D}}_{\varepsilon} \cup\left\{0 \leqq x \leqq 3 \delta \varepsilon^{-\frac{2}{3}}\right\} .
$$

The corresponding assertion of the corollary for $u$ follows readily from (164)-(166).

The proof of the corollary is complete. 
In the following corollary, we will show that $\eta_{\varepsilon}$ has the maximal Hölder regularity available (recall Definition 1 from Section 1.2).

Corollary 4. If $\varepsilon$ is sufficiently small, we have

$$
\left\|\eta_{\varepsilon}\right\|_{C^{1 / 2}\left(\mathbb{R}^{2}\right)} \leqq C .
$$

Proof. From (38), (39), (68), (69), (99), and (161), abusing notation, it follows that

$$
\left\{\begin{array}{l}
c\left(|t|+\varepsilon^{\frac{2}{3}}\right)^{\frac{1}{2}} \leqq \eta_{\varepsilon} \leqq C\left(|t|+\varepsilon^{\frac{2}{3}}\right)^{\frac{1}{2}} \\
\left|\left(\eta_{\varepsilon}\right)_{t}\right| \leqq C\left(|t|+\varepsilon^{\frac{2}{3}}\right)^{-\frac{1}{2}} \\
\left|\left(\eta_{\varepsilon}\right)_{\theta}\right| \leqq C\left(|t|+\varepsilon^{\frac{2}{3}}\right)^{\frac{1}{2}}
\end{array}\right.
$$

in the region described by $\{|t| \leqq 3 \delta\}$, if $\varepsilon$ is small, having further decreased the value of $\delta$ if necessary. Abusing notation once more, let $\mathbf{y}_{i}=\left(t_{i}, \theta_{i}\right), i=1$, 2, with $\left|t_{i}\right| \leqq 3 \delta$ and $\theta_{i} \in\left[0, \ell_{\varepsilon}\right)$, be any two points in that region. We write

$$
\eta_{\varepsilon}\left(\mathbf{y}_{1}\right)-\eta_{\varepsilon}\left(\mathbf{y}_{2}\right)=\eta_{\varepsilon}\left(t_{1}, \theta_{1}\right)-\eta_{\varepsilon}\left(t_{2}, \theta_{1}\right)+\eta_{\varepsilon}\left(t_{2}, \theta_{1}\right)-\eta_{\varepsilon}\left(t_{2}, \theta_{2}\right) .
$$

Now, instead of considering the difference $\eta_{\varepsilon}\left(t_{1}, \theta_{1}\right)-\eta_{\varepsilon}\left(t_{2}, \theta_{1}\right)$, we will first consider the difference $\eta_{\varepsilon}^{2}\left(t_{1}, \theta_{1}\right)-\eta_{\varepsilon}^{2}\left(t_{2}, \theta_{1}\right)$. We have

$$
\eta_{\varepsilon}^{2}\left(t_{1}, \theta_{1}\right)-\eta_{\varepsilon}^{2}\left(t_{2}, \theta_{1}\right)=\left(t_{1}-t_{2}\right) \int_{0}^{1} 2 \eta \eta_{t}\left(t_{1}+r\left(t_{2}-t_{1}\right), \theta_{1}\right) \mathrm{d} r .
$$

So, thanks to (168), we find that

$$
\left|\eta_{\varepsilon}^{2}\left(t_{1}, \theta_{1}\right)-\eta_{\varepsilon}^{2}\left(t_{2}, \theta_{1}\right)\right| \leqq C\left|t_{1}-t_{2}\right| .
$$

In turn, via the lower bound in (168), the above relation yields that

$$
\begin{aligned}
\left|\eta_{\varepsilon}\left(t_{1}, \theta_{1}\right)-\eta_{\varepsilon}\left(t_{2}, \theta_{1}\right)\right| & \leqq C \frac{\left|t_{1}-t_{2}\right|^{\frac{1}{2}}}{\left|t_{1}\right|^{\frac{1}{2}}+\left|t_{2}\right|^{\frac{1}{2}}}\left|t_{1}-t_{2}\right|^{\frac{1}{2}} \\
& \leqq C\left(\left|t_{1}-t_{2}\right|+\left|\theta_{1}-\theta_{2}\right|\right)^{\frac{1}{2}}
\end{aligned}
$$

Similarly, we obtain that

$$
\left|\eta_{\varepsilon}^{2}\left(t_{2}, \theta_{1}\right)-\eta_{\varepsilon}^{2}\left(t_{2}, \theta_{2}\right)\right| \leqq C\left(\left|t_{2}\right|+\varepsilon^{\frac{2}{3}}\right)\left|\theta_{1}-\theta_{2}\right|,
$$

which, as before, implies that

$$
\begin{aligned}
\left|\eta_{\varepsilon}\left(t_{2}, \theta_{1}\right)-\eta_{\varepsilon}\left(t_{2}, \theta_{2}\right)\right| & \leqq C\left(\left|t_{2}\right|+\varepsilon^{\frac{2}{3}}\right)^{\frac{1}{2}}\left|\theta_{1}-\theta_{2}\right| \\
& \leqq C\left(\left|t_{1}-t_{2}\right|+\left|\theta_{1}-\theta_{2}\right|\right)^{\frac{1}{2}} .
\end{aligned}
$$


Hence, by (169), (170), and (171) [also keeping in mind Remark 13], we deduce that

$$
\left\|\eta_{\varepsilon}\right\|_{C^{1 / 2}(|t| \leqq 3 \delta)} \leqq C,
$$

if $\varepsilon$ is small. That was the hard part. In the remaining regions of the plane, by virtue of (165) and (166), we see that $\left|\nabla \eta_{\varepsilon}\right| \leqq C$ which implies that, in those regions, the family $\eta_{\varepsilon}$ is in fact uniformly Lipschitz continuous, as $\varepsilon \rightarrow 0$.

The proof of the corollary is complete.

Remark 23. Uniform Hölder $C^{0, \alpha}, 0<\alpha<1$, bounds for Gross-Pitaevskii systems, where the singular limit functions have Lipschitz regularity $(\alpha=1)$, have been proven in [167] by blow-up techniques and the monotonicity formulae of Almgren and Alt, Caffarelli, and Friedman (see also [54]). The importance of our result lies in the fact that the Hölder exponent $1 / 2$ in (167) equals to the exact maximal Hölder regularity of the singular limit profile. To the best of our knowledge, this property has been proven, in singular perturbation problems, only in one-dimensional problems, see [36].

The following corollary answers a question posed to one of us by A. Tertikas in relation with [191].

Corollary 5. Given $\alpha \in\left[0, \frac{1}{2}\right)$, we have

$$
\eta_{\varepsilon} \rightarrow \sqrt{A^{+}} \text {in } C^{\alpha}\left(\mathbb{R}^{2}\right) \text { as } \varepsilon \rightarrow 0
$$

but $\eta_{\varepsilon}$ does not converge to $\sqrt{A^{+}}$in $C^{\frac{1}{2}}\left(\mathbb{R}^{2}\right)$ as $\varepsilon \rightarrow 0$.

Proof. Given $\alpha \in\left[0, \frac{1}{2}\right.$ ), in view of (13), Corollary 4, and the compactness of the embedding $C^{\frac{1}{2}}\left(2 \overline{\mathscr{D}}_{0}\right) \hookrightarrow C^{\alpha}\left(2 \overline{\mathscr{D}}_{0}\right)$ (see [109]), we find that $\eta_{\varepsilon} \rightarrow \sqrt{A^{+}}$in $C^{\alpha}\left(2 \overline{\mathscr{D}}_{0}\right)$ as $\varepsilon \rightarrow 0$. Now, the desired relation (172) follows via (42) and (166).

On the other hand, the following simple argument shows that we do not have convergence in $C^{\frac{1}{2}}$. Let $\left\{\varepsilon_{n}\right\}$ be a decreasing sequence such that $\varepsilon_{n} \rightarrow 0$, and, abusing notation, consider the points $\mathbf{y}_{n}=\left(t_{n}, \theta_{n}\right)=\left(-\varepsilon_{n}^{\frac{2}{3}}, 0\right)$. If $n<m$, thanks to (36), (38), and (161), we get

$$
\left\|\eta_{\varepsilon_{n}}-\eta_{\varepsilon_{m}}\right\|_{C^{\frac{1}{2}\left(\mathbb{R}^{2}\right)}} \geqq \frac{\left|\eta_{\varepsilon_{n}}\left(\mathbf{y}_{n}\right)-\eta_{\varepsilon_{m}}\left(\mathbf{y}_{m}\right)\right|}{\left|\mathbf{y}_{n}-\mathbf{y}_{m}\right|^{\frac{1}{2}}} \geqq c\left(\frac{\varepsilon_{n}^{\frac{1}{3}}-\varepsilon_{m}^{\frac{1}{3}}}{\varepsilon_{n}^{\frac{1}{3}}+\varepsilon_{m}^{\frac{1}{3}}}\right)^{\frac{1}{2}}-C \varepsilon_{n}^{\frac{2}{3}},
$$

where $c, C$ are independent of $n, m$. Now, choosing for example $\varepsilon_{n}=\left(\frac{1}{n}\right)^{3}$ and $m=2 n$, we conclude that $\left\{\eta_{\varepsilon_{n}}\right\}$ is not Cauchy in $C^{\frac{1}{2}}$.

The proof of the corollary is complete.

Remark 24. We expect that there exists some constant $C>0$ such that, for small $\varepsilon>0$, we have

$$
\left\|\eta_{\varepsilon}-\sqrt{A^{+}}\right\|_{C^{\alpha}\left(\mathbb{R}^{2}\right)} \leqq C \varepsilon^{\frac{2}{3}\left(\frac{1}{2}-\alpha\right)}, \quad 0 \leqq \alpha \leqq \frac{1}{2},
$$

see also Remark 26 below. 
The following result is motivated from (28):

Corollary 6. Given $0<\alpha \leqq 1$, if $\varepsilon$ is sufficiently small, and $A$ as in (9), there exists a constant $C>0$ such that

$$
\left|\eta_{\varepsilon}-\sqrt{A^{+}}\right| \leqq C\left(\varepsilon^{2-\frac{5}{3} \alpha}+\varepsilon^{-\frac{1}{3} \alpha}\left|\lambda_{\varepsilon}-\lambda_{0}\right|\right) \leqq C\left(\varepsilon^{2-2 \alpha}+\varepsilon^{\frac{4}{3}-\frac{2}{3} \alpha}\right) \sqrt{A^{+}},
$$

at points in $\mathscr{D}_{0}$ whose distance from $\partial \mathscr{D}_{0}$ is greater than $\varepsilon^{\frac{2}{3} \alpha}$.

Proof. From (6), (7), (9), (25), and (40), it follows that in the region $\mathscr{D}_{0} \backslash\left\{-\frac{1}{2} \varepsilon^{\frac{2}{3} \alpha}<\right.$ $t<0\}$ we have:

$$
\begin{aligned}
& \sqrt{A(\mathbf{y})} \geqq c \varepsilon^{\frac{\alpha}{3}}, \\
& \sqrt{A(\mathbf{y})}-\sqrt{\lambda_{\varepsilon}-W(\mathbf{y})}=\mathscr{O}\left(\left|\lambda_{\varepsilon}-\lambda_{0}\right| \varepsilon^{-\frac{\alpha}{3}}\right),
\end{aligned}
$$

and

$$
\eta_{\varepsilon}(\mathbf{y})-\sqrt{\lambda_{\varepsilon}-W(\mathbf{y})}=\mathscr{O}\left(\varepsilon^{2-\frac{5}{3} \alpha}\right),
$$

uniformly, as $\varepsilon \rightarrow 0$. Now, the assertion of the corollary follows readily by combining (45), Remark 3, and the above three relations.

The proof is complete.

Remark 25. Note that estimate (173), when $\alpha=\frac{1}{2}$, considerably improves estimate (28), which was originally proven in [8] (for solutions of (8) with $\lambda_{\varepsilon}=\lambda_{0}$ ) and in the sequel used in $[10,13]$, and [125].

Remark 26. By (38)-(39), and (173) with $\alpha=1$, for small $\varepsilon>0$, we obtain that

$$
\left\|\eta_{\varepsilon}-\sqrt{A^{+}}\right\|_{L^{\infty}\left(\mathbb{R}^{2}\right)} \leqq C \varepsilon^{\frac{1}{3}}
$$

Under an additional but natural non-degeneracy condition, satisfied by most potentials used in physical applications (recall the discussion in Section 1.2), we can improve estimate (39).

Proposition 7. If we assume that $W \in C^{2}$ and

$$
W_{t t}(0, \theta) \geqq c>0, \quad \theta \in[0, \ell),
$$

then

$$
\eta_{\varepsilon}(\boldsymbol{y})=\varepsilon^{\frac{1}{3}} \beta_{\varepsilon}(\theta) V\left(\beta_{\varepsilon}(\theta) \frac{t}{\varepsilon^{\frac{2}{3}}}\right)\left[1+\mathscr{O}\left(\varepsilon^{\frac{2}{3}}\right)\left(\frac{t}{\varepsilon^{\frac{2}{3}}}\right)^{\frac{5}{2}}\right],
$$

uniformly in $\left\{0 \leqq t \leqq d, \theta \in\left[0, \ell_{\varepsilon}\right)\right\}$, as $\varepsilon \rightarrow 0$, where $d>0$ is some small constant. 
Proof. Once again, we will work with the equivalent problem in stretched variables. Our aim is to estimate $\varphi_{*}=u_{\varepsilon}-u_{\text {ap }}$ using Equation (129), as we did for estimate (128). By virtue of (65), (73), (74), (83), and (101), for small $\varepsilon$, we get that the remainder in (130) satisfies

$$
|E| \leqq C \varepsilon x^{2} \mathrm{Ai} \quad \text { if } x \in\left[1,2 \delta \varepsilon^{-\frac{2}{3}}\right) .
$$

In view of (32), (107), and (177), it is easy to see that, decreasing $\delta$ if necessary, we have

$$
\varepsilon^{-\frac{2}{3}}\left(3 u_{\text {ap }}^{2}-a\left(\varepsilon^{\frac{2}{3}} y\right)\right) \geqq \beta^{2} x+c \varepsilon^{\frac{2}{3}} x^{2} \text { if } x \in\left[1,2 \delta \varepsilon^{-\frac{2}{3}}\right),
$$

provided $\varepsilon$ is sufficiently small. We point out that in the above relation the constant $c$ is independent of small $\delta, \varepsilon$. Let $\Psi>0$ be determined from

$$
-\Psi^{\prime \prime}+x \Psi=x^{2} \mathrm{Ai} ; \quad \Psi(1)=1, \quad \Psi(\infty)=0 .
$$

In order to proceed, we need some estimates for $\Psi$. A short calculation shows that

$$
\Psi=(\mathrm{Ai}) h \quad \text { with } h^{\prime}=\frac{\int_{x}^{\infty} t^{2}(\mathrm{Ai})^{2} \mathrm{~d} t}{(\mathrm{Ai})^{2}} .
$$

Note that, from (72), (74), we have

$$
\mathrm{Ai}^{\prime} \sim-x^{\frac{1}{2}} \mathrm{Ai} \text { as } x \rightarrow \infty .
$$

By the way, a neat way to show the above relation is to use L'hospital's rule to find that

$$
\lim _{x \rightarrow \infty} \frac{x^{-1}\left(\mathrm{Ai}^{\prime}\right)^{2}}{(\mathrm{Ai})^{2}}=1 .
$$

Now, referring to L'hospital's rule once more, we get

$$
h^{\prime} \sim \frac{1}{2} x^{\frac{3}{2}} \text { and } h \sim \frac{1}{5} x^{\frac{5}{2}} \quad \text { as } x \rightarrow \infty .
$$

Hence, we find that

$$
\Psi \sim \frac{1}{5} x^{\frac{5}{2}} \mathrm{Ai} \text { and } \Psi^{\prime} \sim-\frac{1}{5} x^{3} \mathrm{Ai} \quad \text { as } x \rightarrow \infty .
$$

Keeping in mind that $x=\beta\left(\varepsilon^{\frac{2}{3}} z\right) s$, via formulas (56)-(57), we get that

$$
\Delta_{y} \Psi=\beta^{2} \Psi^{\prime \prime}+\mathscr{O}\left(\varepsilon^{\frac{4}{3}}\right) x \Psi^{\prime}+\mathscr{O}\left(\varepsilon^{\frac{4}{3}}\right) x^{2} \Psi^{\prime \prime}+\mathscr{O}\left(\varepsilon^{\frac{2}{3}}\right) \Psi^{\prime},
$$

uniformly in $\left\{1 \leqq x \leqq 2 \delta \varepsilon^{-\frac{2}{3}}, \quad z \in\left[0, \ell_{\varepsilon} \varepsilon^{-\frac{2}{3}}\right)\right\}$, as $\varepsilon \rightarrow 0$ (note that here $\mathscr{O}(\cdot)$ is bounded uniformly in small $\delta$ ). Using (7), (110), (180)-(184), and further decreasing $\delta$, we readily find that

$$
\begin{aligned}
-\mathscr{L}_{\varepsilon}(\Psi) & \geqq \beta^{2} x^{2} \mathrm{Ai}+c \varepsilon^{\frac{2}{3}} x^{2} \Psi+\mathscr{O}\left(\varepsilon^{\frac{4}{3}}\right) x^{3} \Psi+\mathscr{O}\left(\varepsilon^{\frac{4}{3}}\right) x^{4} \mathrm{Ai}+\mathscr{O}\left(\varepsilon^{\frac{2}{3}}\right) x^{3} \mathrm{Ai} \\
& \geqq \frac{\beta^{2}}{2} x^{2} \mathrm{Ai}+c \varepsilon^{\frac{2}{3}} x^{2} \Psi
\end{aligned}
$$


if $x \in\left[1,2 \delta \varepsilon^{-\frac{2}{3}}\right)$, provided $\varepsilon$ is sufficiently small $(c, \mathscr{O}(\cdot)$ independent of small $\delta)$. It is nice to note that, in the above calculation, the cubic power in the second asymptotic relation of (183) was "the most appropriate" one in order to absorb the last term of (184) into the term $\beta^{2} x^{2} \mathrm{Ai}$, by decreasing $\delta$. Similarly, keeping in mind (72)-(74), we find that the function

$$
B(y) \equiv \operatorname{Bi}\left(x-2 \delta \varepsilon^{-\frac{2}{3}}\right), \quad y \in\left\{x \in\left[1,2 \delta \varepsilon^{-\frac{2}{3}}\right), z \in\left[0, \varepsilon^{-\frac{2}{3}} \ell_{\varepsilon}\right)\right\},
$$

satisfies

$$
\begin{aligned}
-\mathscr{L}_{\varepsilon}(B) & \geqq-\beta^{2} B^{\prime \prime}+\beta^{2} x B+c \varepsilon^{\frac{2}{3}} x^{2} B+\mathscr{O}\left(\varepsilon^{\frac{4}{3}}\right) x^{2}\left(x-2 \delta \varepsilon^{-\frac{2}{3}}\right) B+\mathscr{O}\left(\varepsilon^{\frac{2}{3}}\right) B^{\prime} \\
& \geqq 2 \delta \beta^{2} \varepsilon^{-\frac{2}{3}} B+c \varepsilon^{\frac{2}{3}} x^{2} B+\delta^{3} \mathscr{O}\left(\varepsilon^{-\frac{2}{3}}\right) B+\mathscr{O}\left(\varepsilon^{\frac{2}{3}}\right)\left(|x|^{\frac{1}{2}}+\delta^{\frac{1}{2}} \varepsilon^{-\frac{1}{3}}\right) B \\
& \geqq \delta \beta^{2} \varepsilon^{-\frac{2}{3}} B+c \varepsilon^{\frac{2}{3}} x^{2} B
\end{aligned}
$$

if $\varepsilon$ is sufficiently small, having further decreased $\delta$ if necessary. From now on we will fix $\delta$. In view of (131), (179), (185), and (187), given $M>1$, the function

$$
\Phi(\mathbf{y}) \equiv M \varepsilon\left\{\Psi(x)+\operatorname{Bi}\left(x-2 \delta \varepsilon^{-\frac{2}{3}}\right)\right\}
$$

satisfies

$$
\begin{aligned}
-\mathscr{L}_{\varepsilon}(\Phi)+N(\Phi)+E \geqq & M \frac{\beta^{2}}{2} \varepsilon x^{2} \mathrm{Ai}+c M \varepsilon^{\frac{5}{3}} x^{2} \Psi+M \delta \beta^{2} \varepsilon^{\frac{1}{3}} B+c M \varepsilon^{\frac{5}{3}} x^{2} B \\
& -C \varepsilon x^{2} \mathrm{Ai}-C M^{2} \varepsilon^{\frac{5}{3}} x^{5} V(\mathrm{Ai})^{2}-C M^{2} \varepsilon^{\frac{5}{3}} x^{\frac{5}{2}} V \mathrm{Ai} B \\
& -C M^{2} \varepsilon^{\frac{5}{3}} V B^{2}-C M^{3} \varepsilon^{\frac{7}{3}} x^{\frac{5}{2}} \mathrm{Ai}-C M^{3} \varepsilon^{\frac{7}{3}} B \\
\geqq & M \frac{\beta^{2}}{4} \varepsilon x^{2} \mathrm{Ai}+M \delta \beta^{2} \varepsilon^{\frac{1}{3}} B+c_{1} M \varepsilon^{\frac{5}{3}} x^{\frac{9}{2}} \mathrm{Ai} \\
& -C_{2} M^{2} \varepsilon^{\frac{5}{3}} x^{5} V(\mathrm{Ai})^{2},
\end{aligned}
$$

for some constants $c_{1}, C_{2}>0$ (independent of $\varepsilon$ ), if $\varepsilon<\varepsilon(M)$ is sufficiently small. Note that the seventh, eighth, and tenth term in the first inequality's righthand side were absorbed into the corresponding third term, whereas the ninth into the first. What we want to do next is to somehow "get rid" of the last term in the above relation, and end up with a positive righthand side. We will achieve this by absorbing that term into the one that proceeds it. By virtue of (73), (74), and (182), if $M$ is sufficiently large, there exists $x_{M}>0$ such that

$$
C_{2} M x^{\frac{1}{2}} V \mathrm{Ai}<c_{1} \text { if } x>x_{M} .
$$

(Note that $x_{M} \rightarrow \infty$ as $M \rightarrow \infty$.) Hence, it follows that

$$
-\mathscr{L}_{\varepsilon}(\Phi)+N(\Phi)+E \geqq M \delta \beta^{2} \varepsilon^{\frac{1}{3}} B+M \frac{\beta^{2}}{4} \varepsilon x^{2} \mathrm{Ai}>0
$$

in the strip-like domain described by

$$
\mathscr{S}_{\varepsilon}=\left\{x_{M} \leqq x \leqq 2 \delta \varepsilon^{-\frac{2}{3}}, \quad z \in\left[0, \ell_{\varepsilon} \varepsilon^{-\frac{2}{3}}\right)\right\} .
$$


Now, in view of (128), (183), and (188), we can fix a large $M>0$ such that

$$
\varphi_{*}<\Phi \quad \text { on }\left\{x=x_{M}\right\} ; \quad \varphi_{*}<\Phi \quad \text { on }\left\{x=2 \delta \varepsilon^{-\frac{2}{3}}\right\},
$$

if $\varepsilon$ is small. By (189), (190), and a standard maximum principle argument, making use of (106) and the property that

$$
\left|N(\Phi)-N\left(\varphi_{*}\right)\right| \leqq C \varepsilon^{\frac{2}{3}}\left|\Phi-\varphi_{*}\right| \quad \text { in } \mathscr{S}_{\varepsilon},
$$

we deduce that $\varphi_{*} \leqq \Phi$ in $\mathscr{S}_{\varepsilon}$, if $\varepsilon$ is small. Similarly we can show that $\varphi_{*} \leqq-\Phi$ in $\mathscr{S}_{\varepsilon}$, if $\varepsilon$ is small. The desired assertion of the proposition (for the equivalent stretched problem) now follows via (39), (73), (183), and noting that

$$
\mathrm{Bi}\left(x-2 \delta \varepsilon^{-\frac{2}{3}}\right) \leqq \mathrm{Bi}\left(-\delta \varepsilon^{-\frac{2}{3}}\right) \stackrel{(74)}{\leqq} 2 \mathrm{Ai}\left(\delta \varepsilon^{-\frac{2}{3}}\right) \leqq 2 \mathrm{Ai}(x)
$$

if $x \in\left[x_{M}, \delta \varepsilon^{-\frac{2}{3}}\right)$ with $\varepsilon$ sufficiently small.

The proof of the proposition is complete.

Remark 27. Note that the first term in the righthand side of (178) dominates for $0 \leqq t \ll \varepsilon^{\frac{2}{5}}$.

Assuming additionally that $W$ is radial and convex (outside of $\mathscr{D}_{\varepsilon}$ ), we can derive an explicit global upper bound on the minimizer.

Proposition 8. Assume that the potential trap $W$ is radially symmetric with

$$
W_{r r}\left(R_{\varepsilon}\right)>0, \text { and } W_{r r}(r) \geqq 0 \quad \text { if } r>R_{\varepsilon},
$$

where $R_{\varepsilon}$ denotes the radius of $\mathscr{D}_{\varepsilon}$. Then, we have

$$
\eta_{\varepsilon}(s) \leqq \frac{A i\left(\beta_{\varepsilon} \frac{s-R_{\varepsilon}}{\varepsilon^{\frac{2}{3}}}\right)}{A i\left(\beta_{\varepsilon} \frac{r-R_{\varepsilon}}{\varepsilon^{\frac{2}{3}}}\right)} \eta_{\varepsilon}(r) \quad \forall s \geqq r \geqq R_{\varepsilon} .
$$

In particular, it holds that

$$
\eta_{\varepsilon}(s) \leqq \varepsilon^{\frac{1}{3}}\left(\beta_{\varepsilon}+o(1)\right) A i\left(\beta_{\varepsilon} \frac{s-R_{\varepsilon}}{\varepsilon^{\frac{2}{3}}}\right) \text { if } s-R_{\varepsilon} \gg \varepsilon^{\frac{2}{3}},
$$

as $\varepsilon \rightarrow 0$.

Proof. Since

$$
-a_{\varepsilon}(r)=W(r)-W\left(R_{\varepsilon}\right) \geqq W_{r}\left(R_{\varepsilon}\right)\left(r-R_{\varepsilon}\right), \quad r \geqq R_{\varepsilon},
$$

we see that the minimizer $\eta_{\varepsilon}$ is a positive lower-solution of the linear equation

$$
-\varepsilon^{2}\left(\eta_{r r}+\frac{1}{r} \eta_{r}\right)+\beta_{\varepsilon}^{3}\left(r-R_{\varepsilon}\right) \eta=0,
$$


if $r \geqq R_{\varepsilon}$ (recall that $\beta_{\varepsilon}=\left[W_{r}\left(R_{\varepsilon}\right)\right]^{\frac{1}{3}}>0$ ). On the other side, making use of (72) and the fact that $(\mathrm{Ai})^{\prime}<0$, we readily find that $\mathrm{Ai}\left(\beta_{\varepsilon} \frac{r-R_{\varepsilon}}{\varepsilon^{\frac{2}{3}}}\right)$ is a positive upper-solution of (193) if $r \geqq R_{\varepsilon}$. Hence, by the maximum principle, we deduce that relation (191) holds true. In turn, via (39), (73), (76), and Proposition 7, relation (191) implies the validity of relation (192).

The proof of the proposition is complete.

Remark 28. It seems plausible that the techniques of the very recent paper [60] can be extended to derive a WKB (Wentzel-Kramers-Brillouin) type estimate, in the region $(R, \infty)$ (with the obvious notation), for the ground state solution of (22) with $q=2$ and $N=1$.

\section{Refined Estimates for the Auxiliary Functions $\xi_{\varepsilon}, f_{\varepsilon}$ in the Case of Radial Symmetry}

In this section, restricting ourselves to radial potentials with $\mathscr{D}_{0}$ a ball, building on our previous results for the ground state $\eta_{\varepsilon}$, we will improve upon the estimates obtained recently in [10] for the auxiliary function $f_{\varepsilon}$ in (31). As we have already discussed in Section 1.3, the latter estimates were essential for the analysis of [10] regarding the functional $E_{\varepsilon}$, defined in (15). We believe that the improved estimates herein may provide important intuition for the treatment of the general case, which may ultimately lead to the resolution of the open problem raised in [10] (recall the discussion in Section 1.3).

In the general case, for potentials as described in Section 1.1, we define $\xi_{\varepsilon}$ to be the solution of

$$
\operatorname{div}\left(\frac{1}{\eta_{\varepsilon}^{2}} \nabla \xi\right)=-2, \quad \mathbf{y} \in \mathbb{R}^{2}, \quad \xi_{\varepsilon}(\mathbf{y}) \rightarrow 0,|\mathbf{y}| \rightarrow \infty
$$

so that $\nabla^{\perp} \xi_{\varepsilon}=x^{\perp} \eta_{\varepsilon}^{2}$. An integration by parts in (19) yields

$$
F_{\varepsilon}(w)=\int_{\mathbb{R}^{2}}\left\{\frac{\eta_{\varepsilon}^{2}}{2}\left(|\nabla w|^{2}-\frac{4 \Omega \xi_{\varepsilon}}{\eta_{\varepsilon}^{2}} J w\right)+\frac{\eta_{\varepsilon}^{4}}{4 \varepsilon^{2}}\left(|w|^{2}-1\right)^{2}\right\} \mathrm{d} \mathbf{y}
$$

where $J w=\frac{1}{2} \nabla \times(i w, \nabla w)=\left(i w_{\mathbf{y}_{1}}, w_{\mathbf{y}_{2}}\right)$ is the Jacobian.

We recall that the function $f_{\varepsilon}:=\xi_{\varepsilon} / \eta_{\varepsilon}^{2}$, appearing in the functional $F_{\varepsilon}$, is important since it is well known that vortices in the interior of $\mathscr{D}_{0}$ first appear near where this function attains a local maximum $[8,9,125,126]$; its importance is also clear from (195), since it controls the relative strength of the positive and negative contributions to $F_{\varepsilon}$.

In the case where the potential $W$ is radially symmetric, one can solve problem (194) explicitly to find that the functions $\xi_{\varepsilon}, f_{\varepsilon}$ are given by relation (31). In particular, if the domain $\mathscr{D}_{0}$ is a ball, it has been shown in [10] that, for small $\varepsilon$, we 
have

$$
f_{\varepsilon}(|\mathbf{y}|) \leqq\left\{\begin{array}{ll}
C \operatorname{dist}\left(\mathbf{y}, \partial \mathscr{D}_{0}\right)+C \varepsilon^{\frac{2}{3}} & \text { if } \mathbf{y} \in \mathscr{D}_{0}, \\
C \varepsilon^{\frac{2}{3}} & \text { if not, }
\end{array} \quad \text { and }\left\|f_{\varepsilon}-f_{0}\right\|_{L^{\infty}(\mathbb{R})} \leqq C \varepsilon^{\frac{1}{3}}\right.
$$

where $f_{0}$ is the function in (200) below, which solves the "limiting" problem corresponding to (194):

$$
\operatorname{div}\left(\frac{1}{A} \nabla \xi\right)=-2 \text { in } \mathscr{D}_{0}, \quad \xi_{\varepsilon}=0 \quad \text { on } \partial \mathscr{D}_{0},
$$

with $A$ as in (9). The existence and properties of a positive solution $\xi_{0}$ of (197) have been established in [12]. The following proposition refines and improves relation (196).

Proposition 9. If the potential $W$ is radially symmetric with $\mathscr{D}_{0}=\left\{\boldsymbol{y} \in \mathbb{R}^{2}: r=\right.$ $|\boldsymbol{y}|<R\}$, then the function $f_{\varepsilon}$, defined in (31), satisfies

$$
f_{\varepsilon}(r)=R_{\varepsilon} \beta_{\varepsilon}^{-1} \varepsilon^{\frac{2}{3}} V^{-2}\left(\beta_{\varepsilon} \frac{r-R_{\varepsilon}}{\varepsilon^{\frac{2}{3}}}\right) \int_{\beta_{\varepsilon} \frac{r-R_{\varepsilon}}{\varepsilon^{\frac{2}{3}}}}^{\infty} V^{2}(\sigma) \mathrm{d} \sigma+o\left(\varepsilon^{\frac{2}{3}}\right)
$$

uniformly in $\left[R-o\left(\varepsilon^{\frac{1}{3}}\right), \infty\right)$, as $\varepsilon \rightarrow 0$, where $R_{\varepsilon}$ denotes the radius of the ball $\mathscr{D}_{\varepsilon}$, satisfying (201) below, and $\beta_{\varepsilon}=\left[W^{\prime}\left(R_{\varepsilon}\right)\right]^{\frac{1}{3}}[$ recall (36) $]$.

Moreover, if $\varepsilon$ is small, it holds that

$$
\left\|f_{\varepsilon}-f_{0}\right\|_{L^{\infty}(\mathbb{R})} \leqq C \varepsilon^{\frac{1}{2}}
$$

where

$$
f_{0}(r)= \begin{cases}\frac{1}{A(r)} \int_{r}^{R} s A(s) \mathrm{d} s, & 0 \leqq r<R \\ 0, & r \geqq R\end{cases}
$$

(In view of (6), and (7), an application of L'hospital's rule shows that $f_{0}^{\prime}\left(R^{-}\right)=$ $\left.-\frac{R}{2}\right)$.

Proof. First of all note that, thanks to (45), we have

$$
R_{\varepsilon}=R+\mathscr{O}\left(\varepsilon^{\frac{4}{3}}\right) \text { as } \varepsilon \rightarrow 0 .
$$

By virtue of (4), (6), (7), and (45), there exists a constant $c>0$ such that, given $K \gg 1$, we have

$$
\eta_{\varepsilon}^{2}+W(r)-\lambda_{\varepsilon} \geqq W(r)-\lambda_{\varepsilon} \geqq c(r-R)^{2}+c K \varepsilon^{\frac{2}{3}}, \quad r \geqq R+K \varepsilon^{\frac{2}{3}},
$$

provided that $\varepsilon$ is sufficiently small. (We note that $K$ is considered fixed in the corresponding relation in [10]). Then, by a standard barrier argument in equation (8), we deduce that

$$
\eta_{\varepsilon}(s) \leqq \eta_{\varepsilon}(r) \mathrm{e}^{-K^{\frac{1}{3}} \varepsilon^{-\frac{2}{3}}\left(s^{2}-r^{2}\right)}, \quad s \geqq r \geqq R+K \varepsilon^{\frac{2}{3}},
$$


if $K$ is sufficiently large and $\varepsilon$ sufficiently small. As a result, we get

$$
f_{\varepsilon}(r) \leqq \int_{r}^{\infty} s e^{-2 K^{\frac{1}{3}} \varepsilon^{-\frac{2}{3}}\left(s^{2}-r^{2}\right)} \mathrm{d} s=\frac{1}{4} K^{-\frac{1}{3}} \varepsilon^{\frac{2}{3}}, \quad r \geqq R+K \varepsilon^{\frac{2}{3}},
$$

if $\varepsilon$ is small.

If $r \in\left[R-d^{\prime}, R+K \varepsilon^{\frac{2}{3}}\right]$, in view of Corollary 2 and (202), for small $\varepsilon$, we have

$$
\begin{aligned}
f_{\varepsilon}(r) & =\frac{1}{\eta_{\varepsilon}^{2}(r)} \int_{r}^{R+K \varepsilon^{\frac{2}{3}}} s \eta_{\varepsilon}^{2}(s) \mathrm{d} s+\frac{\eta_{\varepsilon}^{2}\left(R+K \varepsilon^{\frac{2}{3}}\right)}{\eta_{\varepsilon}^{2}(r)} f_{\varepsilon}\left(R+K \varepsilon^{\frac{2}{3}}\right) \\
& =\frac{1}{\eta_{\varepsilon}^{2}(r)} \int_{r}^{R+K \varepsilon^{\frac{2}{3}}} s \eta_{\varepsilon}^{2}(s) \mathrm{d} s+\mathscr{O}\left(K^{-\frac{1}{3}} \varepsilon^{\frac{2}{3}}\right)
\end{aligned}
$$

uniformly as $\varepsilon \rightarrow 0$. If $r \in\left[R, R+K \varepsilon^{\frac{2}{3}}\right]$, from (39), (68), and (201), it follows readily that

$$
\begin{aligned}
& \frac{1}{\eta_{\varepsilon}^{2}(r)} \int_{r}^{R+K \varepsilon^{\frac{2}{3}}} s \eta_{\varepsilon}^{2}(s) \mathrm{d} s=R_{\varepsilon} \beta_{\varepsilon}^{-1} \varepsilon^{\frac{2}{3}} V^{-2}\left(\beta_{\varepsilon} \frac{r-R_{\varepsilon}}{\varepsilon^{\frac{2}{3}}}\right) \int_{\beta_{\varepsilon} \frac{r-R_{\varepsilon}}{\varepsilon^{\frac{2}{3}}}}^{\infty} V^{2}(\sigma) \mathrm{d} \sigma \\
& +\mathscr{O}\left(\varepsilon^{\frac{2}{3}} V^{-2}(K) \int_{K}^{\infty} V^{2}(\sigma) \mathrm{d} \sigma\right)+\mathscr{O}_{K}\left(\varepsilon^{\frac{4}{3}}\right),
\end{aligned}
$$

uniformly, as $\varepsilon \rightarrow 0$ (the constant $\mathscr{O}_{K}(1)$ may diverge as $K \rightarrow \infty$ ). The second term in the righthand side of the above relation can be estimated as before, by noting that, thanks to (65), we find that

$$
V(\sigma) \leqq V(K) \mathrm{e}^{-K^{\frac{1}{3}}(\sigma-K)}, \quad \sigma \geqq K \text { if } K \text { is large. }
$$

Thus, by (203), (204), we infer that relation (198) holds true in $\left[R, R+K \varepsilon^{\frac{2}{3}}\right]$. In fact, by (202) and the above relation, we deduce that (198) holds true in $\left[R+K \varepsilon^{\frac{2}{3}}, \infty\right)$ as well. If $r \in[R-d, R]$, similarly as before, but this time using (38) instead of (39), we arrive at

$$
\begin{aligned}
f_{\varepsilon}(r)= & R_{\varepsilon} \beta_{\varepsilon}^{-1} \varepsilon^{\frac{2}{3}} V^{-2}\left(\beta_{\varepsilon} \frac{r-R_{\varepsilon}}{\varepsilon^{\frac{2}{3}}}\right) \int_{\beta_{\varepsilon} \frac{r-R_{\varepsilon}}{\varepsilon^{\frac{2}{3}}}}^{\infty} V^{2}(\sigma) \mathrm{d} \sigma \\
& +\mathscr{O}\left(\left|r-R_{\varepsilon}\right|^{2}+\varepsilon\left|r-R_{\varepsilon}\right|^{\frac{1}{2}}+\varepsilon^{\frac{1}{3}}\left|r-R_{\varepsilon}\right|^{\frac{3}{2}}\right)+o\left(\varepsilon^{\frac{2}{3}}\right),
\end{aligned}
$$

uniformly, as $\varepsilon \rightarrow 0$. The above relation implies at once the validity of (198) in $\left[R-o\left(\varepsilon^{\frac{1}{3}}\right), R\right]$ as $\varepsilon \rightarrow 0$. Consequently, we have established the validity of (198).

Next, we will show the validity of estimate (199). If $r \in\left[R-\varepsilon^{\frac{2 \alpha}{3}}, R\right]$, with $\frac{1}{2}<\alpha \leqq 1$, recalling (7), we have $c(R-r) \leqq A(r) \leqq C(R-r)$. So, as in [10], we obtain that

$$
f_{0}(r) \leqq \frac{C}{R-r} \int_{r}^{R} s(R-s) \mathrm{d} s \leqq C(R-r) \leqq C \varepsilon^{\frac{2 \alpha}{3}} .
$$


Furthermore, thanks to (68), (198), if $\varepsilon$ is small, we find that

$$
f_{\varepsilon}(r) \leqq C \varepsilon^{\frac{2 \alpha}{3}}, \quad r \in\left[R-\varepsilon^{\frac{2 \alpha}{3}}, R\right]
$$

If $r \in\left[0, R-\varepsilon^{\frac{2 \alpha}{3}}\right]$, following [10], we write

$$
\begin{aligned}
f_{\varepsilon}(r)-f_{0}(r)= & \left\{\frac{1}{\eta_{\varepsilon}^{2}(r)} \int_{r}^{R-\varepsilon^{\frac{2 \alpha}{3}}} s \eta_{\varepsilon}^{2}(s) \mathrm{d} s-\frac{1}{A(r)} \int_{r}^{R-\varepsilon^{\frac{2 \alpha}{3}}} s A(s) \mathrm{d} s\right\} \\
& +\frac{\eta_{\varepsilon}^{2}\left(R-\varepsilon^{\frac{2 \alpha}{3}}\right)}{\eta_{\varepsilon}^{2}(r)} f_{\varepsilon}\left(R-\varepsilon^{\frac{2 \alpha}{3}}\right)-\frac{A\left(R-\varepsilon^{\frac{2 \alpha}{3}}\right)}{A(r)} f_{0}\left(R-\varepsilon^{\frac{2 \alpha}{3}}\right) \\
= & \mathrm{I}+\mathrm{II}-\mathrm{III} .
\end{aligned}
$$

Using (6), (7), (40), Corollary 2, and our earlier estimates on $f_{\varepsilon}, f_{0}$ for $r \geqq R-\varepsilon^{\frac{2 \alpha}{3}}$, we see that

$$
|\mathrm{II}| \leqq C f_{\varepsilon}\left(R-\varepsilon^{\frac{2 \alpha}{3}}\right) \leqq C \varepsilon^{\frac{2 \alpha}{3}} \text { and }|\mathrm{III}| \leqq C f_{0}\left(R-\varepsilon^{\frac{2 \alpha}{3}}\right) \leqq C \varepsilon^{\frac{2 \alpha}{3}}
$$

We further decompose the remaining term as

$\mathrm{I}=\left(\frac{1}{\eta_{\varepsilon}^{2}(r)}-\frac{1}{A(r)}\right) \int_{r}^{R-\varepsilon^{\frac{2 \alpha}{3}}} s \eta_{\varepsilon}^{2}(s) \mathrm{d} s+\frac{1}{A(r)} \int_{r}^{R-\varepsilon^{\frac{2 \alpha}{3}}} s\left(\eta_{\varepsilon}^{2}(s)-A(s)\right) \mathrm{d} s$

Using Corollary 6 , if $\frac{1}{2}<\alpha<1$, for small $\varepsilon$, it follows that

$$
|\mathrm{I}| \leqq C \varepsilon^{2-2 \alpha} \int_{r}^{R-\varepsilon^{\frac{2 \alpha}{3}}} s \frac{\eta_{\varepsilon}^{2}(s)}{\eta_{\varepsilon}^{2}(r)} \mathrm{d} s+C \varepsilon^{2-2 \alpha} \int_{r}^{R-\varepsilon^{\frac{2 \alpha}{3}}} s \frac{A(s)}{A(r)} \mathrm{d} s .
$$

Due to Corollary 2, we have $\frac{\eta_{\varepsilon}^{2}(s)}{\eta_{\varepsilon}^{2}(r)} \leqq 1$ if $R-d^{\prime} \leqq r \leqq s \leqq R-\varepsilon^{\frac{2 \alpha}{3}}$. If $0 \leqq r \leqq R-d^{\prime}$, then $\eta_{\varepsilon}^{2}(r) \geqq c$, and so $\frac{\eta_{\varepsilon}^{2}(s)}{\eta_{\varepsilon}^{2}(r)} \leqq C$. Thus, the first integral in the above relation is bounded by $C \varepsilon^{2-2 \alpha}$. The second integral is estimated similarly, using (7) instead of Corollary 2. Therefore, relation (208) implies that

$$
\left|f_{\varepsilon}(r)-f_{0}(r)\right| \leqq C \varepsilon^{2-2 \alpha}, \quad r \in\left[0, R-\varepsilon^{\frac{2 \alpha}{3}}\right]
$$

provided that $\varepsilon$ is sufficiently small. The validity of estimate (199) follows at once by combining (206), (207), the above relation, and choosing $\alpha=\frac{3}{4}$.

The proof of the proposition is complete. 


\section{Open Problems and Future Directions}

What follows is a list of questions which are currently unresolved. These are presented as an illustration of where our interests lie. No attempt is being made to be precise in their formulation.

A question that comes naturally to mind is to examine whether the estimates of Theorem 1 , for the minimizer $\eta_{\varepsilon}$ of $G_{\varepsilon}$ in $\mathscr{H}$, can be used to answer the interesting open problem posed recently in [10]. As we have already mentioned in Section 1.3, the latter is to see to what extend the analysis of [10], for the functional $E_{\varepsilon}$ in (15), continues to hold if one drops the assumption of radial symmetry on the potential $W$. Hopefully, our estimates for $\eta_{\varepsilon}$ can be used in estimating the corresponding auxiliary functions $\xi_{\varepsilon}, f_{\varepsilon}$, arising in the functional $E_{\varepsilon}$ as in (195), which for the radial case were given by (31). It seems that the elliptic problem (194), which determines $\xi_{\varepsilon}$, seems to be a singular perturbation problem of its own independent interest.

In the special case of the model harmonic potential, an approximate solution for (22)_, "close" to $\sqrt{(\lambda-W)^{+}}$, of arbitrary order accuracy was constructed in [104] (keep in mind Remark 21). We feel that it would be very interesting if one can do the same thing for the case of general potential. A major difficulty (or problem) is that each term of the inner expansion diverges polynomially in a complicated manner as the distance from $\Gamma$ increases (recall Remark 14), see also the appendix in [55]. The construction of arbitrary order approximations is especially important in the treatment of singularly perturbed elliptic problems involving resonance, where the order of accuracy of the approximation is dictated by the space dimension, see for instance [160]. Problems of these type which feature the presence of a corner layer (similar to the problem at hand) have been studied recently in [135] (in two dimensions), see also Remark [114] herein. It should be noticed that in Allen-Cahn or (focusing) Schrödinger type equations, where its possible to construct arbitrary order approximations (see [160] and the references therein), the phenomenon is exponentially localized, i.e, the corresponding terms approach certain constants exponentially fast.

Relation (44) implies that the spectrum, in $L^{2}\left(\mathbb{R}^{2}\right)$, of the operator $\mathbf{L}_{\varepsilon}$, defined in (43), is bounded above by $-c \varepsilon^{\frac{2}{3}}$, for some constant $c>0$, as $\varepsilon \rightarrow 0$. We expect that, making further use of the estimates of Theorem 1, one can rigorously "link" the spectrum of $\mathbf{L}_{\varepsilon}$ to that of the one-dimensional "limit" operator $\mathscr{M}$, defined in (70), as $\varepsilon \rightarrow 0$ [see also relations (209)-(210) below], and thus provide a valid asymptotic approximation for the eigenvalues of $\mathbf{L}_{\varepsilon}$. In particular, the difference between the first two eigenvalues, called the fundamental gap, is of importance since it determines the rate at which positive solutions of the nonlinear heat equation, corresponding to (22)_, approach the first eigenspace of $\mathbf{L}_{\varepsilon}$ (see [28,118], and especially [44]). In the case where $W$ is the harmonic potential, a rigorous connection between the spectrum of $\mathbf{L}_{\varepsilon}$ and that of $\mathscr{M}$ [see the discussion following relation (A.231) below], as $\varepsilon \rightarrow 0$, has been made recently in [104] (see also [134] for a related radially symmetric problem). A possible approach for the general case, where the potential is as in the present paper, could be by mixing techniques found in the aforementioned references with those developed in [17,59] for the study 
of the spectrum of multi-dimensional Allen-Cahn and related phase-field operators for generic interfaces. One could even carry out an analogous program for the spectrum of the linearization of the defocusing nonlinear Schrödinger equation (21)_ at the corresponding ground state, recall (47). The latter problem is often referred to as the Bogoliubov-de Gennes problem in the context of Bose-Einstein condensates, see $[103,137]$ for recent studies specializing on the model harmonic potential. In the latter references, for reducing the complexity of the problem, the authors linearized at $\eta_{0}$ [recall (24)] instead of the ground state $\eta_{\varepsilon}$. In this case, the linear operator defined by the left hand side of the first equation in (47), with $\eta_{0}$ in place of $\eta_{\varepsilon}$, has also been studied in [101], in relation with [191].

Excited states are solutions of (22) _ with zero set inside the domain $\mathscr{D}_{\lambda} \equiv\{\mathbf{y}$ : $W(\mathbf{y})<\lambda\}$. In the Thomas-Fermi limit, $\varepsilon \rightarrow 0$, the Bose-Einstein condensate is a nearly compact cloud, which may contain localized dips of the atomic density. The nearly compact cloud is modeled by the ground state of the defocusing nonlinear Schrödinger equation (21)_, whereas the localized dips are modeled by the excited states. In the one-dimensional case, with $W$ the harmonic potential, excited states of (22)_ which are approximated, as $\varepsilon \rightarrow 0$, by a product of the ground state and $m$ dark solitons [localized waves of the defocusing NLS equation with nonzero boundary conditions at respective infinities, which after a re-scaling solve the onedimensional (49)] were constructed in [174] by a finite-dimensional LyapunovSchmidt reduction (for the latter see for instance the book [20]). Loosely speaking, these solutions have a corner layer at the points corresponding to $\partial \mathscr{D}_{\lambda}$, and $m$ (clustering) transition layers in $(-C|\ln \varepsilon| \varepsilon, C|\ln \varepsilon| \varepsilon$ ), as $\varepsilon \rightarrow 0$ (see also [62]). Studies in the case of radial symmetry have been conducted in [119]. We believe that, at least in two space dimensions, analogous excited states can still be constructed without any symmetry assumptions on the potential, by employing the estimates of Theorem 1 [in particular (44)] and the infinite dimensional Lyapunov-Schmidt reduction of [79] (see also [201]). In this context, the dimension $N=2$ plays an important role for the solvability of a Toda system, periodic orbits of which determine, up to principal order, the location of $m$ closed curves in $\mathscr{D}_{\lambda}$ where the excited state changes sign. These curves should collapse, as $\varepsilon \rightarrow 0$, to a closed curve in $\mathscr{D}_{\lambda}$ that may be determined by the arguments in $[86,151,166]$ (if $N=1$, the interfaces collapse at critical points of $W$ ). We expect that, in the case at hand, the reduction procedure is more delicate than [79] because the corresponding linear operator $\mathbf{L}_{\varepsilon}$ has small eigenvalues (see also [71] for a related finite-dimensional reduction). If $N=1$ or $W$ is radial, one could also try to construct "high energy" excited states of (22) $\varepsilon \rightarrow 0$, in the spirit of $[88,89]$ and the references therein. We remark that the result of [89] relied on ODE techniques, but it is expectable that a similar result could be proven for higher-dimensional problems. On the other hand, in the one-dimensional case, solutions of (22) _ bifurcating from the trivial branch have been studied in [146,187], and [196] (see also [139] and the references therein). Let us make a formal connection between these two different types of solutions (layered and small amplitude respectively). Consider the one-dimensional case with potential $W$ having a global minimum which is attained at a unique point, say at $\mathbf{y}=0$, and 
satisfying

$$
W(\mathbf{y})=W(0)+c|\mathbf{y}|^{\alpha}+o\left(|\mathbf{y}|^{\alpha}\right) \text { as } \mathbf{y} \rightarrow 0,
$$

for some constants $\alpha, c>0$. Arguing as in [134, Prop. 3.25], it is not hard to establish that, given $m \in \mathbb{N}$, the first $m$ eigenvalues of the linear operator

$$
-\varepsilon^{2} \partial_{\mathbf{y y}}+(W(\mathbf{y})-\lambda) I
$$

which corresponds to the linearization of (22) about the trivial solution, are of the form

$$
W(0)-\lambda+\mu_{i} \varepsilon^{\frac{2 \alpha}{\alpha+1}}+o\left(\varepsilon^{\frac{2 \alpha}{\alpha+1}}\right) \quad \text { as } \varepsilon \rightarrow 0, i=1, \ldots, m
$$

where $\left\{\mu_{i}\right\}$ are the eigenvalues of the "limit" operator

$$
-\partial_{\mathbf{y y}}+c|\mathbf{y}|^{\alpha} I
$$

(these exist by [121, Thm. 10.7], and $\mu_{i} \rightarrow \infty$ as $i \rightarrow \infty$ ). In passing, we not that formulas (210) improve the corresponding lower bounds found in Theorem 1.4 of [101]. Hence, we see that the number of negative eigenvalues (counting multiplicities), namely the Morse index of the trivial solution of (22)_ (see [134] for the precise definition), diverges as $\varepsilon \rightarrow 0$ (recall that $\lambda>W(0)$ ). From a variant of Weyl's asymptotic formula, see for example [32, pg. 521], it turns out that one has

$$
\mu_{i} \sim c i^{\frac{2 \alpha}{\alpha+2}} \text { as } i \rightarrow \infty
$$

for some constant $c>0$. We expect that, by refining the above argument, one can prove that the Morse index of the trivial solution is of order greater than or equal to $1 / \varepsilon$, as $\varepsilon \rightarrow 0$ (keep in mind that Landau's symbol in (210) may depend on $m \gg 1$ ). On the other side, it seems plausible that the operator in (209) does not have any negative eigenvalues if $\varepsilon$ is sufficiently large (by Poincaré's inequality, this is certainly true when considered in a fixed interval with Dirichlet boundary conditions). Consequently, since the eigenvalues are smooth functions of $\varepsilon$ (by virtue of their simplicity [61, Th. 3.1, p. 482], see also [160]), we expect that there exists a sequence $\left\{\varepsilon_{i}\right\}$ with $\varepsilon_{1}>\varepsilon_{2}>\cdots>\varepsilon_{i} \rightarrow 0$ as $i \rightarrow \infty$ such that, for each $\varepsilon=\varepsilon_{i}$, zero is an eigenvalue of the linearized operator described in (209). This suggests that the aforementioned local bifurcation of solutions of (22) that, using global bifurcation techniques [178] (see also [2]), one can show that these solution branches reach, as $\varepsilon \rightarrow 0$, the layered solutions of (22) - that we discussed previously. (We point out that solutions belonging to the $i$-th branch have exactly $i-1$ zeros). We note that analogous eigenvalues of the form (210), with the obvious modifications, also exist in the multi-dimensional case, and existence of many solutions for the nonlinear problem may follow by adapting Theorem 10.22 in [21]. Moreover, in the "flat" case (motivated from a definition in [47], see also [147]), where the potential $W$ attains its minimum value over a domain $\Omega_{0}$, we 
expect that the multi-dimensional operator, corresponding to (209), has eigenvalues of the form

$$
W(0)-\lambda+\mu_{i}\left(\Omega_{0}\right) \varepsilon^{2}+o\left(\varepsilon^{2}\right) \text { as } \varepsilon \rightarrow 0,
$$

where $\left\{\mu_{i}\left(\Omega_{0}\right)\right\}$ are the Dirichlet eigenvalues of $-\Delta$ in $\Omega_{0}$. We believe that the eigenfunctions associated to the above eigenvalues and the corresponding eigenfunctions of the Laplacian share the topology of their level sets, as in [90]. In any case, motivated from results in [11], we believe that the existence and multiplicity of solutions to $(22)_{-}$, is strongly associated to the number of negative eigenvalues (counting multiplicities) of the corresponding linearized operator about the trivial solution. In the radially symmetric case, the topological approaches of $[111,187]$, for constructing nodal standing wave solutions of the focusing NLS, should also be applicable to the defocusing case with a trapping potential, see also a related remark in [162].

It would also be interesting if one can find an asymptotic expansion, as $\varepsilon \rightarrow 0$, of the energy $E_{1}$ of the first excited state (with least energy), as we did for the energy $E_{g}$ of the ground state in Theorem 1. The difference $E_{1}-E_{g}$ is of importance since it represents the "excitation energy" required to reach the first excited state from the ground state; it thus determines in some sense the stability of the ground state. (In the case of a convex bounded domain with Dirichlet boundary conditions, with the obvious modifications, this would provide evidence on the validity of a "nonlinear fundamental gap conjecture", see [24,31].)

If in addition the potential $W$ is assumed to be even with respect to the coordinate axis, we have observed that one can construct a sign changing solution of (22) _, whose nodal set is the union of the coordinate axis, using the following strategy: firstly, by minimizing the functional $\mathscr{G}_{-}$, described in (23), over $\eta \in W_{0}^{1,2}\left(\mathbb{R}_{+}^{2}\right)$ such that $W \eta^{2} \in L^{1}\left(\mathbb{R}_{+}^{2}\right)$, where $\mathbb{R}_{+}^{2} \equiv\left\{\mathbf{y}=\left(\mathbf{y}_{1}, \mathbf{y}_{2}\right): \mathbf{y}_{1}>0, \mathbf{y}_{2}>0\right\}$, for small $\varepsilon>0$, we obtain a positive solution in $\mathbb{R}_{+}^{2}$ of the equation in (22)_ which is zero on the coordinate axis and approaches zero as $|\mathbf{y}| \rightarrow \infty$ (we can see that the minimizer is nontrivial, if $\varepsilon$ is small, by adapting Example 5.11 in [21] or Lemma 2.1 in [82]). A solution $\mathbf{u}_{2}$ defined in the entire space is then obtained using odd reflections through the lines $\mathbf{y}_{1}=0$ and $\mathbf{y}_{2}=0$. The function $\mathbf{u}_{2}$ is a solution of (22) 0-level set is the union of the two axis. Our construction parallels that of the well known saddle solution of the Allen-Cahn equation (49), see [74]. The problem of existence and qualitative properties of saddle type solutions for the Allen-Cahn equation (not necessarily in two dimensions) has received a considerable amount of attention in recent years, see [50-52,144]. We wonder if an analogous study can be conducted for the saddle type solutions of (22) _ that we just described. Can one rigorously verify the formal prediction that

$$
\mathbf{u}_{2} \rightarrow \operatorname{sign}\left\{\mathbf{y}_{1} \mathbf{y}_{2}\right\} \sqrt{(\lambda-W)^{+}},
$$

say in $L^{2}\left(\mathbb{R}^{2}\right)$, as $\varepsilon \rightarrow 0$ ? The finer structure at the junction points on the axis, where $W=\lambda$, may be demonstrated by a solution $v$ of the following elliptic problem:

$$
\left\{\begin{array}{l}
v_{x x}+v_{z z}-\left(x+v^{2}\right) v=0, \quad x \in \mathbb{R}, \quad z>0, \\
v-\sqrt{-x} \rightarrow 0 \text { as } x \rightarrow-\infty ; \quad v \rightarrow 0 \text { as } x \rightarrow \infty, \\
v=0 \text { if } z=0 ; \quad v-V(x) \rightarrow 0 \text { as } z \rightarrow \infty,
\end{array}\right.
$$


where $V$ denotes the Hastings-McLeod solution as usual, which seems to be of independent interest. The above can be generalized to the case of arbitrary even space dimensions. Let us also note that, if the potential trap $W$ is radial and twodimensional, our construction can easily be generalized to obtain solutions $\mathbf{u}_{k}$ of (22)_ with $N=2$, for small $\varepsilon>0$, whose zero level set has the symmetry of a regular $2 k$-polygon and consists of $k$ straight lines passing through the origin (see [15] for the corresponding solutions of (49)).

In the case where the potential $W$ is, say, two-dimensional and symmetric with respect to the coordinate axis (as in the above paragraph) but the equation of (22) is posed in $\mathbb{R}^{3}$, motivated from a definition in [102], we can also consider "tick" saddle solutions. As before, minimizing the functional $\mathscr{G}_{-}$over $\eta \in W_{0}^{1,2}(\Omega)$ such that $W \eta^{2} \in L^{1}(\Omega)$, where $\left.\Omega \equiv\left\{\mathbf{y}_{1}>0, \mathbf{y}_{2}>0,-D<\mathbf{y}_{3}<D\right)\right\}$, $D>0$, for $\varepsilon<\varepsilon(D)$ sufficiently small, yields a positive solution of the equation which vanishes on $\partial \Omega=\left\{\mathbf{y}_{1}=0, \mathbf{y}_{2}=0, \mathbf{y}_{3}= \pm D\right\}$. By odd reflection with respect to $\left\{\mathbf{y}_{1}=0, \mathbf{y}_{2}=0, \mathbf{y}_{3} \in(-D, D)\right\}$, and then with respect to the planes $\mathbf{y}_{3}=(2 k+1) D, k \in \mathbb{Z}$, that solution can be extended to the whole of $\mathbb{R}^{3}$, yielding an entire solution of the equation in (22)_ which has a saddle structure on each plane $\mathbf{y}_{3}=$ constant and is periodic of period $4 D$ in the $\mathbf{y}_{3}$ variable.

Very recently, del Pino, Musso, and Pacard [82] studied entire solutions of the Allen-Cahn equation (49) which are defined in 3-dimensional Euclidean space and which are invariant under screw-motion. In particular, their nodal set is a helicoïd of $\mathbb{R}^{3}$. We believe that, for sufficiently small $\varepsilon>0$, similar solutions exist for the 3-dimensional defocusing Gross-Pitaevskii equation in (22) - with a 2-dimensional radial potential $W$ ( $\lambda, W$ satisfying our usual assumptions). What is the asymptotic behavior of these solutions as $\varepsilon \rightarrow 0$ ? Can some results of [82] be generalized in our context?

We believe that, if the potential $W$ is restricted to the radial class, the approach of the current paper can also be applied to the study of the $\varepsilon \rightarrow 0$ limiting behavior of vortex solutions of the NLS equation (21)_, see [142] or [185], namely solutions of the form

$$
\left\{\begin{array}{l}
u_{n}(\mathbf{y}, t)=U_{n}(r) \mathrm{e}^{i(n \theta-\lambda t / \varepsilon)}, \quad n= \pm 1, \pm 2, \ldots, \\
U_{n}(0)=0, \quad U_{n}(\infty)=0,
\end{array}\right.
$$

where $(r, \theta)$ denote the polar coordinates in $\mathbb{R}^{2}$. Hopefully, the obtained estimates could be used to prove the, indicated by numerical evidence [137] (for the case of the model harmonic potential), orbital stability of $u_{1}$ in the time evolution of the Gross-Pitaevskii equation, and thus answering the question raised in the end of the recent paper [175].

Non-degeneracy conditions of the form (7) are common in the study of transition layered solutions of elliptic equations with bistable nonlinearity, see [96,201]. In that context, the surface $\partial \mathscr{D}_{0}$ represents the interface of the layer. It turns out that, in some cases, the aforementioned conditions can be removed completely (see $[70,77])$. In particular, the interface may be non-smooth or intersect the boundary of the domain. Motivated from this, we believe that one can show that $\eta_{\varepsilon} \rightarrow \sqrt{A^{+}}$ uniformly in $\mathbb{R}^{2}$, or at least in compact subsets of $\mathbb{R}^{2} \backslash \partial \mathscr{D}_{0}$, as $\varepsilon \rightarrow 0$, without assuming condition (7). (Here $\eta_{\varepsilon}$ denotes the minimizer of $G_{\varepsilon}$ or the ground state 
of $(21)_{-}$. ) In this regard, we refer to [56, Prop. 3.16] for a related result (for (22) with $q=2$ ).

Is there a " $\Gamma$-Convergence" theory [141] for (1), relating local minimizers of the limit functional (14) to local minimizers of (1), as $\varepsilon \rightarrow 0$ ?

We wonder if, besides the one-dimensional profile $V(x)$, there is a (genuine) two-dimensional one $v(x, z)$ that could be used in (60). In view of (61), (63), (64), and the matching conditions with $\sqrt{A^{+}}$, the profile $v$ should satisfy

$$
\begin{cases}\beta^{-2} v_{z z}+v_{x x}-v\left(v^{2}+x\right)=0, & (x, z) \in \mathbb{R}^{2}, \\ v-\sqrt{-x} \rightarrow 0 \text { as } x \rightarrow-\infty, & v \rightarrow 0 \text { as } x \rightarrow \infty\end{cases}
$$

with $v$ being $\ell_{\varepsilon} / \varepsilon^{\frac{2}{3}}$-periodic in $z$. As in [78] (see also [135]), after a simple transformation of the $z$ independent variable (the $x$ variable remains unchanged), abusing notation, we are led to the problem:

$$
\begin{aligned}
& v_{z z}+v_{x x}-v\left(v^{2}+x\right)=0, \quad(x, z) \in \mathbb{R}^{2}, \\
& v-\sqrt{-x} \rightarrow 0 \text { as } x \rightarrow-\infty, \quad v \rightarrow 0 \quad \text { as } x \rightarrow \infty,
\end{aligned}
$$

with $v$ being $\hat{\ell}_{\varepsilon} / \varepsilon^{\frac{2}{3}}$-periodic in $z$, where $\hat{\ell}_{\varepsilon}=\int_{0}^{\ell_{\varepsilon}} \beta_{\varepsilon}(\theta) d \theta$. The uniqueness of positive solutions to the above problem does not seem to follow from the approach of Brezis and Oswald, as in [125], since solutions are unbounded [compare with (211)]. In fact, we believe that entire solutions of the equation in (213) should satisfy the growth estimate $v(x, z)=O\left(|x|^{\frac{1}{2}}\right)$. Moreover, it is not clear how to adapt the uniqueness result of Brezis [42]. On the other hand, motivated from (30), it is natural to seek solutions such that

$$
v_{x}<0, \quad(x, z) \in \mathbb{R}^{2} .
$$

It is irresistible to compare problem (213), (215) with the famous De Giorgi conjecture for the Allen-Cahn equation (49), see for instance [87,107]. In this regard, it is interesting to investigate whether there are genuine two-dimensional solutions $v$ of problem (213), (215) or not. Note that solutions of the latter problem could be unbounded and, in particular, so could be $v_{z}$ (see [36,37] where a similar difficulty arises). We point out that the space dimension usually plays a very important role in these type of problems. Another direction could be to investigate the same question for stable solutions of (213), in the sense of (125), see also a remark in pg. 79 of the review article [72]. Actually, using the method of [87], one can show that any solution of (213), (215) is stable. A variation of these questions could be to consider problem (213)-(214), with the asymptotic behavior in (214) being uniform in $z \in \mathbb{R}$, along the lines of the so called Gibbons' conjecture (see also [36]). We remark that in this case, as in [107], the method of moving planes [108] can be applied to show that (215) holds.

Many recent papers deal with the study of semiclassical ground states for the focusing $(22)_{+}$, where the potential $W(\mathbf{y})-\lambda$ is positive but decays to zero, as $|\mathbf{y}| \rightarrow \infty$, at most like $|\mathbf{y}|^{-2}$ (see for instance [207] and the references therein). Can one study the defocusing case under analogous conditions? For $\varepsilon$ fixed, a related existence result may be found in [3]. 
Suppose, for simplicity purposes, that $W$ is an even, double-well, justify onedimensional potential (for example as in [124] or [139]), say $W(\mathbf{y})=\left(\mathbf{y}^{2}-1\right)^{2}$. What happens in the "degenerate case" when $\lambda$ equals the local maximum of $W$ ? Assuming that $W^{\prime \prime}(0)<0$, suitably blowing up at the origin, we expect that the fine behavior of solutions, as $\varepsilon \rightarrow 0$, near the origin should be determined by a solution of the problem:

$$
\left\{\begin{array}{l}
v^{\prime \prime}=v\left(v^{2}+\frac{W^{\prime \prime}(0)}{2} x^{2}\right)=0, \quad x \in \mathbb{R}, \\
v-\sqrt{\frac{-W^{\prime \prime}(0)}{2}}|x| \rightarrow 0 \quad \text { as }|x| \rightarrow \infty
\end{array}\right.
$$

Notice the similarities of the above problem with (B.233) below. Note also that in the case of a symmetric double-well potential (for any $\lambda$ ) formulas (210) do not hold due to tunneling effects, see for instance [104,121].

Finally, we believe that similar studies can be conducted in the case of the "exterior" problem, where $\lambda>W$ outside of a bounded domain and $\lambda<W$ in its interior. It is natural to assume that $\lambda-W \rightarrow c>0$ as $|y| \rightarrow \infty$, and consider the Gross-Pitaevskii equation (21)_ with boundary conditions $|u(\mathbf{y}, t)| \rightarrow c^{\frac{1}{q-1}}$ as $|\mathbf{y}| \rightarrow \infty$. The approach of [43] does not yield uniqueness of bounded ground states, namely solutions of the equation in (22)_ coupled with the aforementioned boundary conditions, if $N \geqq 3$ (compare with Remark 16), and one has to apply a sophisticated "squeezing" argument (see [84]). Let us mention that the stability of standing wave solutions of Gross-Pitaevskii equations, considered with nonzero boundary conditions at infinity, is a very active field of current research, see for instance [40].

Remark 29. It is worthwhile to mention that if $0 \leqq W(\mathbf{y})-\lambda \leqq c(1+|\mathbf{y}|)^{2+d}, \mathbf{y} \in$ $\mathbb{R}^{N}, N \geqq 3$, for some positive constants $c$, $d$, then (22) _ has infinitely many bounded solutions with positive lower bounds (see [136]).

Acknowledgments. The research leading to these results has received funding from the European Union's Seventh Framework Programme (FP7-REGPOT-2009-1) under Grant Agreement No. 245749. GK is supported under the "ARISTEIA I" Action of the "Operational programme education and lifelong learning" and is co-funded by the European Social Fund (ESF) and National Resources.

\section{Appendix A. A-Priori Estimates for the Linearized Operator Based on the Non-Degeneracy of the Inner Profile}

Here we will provide an alternative, more natural, proof of the important Proposition 5 that does not require knowledge of lower bound (71), whose proof is rather technical (recall Remark 11), but instead relies merely on the non-degeneracy of the Hastings-McLeod solution $V$. This proof has the flexibility to deal with problems where the corresponding inner profile $V$ is non-degenerate but the corresponding lower bound (71) may be hard to establish or fails (see Remark 34 below for an example where the latter case occurs). The latter situation certainly occurs when 
trying to construct unstable solutions (with respect to the parabolic dynamics) in related problems, see $[134,135]$. Let us also point out that it is not clear to us how to generalize the last part of the proof of (71) in [104] to the case of arbitrary power nonlinearity, as in Proposition 10 below (see also Remark 31 below).

PROOF OF PROPOSITION 5: Observe that it suffices to show the following a-priori estimate: There exists a constant $C$ such that if $\varepsilon$ is sufficiently small, $\varphi \in \mathscr{X} \cap C^{2+\alpha}\left(\mathbb{R}^{2}\right)$, and $f \in \mathscr{X} \cap C^{\alpha}\left(\mathbb{R}^{2}\right), 0<\alpha<1$, satisfy

$$
\mathscr{L}(\varphi)=f,
$$

then

$$
\|\varphi\|_{L^{\infty}\left(\mathbb{R}^{2}\right)} \leqq C\|f\|_{L^{\infty}\left(\mathbb{R}^{2}\right)} .
$$

To this end, as in the one-dimensional related problem treated in [191, Prop. 5.2], we will argue by contradiction. We remark that this indirect method has been used extensively in the study of elliptic singular perturbation problems involving transition and spike layers, see [20].

Firstly, note that without knowledge of the validity of (71), relation (106) would be

$$
3 u_{\text {ap }}^{2}-a\left(\varepsilon^{\frac{2}{3}} y\right) \geqq \begin{cases}c \varepsilon^{\frac{2}{3}}|x|, & \text { if } L \leqq|x| \leqq \delta \varepsilon^{-\frac{2}{3}}, \\ c+c\left|\varepsilon^{\frac{2}{3}} y\right|^{p}, & \text { otherwise, }\end{cases}
$$

for small $\varepsilon>0$, having increased the value of $L$ if necessary.

Suppose now that there exist sequences $\varepsilon_{n} \rightarrow 0, \varphi_{n} \in \mathscr{X} \cap C^{2+\alpha}\left(\mathbb{R}^{2}\right), f_{n} \in$ $\mathscr{X} \cap C^{\alpha}\left(\mathbb{R}^{2}\right)$ such that

$$
\left\{\begin{array}{l}
\mathscr{L}\left(\varphi_{n}\right)=\Delta \varphi_{n}-\varepsilon_{n}^{-\frac{2}{3}}\left(3 u_{\mathrm{ap}}^{2}-a\left(\varepsilon_{n}^{\frac{2}{3}} y\right)\right) \varphi_{n}=f_{n} \\
\left\|\varphi_{n}\right\|_{L^{\infty}\left(\mathbb{R}^{2}\right)}=1 \text { and }\left\|f_{n}\right\|_{L^{\infty}\left(\mathbb{R}^{2}\right)} \rightarrow 0
\end{array}\right.
$$

Keeping in mind that $\varphi_{n} \rightarrow 0$ as $|y| \rightarrow \infty$, we may assume that there exist $y_{n} \in \mathbb{R}^{2}$ such that, without loss of generality, we have

$$
\varphi_{n}\left(y_{n}\right)=1, \quad \nabla \varphi_{n}\left(y_{n}\right)=0, \quad \Delta \varphi_{n}\left(y_{n}\right) \leqq 0, \quad n \geqq 1 .
$$

From (A.218)-(A.219), we obtain that

$$
\operatorname{dist}\left(y_{n}, \tilde{\Gamma}_{\varepsilon_{n}}\right) \leqq C, \quad n \geqq 1,
$$

for some (generic) constant $C$ independent of $n \geqq 1$. Thus, abusing notation, we can write $y_{n}=\left(\beta^{-1}\left(\varepsilon_{n}^{\frac{2}{3}} z_{n}\right) x_{n}, z_{n}\right)$ with $\left|x_{n}\right| \leqq C, z_{n} \in\left[0, \varepsilon_{n}^{-\frac{2}{3}} \ell_{\varepsilon_{n}}\right)$. Therefore, passing to a subsequence, we may assume that

$$
x_{n} \rightarrow x_{*} \text { and } \varepsilon_{n}^{\frac{2}{3}} z_{n} \rightarrow z_{*} \in\left[0, \ell_{0}\right] .
$$

Recalling (56), (60), in terms of coordinates $(x, z)$, the equation in (A.219) takes the form

$$
\left(\varphi_{n}\right)_{z z}+\beta^{2}\left(\varepsilon_{n}^{\frac{2}{3}} z\right)\left(\varphi_{n}\right)_{x x}+\tilde{B}_{1}\left(\varphi_{n}\right)-\varepsilon_{n}^{-\frac{2}{3}}\left(3 u_{\text {ap }}^{2}-a\left(\varepsilon_{n}^{\frac{2}{3}} y\right)\right) \varphi_{n}=f_{n},
$$


in the neighborhood of the curve $\tilde{\Gamma}_{\varepsilon_{n}}$ described by $\left\{|x| \leqq \delta_{0} \varepsilon_{n}^{-\frac{2}{3}}, \quad z \in\left[0, \varepsilon_{n}^{-\frac{2}{3}} \ell_{\varepsilon_{n}}\right)\right\}$, where $\tilde{B}_{1}$ is the differential operator:

$$
\tilde{B}_{1}(\varphi)=\varepsilon^{\frac{4}{3}} \beta^{\prime \prime} \beta^{-1} x \varphi_{x}+\varepsilon^{\frac{4}{3}}\left(\beta^{\prime}\right)^{2} \beta^{-2} x^{2} \varphi_{x x}+2 \varepsilon^{\frac{2}{3}} \beta^{\prime} \beta^{-1} x \varphi_{x z}+B_{1}(\varphi),
$$

and $B_{1}$ is the differential operator in (57) where derivatives are expressed in terms of formulas (61) and $s$ replaced by $\beta^{-1} x$. By (68), the first relation in (90), and working as in (107), we obtain that

$$
3 u_{\text {ap }}^{2}-a\left(\varepsilon_{n}^{\frac{2}{3}} y\right)=\varepsilon_{n}^{\frac{2}{3}} \beta^{2}\left(\varepsilon_{n}^{\frac{2}{3}} z\right)\left(3 V^{2}(x)+x\right)+\mathscr{O}\left(\varepsilon_{n}^{\frac{4}{3}}\right)\left(x^{2}+1\right)
$$

uniformly in the region described below (A.223), as $n \rightarrow \infty$. Making use of (A.219)-(A.225), and a standard compactness argument, passing to a subsequence, we find that

$$
\varphi_{n} \rightarrow \phi \quad \text { in } C_{\mathrm{loc}}^{2}\left(\mathbb{R}^{2}\right)
$$

where $\phi$ satisfies

$$
\begin{aligned}
& \phi_{z z}+\beta_{0}^{2}\left(z_{*}\right) \phi_{x x}-\beta_{0}^{2}\left(z_{*}\right)\left(3 V^{2}(x)+x\right) \phi=0, \quad(x, z) \in \mathbb{R}^{2}, \\
& \|\phi\|_{L^{\infty}\left(\mathbb{R}^{2}\right)}=1 \quad\left(\phi\left(x_{*}, z_{*}\right)=1\right)
\end{aligned}
$$

Since

$$
3 V^{2}(x)+x \rightarrow \infty \text { as } x \rightarrow \pm \infty
$$

a standard barrier argument, as in (122), and elliptic estimates [109], yield that there exists a constant $C$ such that

$$
|\nabla \phi(x, z)|+|\phi(x, z)| \leqq C e^{-|x|}, \quad(x, z) \in \mathbb{R}^{2},
$$

(see also Lemma 7.3 in [80]). Let $\left(\mu_{1}, \psi_{1}\right)$ denote the principal eigenvalueeigenfunction pair of

$$
-\mathscr{M}(\psi)=-\psi^{\prime \prime}+\left(3 V^{2}(x)+x\right) \psi=\mu \psi, \quad \psi( \pm \infty)=0 .
$$

Without loss of generality, we may assume that $\psi_{1}$ is positive. Furthermore, we have

$$
\mu_{1}>0
$$

as testing against $V_{x}<0$ readily shows (see [191]). (By virtue of (A.228) and Theorem 10.7 in [121], the spectrum of $-\mathscr{M}$, in $L^{2}(\mathbb{R})$, consists of simple eigenvalues $\mu_{1}<\mu_{2}<\cdots$ with $\mu_{i} \rightarrow \infty$.) Now let

$$
\Phi(z)=\int_{-\infty}^{\infty} \phi(x, z) \psi_{1}(x) \mathrm{d} x,
$$


where $\phi$ is as in (A.226). From (A.226), (A.229), and (A.230) with $\psi=\psi_{1}, \mu=$ $\mu_{1}$, we calculate that

$$
\begin{aligned}
\Phi^{\prime \prime} & =\int_{-\infty}^{\infty} \phi_{z z}(x, z) \psi_{1}(x) \mathrm{d} x \\
& =\beta_{0}^{2}\left(z_{*}\right) \int_{-\infty}^{\infty}\left[-\phi_{x x}+\left(3 V^{2}(x)+x\right) \phi\right] \psi_{1}(x) \mathrm{d} x \\
& =\beta_{0}^{2}\left(z_{*}\right) \int_{-\infty}^{\infty} \phi\left[-\psi_{1}^{\prime \prime}+\left(3 V^{2}(x)+x\right) \psi_{1}\right] \mathrm{d} x \\
& =\mu_{1} \beta_{0}^{2}\left(z_{*}\right) \Phi
\end{aligned}
$$

and

$$
|\Phi| \leqq C, \quad z \in \mathbb{R}
$$

From (A.231), and the above two relations, it follows at once that $\Phi$ is identically zero, which contradicts the previous relation (A.227). Consequently, we have established the validity of the desired a-priori estimate (A.217).

The proof of the proposition is complete.

Remark 30. By adapting Lemma 5.3 of [191], we can show that relation (A.216) implies that $\|\varphi\|_{L^{2}\left(\mathbb{R}^{2}\right)} \leqq C\|f\|_{L^{2}\left(\mathbb{R}^{2}\right)}$ for some constant that is independent of $\varphi, f$ and small $\varepsilon>0$. In fact, as in [101, Thm. 1.2], we expect that more general a-priori estimates of the form $\|\varphi\|_{L^{p}\left(\mathbb{R}^{2}\right)} \leqq C_{p, q} \varepsilon^{\alpha_{p, q}}\|f\|_{L^{q}\left(\mathbb{R}^{2}\right)}$ hold true.

\section{Appendix B. Around the Hastings-McLeod Solution of the Painlevé-II Equation}

In this appendix we will provide a new proof of the existence of the HastingsMcLeod solution $V$ of the Painlevé-II equation (65). Moreover, we will establish various qualitative properties of the solution that are required for the singular perturbation analysis. In contrast to the original proof of Hastings and Mcleod [113] (see also [116]), where a shooting argument was employed, here we will use an upper and lower solution argument, which in principle is not restricted to ODE problems. Even though such an approach was successfully applied to this problem recently in [18], and very recently in [202], in our opinion our construction is more flexible and intuitive. The main advantage of our proof, compared to those of the latter references, is that, in the process, we also establish existence and uniqueness of a solution of problem (157), which seems to be a new and useful result (recall Remark 17). Although a sizable literature has been devoted to the study of the Painlevé equation (see $[99,129,149]$ ), we understand that the solution of this problem was not previously known.

Our choice of lower-solution is motivated from [71] where, in particular, the authors treat the problem

$$
u^{\prime \prime}=|u|^{p}-x, \quad x>0, \quad u(0)=0, \quad x^{-\frac{1}{p}} u(x) \rightarrow 1 \text { as } x \rightarrow \infty,
$$


where $p>1$ (recall the discussion in the third part of Section 1.2), see also [116, Sec. 3.2] and [122] for the case $p=2$ which is the Painlevé-I equation. On the other hand, our choice of upper-solution is motivated from [190] where, in particular, the authors treat the problem

$$
u^{\prime \prime}=u^{2}-x^{2}, \quad x \in \mathbb{R}, \quad u(x)-|x| \rightarrow 0 \quad \text { as }|x| \rightarrow \infty,
$$

see also Remark 38 below.

The notation in this appendix is independent of the rest of the paper.

As in [113], see also [32, pg. 200], we will prove the following more general result:

Proposition 10. Given $p>1$, there exists a unique nonnegative solution $U$ of

$$
-u^{\prime \prime}-x u+|u|^{p} u=0, \quad x \in \mathbb{R},
$$

such that

$$
u(x) \rightarrow 0 \text { as } x \rightarrow-\infty ; \quad u(x)-x^{\frac{1}{p}} \rightarrow 0 \text { as } x \rightarrow \infty .
$$

Furthermore, we have that $U^{\prime}>0$ in $\mathbb{R}$, and

$$
\begin{array}{r}
U(x)=\mathscr{O}\left(|x|^{-\frac{1}{4}} \mathrm{e}^{-\frac{2}{3}|x|^{\frac{3}{2}}}\right) \quad \text { as } x \rightarrow-\infty \\
U(x)-x^{\frac{1}{p}}=\mathscr{O}\left(x^{\frac{1-3 p}{p}}\right) \quad \text { as } x \rightarrow \infty .
\end{array}
$$

The solution $U$ is non-degenerate in the sense that there are no nontrivial bounded solutions of the problem

$$
\phi^{\prime \prime}-\left[(p+1) U^{p}-x\right] \phi=0, \quad x \in \mathbb{R} .
$$

Note that (37) falls in the above class of problems by means of the transformation $x \rightarrow-x$.

Remark 31. The results of this appendix can be used in extending the results of the current paper, and treat the defocusing (22) _ with arbitrary nonlinearity exponent $q>2$. In particular, the considered model of the latter equation, with nonlinearity exponents $7 / 3<q<3$ (in one space dimension and the model harmonic potential), is particularly relevant to the physics of BEC-BCS (Bardeen-Cooper-Schrieffer) transition in ultracold Fermi gases (see [206]).

As a stepping stone towards the proof of the above proposition, we will first prove the following result which, as we have already mentioned, is of interest in its own right. In particular, the solution $U_{+}$below will form the basis for our construction of a lower-solution to problem (B.234)-(B.235).

Proposition 11. Given $p>1$, there exists a unique solution $U_{+}$of the problem

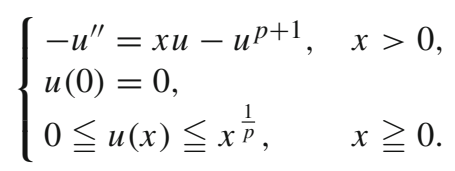


Furthermore, we have that $U_{+}^{\prime}(x)>0, x \geqq 0$, and

$$
U_{+}(x)-x^{\frac{1}{p}}=\mathscr{O}\left(x^{\frac{1-3 p}{p}}\right) \text { as } x \rightarrow \infty \text {. }
$$

Proof. It is easy to check that $x^{\frac{1}{p}}$ is an upper-solution of (B.237), while $\delta \chi_{[K, K+\pi]}$ $\sin (x-K)$ is a (weak) lower-solution provided that $K \geqq 2$ and $0<\delta \leqq 1$ (here $\chi$ denotes the characteristic function). (We refer the reader to [34] for more information on piecewise smooth weak upper/lower-solutions.) From now on we fix such a $K$, say $K=\pi$. Then, by a well known theorem [34], for every $0<\delta \leqq 1$, there exist solutions $u_{1}, u_{2}$ of (B.237) such that $\delta \chi_{[\pi, 2 \pi]} \sin (x-\pi) \leqq u_{1} \leqq u_{2} \leqq$ $x^{\frac{1}{p}}$, with the property that any solution of (B.237) such that $\delta \chi_{[\pi, 2 \pi]} \sin (x-\pi) \leqq$ $u \leqq x^{\frac{1}{p}}$, satisfies $u_{1} \leqq u \leqq u_{2}$. (Note that $u_{1}, u_{2}$ depend on $\delta$.)

For any nontrivial solution of (B.237) we have $u^{\prime \prime}=u\left(u^{p}-x\right) \leqq 0, x>0$. Thus $u^{\prime}$ is non-increasing. As a result, $u^{\prime} \rightarrow a$ as $x \rightarrow \infty$. Here $a$ may be minus infinity. We claim that $a=0$. In fact, if $a<0$, then $u(x)<0$ for $x$ large, which is a contradiction to $u \geqq 0$. If $a>0$, then $u(x) \geqq \frac{a}{2} x$ for $x>0$ large, which is a contradiction to $u(x) \leqq x^{\frac{1}{p}}$. Thus $a=0$. Consequently $u^{\prime} \geqq 0$. Actually, since $u^{\prime}$ cannot be constant over a nontrivial interval, we find that $u^{\prime}$ is decreasing and thus $u^{\prime}>0$.

In order to proceed further, we will show that problem (B.237) has a unique solution. Inspired by the work by BREZIS and OswaLd in [43] (see also [171, Sec. 8.5.2]), we will study the quotient of two solutions (for a different approach see Remark 32 below). Suppose that (B.237) has two solutions $\tilde{u}_{1}, \tilde{u}_{2}$. Then, we can find $0<\delta \leqq 1$ small such that $\delta \chi_{[\pi, 2 \pi]} \sin (x-\pi) \leqq \tilde{u}_{i} \leqq x^{\frac{1}{p}}, i=1,2$. Thus, from the previous discussion, we infer that $u_{1} \leqq \tilde{u}_{i} \leqq u_{2}, i=1,2$. We only need to prove that $u_{1}=u_{2}$. From (B.237), we find that

$$
\frac{u_{2}^{\prime \prime}}{u_{2}} \geqq \frac{u_{1}^{\prime \prime}}{u_{1}} .
$$

The above inequality implies that the function $u_{2}^{\prime} u_{1}-u_{2} u_{1}^{\prime}$ is non-decreasing, and so

$$
\left(u_{2}^{\prime} u_{1}-u_{2} u_{1}^{\prime}\right)(x) \geqq\left(u_{2}^{\prime} u_{1}-u_{2} u_{1}^{\prime}\right)(0)=0, \quad x>0,
$$

which in turn implies that the function $\frac{u_{2}}{u_{1}}$ is non-decreasing. Therefore, we get

$$
\frac{u_{2}(x)}{u_{1}(x)} \geqq \lim _{x \rightarrow 0^{+}} \frac{u_{2}(x)}{u_{1}(x)}=\frac{u_{2}^{\prime}(0)}{u_{1}^{\prime}(0)}, \quad x>0,
$$

by L'Hospital's rule (recall that $\left.u_{1}^{\prime}(0)>0\right)$. From $u_{2} \geqq u_{1}$ and $u_{1}(0)=u_{2}(0)=0$, we know that $u_{2}^{\prime}(0) \geqq u_{1}^{\prime}(0)$. Suppose that $u_{2}^{\prime}(0)>u_{1}^{\prime}(0)$. We have

$$
u_{1} \leqq \frac{u_{1}^{\prime}(0)}{u_{2}^{\prime}(0)} u_{2} \leqq \frac{u_{1}^{\prime}(0)}{u_{2}^{\prime}(0)} x^{\frac{1}{p}}, \quad x>0 .
$$


So

$$
u_{1}^{\prime \prime}=u_{1}\left(u_{1}^{p}-x\right) \leqq u_{1}\left[\left(\frac{u_{1}^{\prime}(0)}{u_{2}^{\prime}(0)}\right)^{p}-1\right] x \leqq-c x
$$

for $x>0$ large, and some constant $c>0$, since $u_{1}^{\prime}(0)<u_{2}^{\prime}(0)$ and $u_{1}^{\prime}>0$. It follows that $u_{1}^{\prime}<0$ for $x>0$ large. This is a contradiction to $u_{1}^{\prime}(x)>0, x \geqq 0$. We conclude that $u_{1}^{\prime}(0)=u_{2}^{\prime}(0)$, which gives $u_{1}=u_{2}$.

Let $u$ denote the unique solution of (B.237). Adapting an argument from [71], we will show that

$$
x^{-\frac{1}{p}} u(x) \rightarrow 1 \text { as } x \rightarrow \infty .
$$

From $u^{\prime}(\infty)=0$, we obtain

$$
\int_{0}^{\infty} u(x)\left(x-u^{p}(x)\right) \mathrm{d} x=-\int_{0}^{\infty} u^{\prime \prime}(x) \mathrm{d} x=u^{\prime}(0) .
$$

Hence, we can choose $x_{i} \rightarrow \infty$ such that $u\left(x_{i}\right)\left(x_{i}-u^{p}\left(x_{i}\right)\right) \rightarrow 0$ as $i \rightarrow \infty$, and recalling that $u^{\prime}>0$, we find that

$$
x_{i}-u^{p}\left(x_{i}\right) \rightarrow 0 \text { as } i \rightarrow \infty .
$$

Now, for any small $\theta>0$, we claim that

$$
u(x) \geqq(1-\theta) x^{\frac{1}{p}},
$$

for $x>0$ large. Suppose that the claim is not true. Then, there are $0<\theta<1$ and $\tilde{x}_{i} \rightarrow \infty$ such that

$$
u\left(\tilde{x}_{i}\right)<(1-\theta) \tilde{x}_{i}^{\frac{1}{p}} .
$$

It is easy to check that there is a $T>0$ large such that, for $x>T$, we have

$$
-\left((1-\theta) x^{\frac{1}{p}}\right)^{\prime \prime}<x(1-\theta) x^{\frac{1}{p}}-\left((1-\theta) x^{\frac{1}{p}}\right)^{p+1}
$$

By (B.240), we can choose a constant $\bar{T}>T$, such that $u(\bar{T})>(1-\theta) \bar{T}^{\frac{1}{p}}$. Define $v(x)=u(x)$ if $x \in[0, \bar{T}] ; v(x)=\max \left(u(x),(1-\theta) x^{\frac{1}{p}}\right)$ if $x \in[\bar{T}, \infty)$. Then $v$ is continuous, $0 \leqq v \leqq x^{\frac{1}{p}}$, and is a (weak) lower-solution of (B.237), in view of (B.242) and [34]. As a result, from [168, Thm. 2.10], problem (B.237) has a solution $u^{*}$ with $v \leqq u^{*} \leqq x^{\frac{1}{p}}$. On the other hand, since $v \geqq u$, and $u \neq v$ [by (B.241)], we find that $u^{*} \neq u$. This contradicts the uniqueness of the solutions of (B.237).

It remains to show the validity of estimate (B.238). We have

$$
u^{\prime \prime}=u \frac{\left(u^{p}-x\right)}{u-x^{\frac{1}{p}}}\left(u-x^{\frac{1}{p}}\right)=q(x)\left(u-x^{\frac{1}{p}}\right), \quad x>0,
$$


with

$$
\frac{q(x)}{x} \rightarrow p \text { as } x \rightarrow \infty
$$

by (B.239). (Note that, by the maximum principle, we get $u(x)<x^{\frac{1}{p}}, x>0$.) Let

$$
w=u-x^{\frac{1}{p}}, \quad x>0 .
$$

Then, from (B.243), we obtain that

$$
-w^{\prime \prime}+q(x) w-\frac{1}{p}\left(\frac{1}{p}-1\right) x^{\frac{1}{p}-2}=0, \quad x>0 .
$$

We claim that there exist $L, M>0$ sufficiently large such that, for every $\delta \in(0,1)$, the function

$$
\underline{w}(x)=-M x^{\frac{1}{p}-3}-\delta x^{\frac{1}{p}},
$$

is a lower-solution of (B.245) in $[L, \infty)$. Indeed, thanks to (B.244), for every $\delta \in(0,1)$, we find that

$$
\begin{aligned}
-\underline{w}^{\prime \prime} & +q(x) \underline{w}-\frac{1}{p}\left(\frac{1}{p}-1\right) x^{\frac{1}{p}-2} \\
\leqq & M\left(\frac{1}{p}-3\right)\left(\frac{1}{p}-4\right) x^{\frac{1}{p}-5}+\delta \frac{1}{p}\left(\frac{1}{p}-1\right) x^{\frac{1}{p}-2} \\
& -\frac{p}{2} M x^{\frac{1}{p}-2}-\frac{p}{2} \delta x^{\frac{1}{p}+1}-\frac{1}{p}\left(\frac{1}{p}-1\right) x^{\frac{1}{p}-2} \\
\leqq & M x^{\frac{1}{p}-5}\left[\left(\frac{1}{p}-3\right)\left(\frac{1}{p}-4\right)+\left(\frac{1}{M}-\frac{p}{2}\right) x^{3}\right]<0,
\end{aligned}
$$

for $x>L$, provided $M>\frac{4}{p}$ and $L$ is sufficiently large (independently of $M$ ). We fix such an $L>0$, and choose $M>\frac{4}{p}$ large, depending only on $L$, such that $\underline{w}(L)<w(L)$ for every $\delta \in(0,1)$. In view of (B.239), for every $\delta \in(0,1)$, we have

$\underline{w}(x)-w(x)=-M x^{\frac{1}{p}-3}-\delta x^{\frac{1}{p}}-\left(u-x^{\frac{1}{p}}\right) \leqq x^{\frac{1}{p}}\left(-\delta-\left(x^{-\frac{1}{p}} u-1\right)\right) \rightarrow-\infty$

as $x \rightarrow \infty$. Thus, by the maximum principle, we deduce that $\underline{w}(x) \leqq w(x), x \geqq L$, that is,

$$
u(x)-x^{\frac{1}{p}} \geqq-M x^{\frac{1}{p}-3}-\delta x^{\frac{1}{p}}, \quad x \geqq L, 0<\delta<1 .
$$

By letting $\delta \rightarrow 0$, we deduce that $u(x)-x^{\frac{1}{p}} \geqq-M x^{\frac{1}{p}-3}, x \geqq L$. The validity of (B.238) follows at once from this lower bound and the upper bound $u \leqq x^{\frac{1}{p}}$. The proof of the proposition is complete. 
Remark 32. An alternative way to establish uniqueness for (B.237) is the following: Suppose that $V_{+}$solves (B.237). Then, it is easy to see that $\lambda V_{+}, \lambda \geqq 1$, is a family of upper solutions of (B.237) such that $\lambda V_{+}(0)=U_{+}(0)=0$, and $\lambda V_{+}-U_{+} \rightarrow \infty$ as $x \rightarrow \infty$ if $\lambda>1$. Moreover, since $V_{+}^{\prime}(0)>0$, we have that $\lambda V_{+}>U_{+}$in $(0, \infty)$ if $\lambda \gg 1$. Therefore, by Serrin's sweeping technique (see $\left[184\right.$, pg. 40]), we get $V_{+} \geqq U_{+}$in $[0, \infty)$. Similarly, we can show that $V_{+} \leqq U_{+}$ in $[0, \infty)$. Consequently, we get that $V_{+} \equiv U_{+}$.

Remark 33. In relation to the problems mentioned in the third part of Section 1.2, it would be of interest to generalize Proposition 11 in the following direction: study solutions $u: \mathbb{R}^{2} \rightarrow \mathbb{C}$ (if they exist) of the problem

$$
\begin{cases}\Delta u+\left(|\mathbf{y}|-|u|^{p}\right) u=0, & \mathbf{y} \in \mathbb{R}^{2}, \\ u(\mathbf{0})=0 ; & |\mathbf{y}|^{-\frac{1}{p}}|u| \rightarrow 1 \text { as }|\mathbf{y}| \rightarrow \infty\end{cases}
$$

We cannot resist comparing the above problem with the well known

$$
\begin{cases}\Delta u+\left(1-|u|^{2}\right) u=0, & \mathbf{y} \in \mathbb{R}^{2}, \\ u(\mathbf{0})=0 ; & |u| \rightarrow 1 \text { as }|\mathbf{y}| \rightarrow \infty\end{cases}
$$

see $[120,170]$, and the references in the research monographs $[39,171,183]$.

The following proposition plays an important role in relation with Remark 17.

Proposition 12. The solution $U_{+}$of (B.237) is non-degenerate in the sense that there are no nontrivial bounded solutions of the problem

$$
\phi^{\prime \prime}-\left[(p+1) U_{+}^{p}-x\right] \phi=0, \quad x>0, \phi(0)=0 .
$$

Proof. Suppose that there exists a nontrivial bounded solution $\phi$ of (B.246). The fact that $(p+1) U_{+}^{p}-x \rightarrow \infty$ as $x \rightarrow \infty$, easily implies that $\phi$ has a finite number of zeros in $[0, \infty)$, all of them simple, and that $|\phi|,\left|\phi^{\prime}\right|$ decay to zero super-exponentially as $x \rightarrow \infty$. Let $r_{0} \geqq 0$ be the largest zero of $\phi$. Without loss of generality, we may assume that $\phi^{\prime}\left(r_{0}\right)>0$. Differentiating (B.237), multiplying the resulting identity by $\phi$, then multiplying (B.246) by $U_{+}^{\prime}$, subtracting, and integrating by parts over $\left(r_{0}, \infty\right)$, we readily arrive at

$$
U_{+}^{\prime}\left(r_{0}\right) \phi^{\prime}\left(r_{0}\right)=-\int_{r_{0}}^{\infty} U_{+} \phi \mathrm{d} x .
$$

However, this is a contradiction to $U_{+}^{\prime}>0, \phi^{\prime}\left(r_{0}\right)>0$, and $\phi(x)>0, x>r_{0}$. The proof of the proposition is complete.

Remark 34. Note that, since $U_{+}(0)=0$ and $p>1$, the potential $(p+1) U_{+}^{p}-x$ of the linear operator in the righthand side of (B.246) is negative for small $x>0$.

We can now give the PROOF OF PROPOSITION 10: Let $U_{+}$be as in Proposition 11, it is clear that

$$
\underline{u}(x)= \begin{cases}U_{+}(x), & x \geqq 0, \\ 0, & x \leqq 0\end{cases}
$$


is a (weak) lower-solution of (B.234)-(B.235).

Next we will construct an upper-solution of (B.234)-(B.235), for a different construction we refer the interested reader to Remark 40 below. Let

$$
u_{0}(x)= \begin{cases}x^{\frac{1}{p}}, & x \geqq 0 \\ 0, & x \leqq 0\end{cases}
$$

Then, fix a continuous function $\phi \geqq 0$ such that $u_{0}+\phi \in C^{2}(\mathbb{R})$ and $\phi(x)=0$ if $|x| \geqq 1$. Let $\mu_{1}>0, \psi_{1}>0$ denote the principal eigenvalue and the corresponding $L^{\infty}$-normalized eigenfunction of

$$
-\psi^{\prime \prime}+\left((p+1) u_{0}^{p}-x\right) \psi=\mu \psi, \quad \psi \in L^{2}(\mathbb{R}) .
$$

Such $\mu_{1}, \psi_{1}$ exist, since the potential

$$
Q(x) \equiv(p+1) u_{0}^{p}-x= \begin{cases}p x, & x \geqq 0, \\ -x, & x \leqq 0,\end{cases}
$$

clearly satisfies $\inf _{x \in \mathbb{R}} Q(x)<\liminf _{x \rightarrow \pm \infty} Q(x)$, see [178] (see also [121, Thm. 10.7]). Furthermore, $\psi_{1},\left|\psi_{1}^{\prime}\right|,\left|\psi_{1}^{\prime \prime}\right|$ decay to zero super-exponentially as $|x| \rightarrow$ $+\infty$. More precisely, there exist constants $c_{ \pm}$and $x_{-}<0, x_{+}>0$ such that

$$
\psi_{1}(x) \sim c_{ \pm} \frac{\exp \left\{-\left|\int_{x_{ \pm}}^{x} \sqrt{Q(t)-\mu_{1}} \mathrm{~d} t\right|\right\}}{\left[Q(x)-\mu_{1}\right]^{\frac{1}{4}}} \text { as } x \rightarrow \pm \infty
$$

see [64, Chap. IV, Thm. 14] and [16, Appx. A]. We can now define our upper solution for (B.234)-(B.235) as

$$
\bar{u}=u_{0}+\phi+M \zeta_{D} \psi_{1}
$$

with $M, D>1$ large constants to be chosen, and $\zeta_{D}(x)=z(x-D)$, where $z \in C^{\infty}(\mathbb{R})$ is such that $z=1, t \leqq 0 ; z=0, t \geqq 1 ; z^{\prime}<0, t \in(0,1)$. We proceed in verifying that $\bar{u}$ is indeed an upper solution. In $[0,1], \bar{u}=\left(u_{0}+\phi\right)+M \psi_{1}$ and

$$
-\bar{u}^{\prime \prime}-x \bar{u}+\bar{u}^{p+1} \geqq-C M+c M^{p+1}>0,
$$

provided $M>0$ is sufficiently large $(C, c>0$ are independent of large $M)$. We fix such an $M>0$. In [1,D], we have $\bar{u}=u_{0}+M \psi_{1}$ and

$$
\begin{gathered}
-\bar{u}^{\prime \prime}-x \bar{u}+\bar{u}^{p+1}=-u_{0}^{\prime \prime}-M \psi_{1}^{\prime \prime}-x u_{0}-M x \psi_{1}+\left(u_{0}+M \psi_{1}\right)^{p+1} \\
\geqq-M \psi_{1}^{\prime \prime}-x u_{0}-M x \psi_{1}+u_{0}^{p+1}+(p+1) u_{0}^{p} M \psi_{1}=M \mu_{1} \psi_{1}>0 .
\end{gathered}
$$

In $[D, D+1]$, we have $\bar{u}=u_{0}+M \zeta_{D} \psi_{1}$ and

$$
\begin{aligned}
-\bar{u}^{\prime \prime}-x \bar{u}+\bar{u}^{p+1} & =-u_{0}^{\prime \prime}-M\left(\zeta_{D} \psi_{1}\right)^{\prime \prime}-x u_{0}-M x \zeta_{D} \psi_{1}+\left(u_{0}+M \zeta_{D} \psi_{1}\right)^{p+1} \\
& \geqq-\frac{1}{p}\left(\frac{1}{p}-1\right) x^{\frac{1}{p}-2}-C e^{-x}-x u_{0}+u_{0}^{p+1} \\
& =-\frac{1}{p}\left(\frac{1}{p}-1\right) x^{\frac{1}{p}-2}-C e^{-x}>0
\end{aligned}
$$


provided $D$ is chosen large $(C>0$ is independent of $D)$. In $[D+1,+\infty)$, we plainly have $\bar{u}=x^{\frac{1}{p}}$. Analogous calculations also hold in $(-\infty, 0]$. Consequently, the function $\bar{u}$ is an upper-solution of (B.234)-(B.235).

It follows from [168, Thm. 2.10] as before that there exists a solution of (B.234) such that $\underline{u} \leqq u \leqq \bar{u}$. The second estimate in (B.236) follows at once from (B.238) and (B.247); the first one follows from the fact that, for every solution of (B.234) that tends to zero as $x \rightarrow-\infty$, there exists some constant $c>0$ such that

$$
u(x) \sim c \operatorname{Ai}(-x) \text { as } x \rightarrow-\infty,
$$

[recall the discussion leading to (75)], and (74).

We will show that $u^{\prime}>0$. We follow [18]. Since $0 \leqq u \leqq x^{\frac{1}{p}}$ for $x \geqq D+1$, as in the proof of Proposition 11, we obtain that $u^{\prime} \rightarrow 0$ as $x \rightarrow \infty$. Moreover, it is easy to show that $u^{\prime} \rightarrow 0$ super-exponentially as $x \rightarrow-\infty$. Since $u$ is strictly positive (by the maximum principle), in view of (B.234), we can write

$$
\left(\frac{u^{\prime \prime}}{u}-u^{p}\right)^{\prime}=-1,
$$

that is,

$$
v^{\prime \prime}-\frac{1}{u} v v^{\prime}-p u^{p} v=-u, \quad \text { where } v=u^{\prime} .
$$

Since $v \rightarrow 0$ as $|x| \rightarrow \infty$, it follows that if $v$ is not strictly positive, then there exists $x_{0}$ such that

$$
v\left(x_{0}\right) \leqq 0, \quad v^{\prime}\left(x_{0}\right)=0, \quad v^{\prime \prime}\left(x_{0}\right) \geqq 0,
$$

but this is impossible because (B.253) implies

$$
v^{\prime \prime}\left(x_{0}\right)-p u^{p}\left(x_{0}\right) v\left(x_{0}\right)=-u\left(x_{0}\right)<0,
$$

and therefore we conclude that $u^{\prime}$ is strictly positive. The same conclusion can also be derived by adapting an argument from [202], that is, applying the maximum principle to the function $\frac{u^{\prime}}{u}$ (keep in mind the second identity in (79)). A more PDE approach is to apply the moving plane method [108], starting from $-\infty$, as in [107]. Uniqueness (of nonnegative solutions) for the problem (B.234)-(B.235) can be established in a similar manner as we did in Proposition 11 for the problem (B.237). Again we suppose that there exist two distinct non-negative solutions $u_{1}, u_{2}$ of (B.234)-(B.235). By the strong maximum principle, we deduce that both are strictly positive. Hence, there is some small $\delta>0$ such that $u_{i}(x) \geqq \delta \chi_{[\pi, 2 \pi]} \sin (x-\pi)$, $i=1,2$, for every $x \in \mathbb{R}$. Recall that the function in the righthand side is a lowersolution of (B.234)-(B.235). Moreover, both $u_{i}, i=1$, 2, will eventually lie below the graph of $x^{\frac{1}{p}}$ (note that $u-x^{\frac{1}{p}}$ is strictly convex as long as it is nonnegative). Thus, by virtue of (74), (B.249), (B.250) and (B.252), we can choose sufficiently large numbers $D, M$ such that $u_{i}(x) \leqq \bar{u}(x), i=1,2$, for every $x \in \mathbb{R}$. [This is a fine point that was not present in the uniqueness proof for (B.237).] Recall that the function in the righthand side is an upper-solution of (B.234)-(B.235). 
Consequently, we may assume that $u_{1}(x) \leqq u_{2}(x), x \in \mathbb{R}$, and it is easy to see that $\frac{u_{2}}{u_{1}}$ is non-decreasing in $\mathbb{R}$. On the other hand, it follows from the second relation in (B.235) that $\frac{u_{2}}{u_{1}} \rightarrow 1$ as $x \rightarrow \infty$. So, we get that $u_{2} \leqq u_{1}$ in $\mathbb{R}$ which is a contradiction.

Finally, the non-degeneracy of $U$ can be derived as in Proposition 12 for the nondegeneracy of $U_{+}$.

The proof of the proposition is complete.

Remark 35. The fact that problem (B.234)-(B.235) has a unique solution, which is a stronger result, has been proven in [113].

In contrast, problem (B.232) with $p=2$, has exactly two solutions. The existence of two solutions has been established by Holmes and Spence [122] by a shooting argument (and in [71] for any $p>1$, via the method of upper/lower solutions and variational arguments, perhaps unaware of [122]), where the authors also conjectured that these solutions were indeed the only ones. Their conjecture was settled, to the affirmative, by Hastings and Troy [114]. However, their proof was, as we discover now (almost 25 years later!), much more complicated than necessary, and relied on some four decimal point numerical calculations. Motivated by an idea of ours from [135], where problem (B.233) was shown to have exactly two solutions, we can give a truly simple proof of the uniqueness result of [114] as follows. We know from [71,122] that problem (B.232) with $p=2$ has a unique increasing solution $\mathscr{U}_{+}$. Let $\tilde{\mathscr{U}}$ be any other solution, and let $\eta=\mathscr{U}_{+}-\tilde{\mathscr{U}}$. By an easy calculation, and the maximum principle, we find that $\eta$ has to be a positive solution of

$$
\eta^{\prime \prime}-2 \mathscr{U}_{+}(x) \eta+\eta^{2}=0, \quad x>0, \quad \eta(0)=0, \quad \eta(x) \rightarrow 0 \quad \text { as } x \rightarrow \infty .
$$

The key observation now is that the solution $\eta$ furnishes an odd standing wave solution of a focusing NLS equation of the form $(21)_{+}$, with $N=1, q=2$. Thankfully, in the last years a lot of research and efforts of many authors have been devoted to the uniqueness of ground states of radially symmetric focusing nonlinear Schrödinger equations with non-decreasing potential (in $r>0$ ) and power nonlinearity, considered in the whole space, in a ball, or an annulus (see [48] for the state of the art.) The problem of uniqueness of $\eta$ resembles more the case of the annulus and, having all those tools at our disposal which were not available at the time that [114] was written, we can infer that uniqueness as well as nondegeneracy of a positive solution $\eta$ of (B.254) follow directly from [91, Thm. 1.2]. (In the latter reference, it was assumed that the potential is strictly positive but it is easy to check that their proof works equally well for the case at hand, see also $[48,132]$.) The non-degeneracy property of the solution $\tilde{\mathscr{U}}$, which follows readily, is a new result and, in the context of the original singular perturbation problem [197] (see also [71,73]) is more useful than uniqueness (recall Remark 11).

To the best of our knowledge, the similarities between the singularly perturbed (multi-dimensional) elliptic problem in [71], arising from the study of the LazerMckenna conjecture, and the one-dimensional one in [114,122,197], arising from 
the problem of vertical flow of an internally heated Boussinesq fluid with viscous dissipation and pressure work, were previously unknown.

The proof of the Lazer-Mckenna conjecture in [71] consists of constructing solutions of the problem with arbitrary many (clustering) sharp downward spikes on top of a positive minimizer of the corresponding energy functional, as the small parameter $\varepsilon>0$ tends to zero. The aforementioned minimizer has a corner layer, along the boundary of the domain, whose profile is described by the positive solution of (B.232). It is our hope that the techniques of the present paper, together with those already developed in $[78,160]$, can be used to construct new solutions, having corner layer profile described by the unstable solution of (B.232) (is this unique and non-degenerate for $p \neq 2$ ?), at least when $\varepsilon$ stays away from certain critical values $\varepsilon_{1}>\varepsilon_{2}>\cdots>\varepsilon_{i} \rightarrow 0$ (see [135] for a related problem). These solutions would be slightly negative in a small ( $\varepsilon$-dependent) neighborhood of the boundary, with their Morse index diverging as $\varepsilon \rightarrow 0$ (away from the critical numbers). Then, one could use the techniques of $[85,200]$ in order to add downward spikes on top of that unstable solution. One may even be able to prove the existence of arbitrary many solutions, as $\varepsilon \rightarrow 0$, which was the original assertion of the Lazer-Mckenna conjecture settled in [71], just by the fact that the solution's Morse index diverges [recall also the discussion following (210)]. This would constitute a proof of the Lazer-Mckenna conjecture, as treated in $[71,73]$, that is valid even for supercritical exponents.

Remark 36. The related boundary value problem

$$
u_{x x}=u\left(u^{2}-x\right), \quad x>0 ; \quad u_{x}(0)=0, \quad u-\sqrt{x} \rightarrow 0 \text { as } x \rightarrow \infty,
$$

arises in the study of the superheating field attached to a semi-infinite superconductor, for the construction of a family of approximate solutions of the GinzburgLandau system via the procedure of (formally) matching inner and outer solutions (see [58,117]). Existence for the problem (B.255) has been established by shooting arguments in [117], and by topological ones in [172]. We can give a new proof of their results, valid for any power nonlinearity (as in Proposition 11), by slightly modifying the above proof of Proposition 10 as follows: One still uses $U_{+}$as a lower solution of (B.255) (it is a solution and $U_{+}^{\prime}(0)>0$, see [34]); however in the construction of the upper solution we have to be careful to chose the function $\phi$ such that $\left(u_{0}+\phi\right)_{x}(0) \leqq 0$ (and afterwards the principal eigenfunction subject to Neumann boundary conditions at $x=0$ ). It was shown in [112] that, without any assumptions at infinity, problem (B.255) has exactly one global positive solution. As a matter of fact, we expect that an analogous property holds true for the problem (B.237), see also [42] for a related result concerning (B.233).

Remark 37. Since $p>1$, the solutions that we have constructed in Propositions 10,11 have infinite energy (more precisely, their derivative does not belong in $L^{2}(0, \infty)$ ). Nevertheless, we believe that one can also establish existence (and further characterize the solutions) for problems (B.234)-(B.235), (B.237), and (B.255) by minimizing a suitable re-normalized energy functional, as in [170], or 
minimizing the standard energy functional of (B.234) in a large interval $[-R, R]$ with boundary conditions $u(-R)=0 ; u(R)=R^{\frac{1}{p}}$, and then letting $R \rightarrow \infty$ (see $[37,50,82]$ for some related situations). Similarly for the other cases.

Remark 38. If $0<p \leqq 1$, then there exists a unique solution of (B.234)-(B.235) such that $u>u_{0}$, where $u_{0}$ is as in (B.248). We cannot resist to give a short proof of this, based on [134]. If $0<p<1$, it is easy to see that $u_{0}$ is a lower solution, while $u_{0}+\varphi$, with $\varphi>0$ defined by

$$
-\varphi_{x x}+\left[(p+1) u_{0}^{p}-x\right] \varphi=\left(u_{0}\right)_{x x} \geqq 0, \quad \varphi( \pm \infty)=0,
$$

is an upper solution of (B.234)-(B.235). If $p=1$, then $u_{0}$ is a weak lower solution, while $u_{0}+\phi$, with $\phi>0$ the unique continuous solution of

$$
-\phi_{x x}+\left[(p+1) u_{0}^{p}-x\right] \phi=0, \quad \phi_{x}\left(0^{-}\right)-\phi_{x}\left(0^{+}\right)=1, \quad \phi( \pm \infty)=0,
$$

is an upper solution of (B.234)-(B.235) [recall (B.249)]. Existence of the desired solution follows at once. Uniqueness follows simply by taking the difference of the equations satisfied by two pairs of solutions.

The case $p=1$, which is equivalent to (B.233), has received considerable attention lately, mainly since it appears in the study of the spatial segregation limit of competitive systems $[63,127]$ (see also [123,190]). Interestingly enough, we have noticed that problem (B.233) also describes the corner layer profile of solutions in the paper [115] by Hastings and McLeod, in the case where the constant $c$ therein, which arises from an integration, is chosen to be zero rather than strictly positive.

Remark 39. Similar results should also hold true for the equation

$$
u^{\prime \prime}+x|x|^{s} u-|u|^{p} u=0, \quad x \in \mathbb{R},
$$

where $p, s>0$.

Remark 40. As we have already seen in Remark 38, there exists a unique solution $U>\max \{0, x\}$ of the problem

$$
u^{\prime \prime}=p u(u-x)=0, \quad x \in \mathbb{R}
$$

such that $U \rightarrow 0$ as $x \rightarrow-\infty ; U-x \rightarrow 0$ as $x \rightarrow \infty$. (We have found out in [135], by arguing as in Remark 35 above, that there exists exactly one more solution $U_{-}$of (B.256) which satisfies the boundary conditions, and in fact $U_{-}<\max \{0, x\}$.) Actually, the solution $U$ is the unique (global) solution of (B.256) such that $u \geqq \frac{x}{2}, x \in \mathbb{R}$. This follows at once from the fact that the equation in (B.233) has a unique nonnegative solution, see [42]. Moreover, since $p>1$, it is easy to see that the function $U^{\frac{1}{p}}$ is an upper-solution of (B.234)-(B.235). 


\section{References}

1. Ablowitz, M.J., Prinari, B., Trubatch, A.D.: Discrete and Continuous Nonlinear Schrödinger Systems. Cambridge University Press, Cambridge, (2004)

2. Achilleos, V., Theocharis, G., Kevrekidis, P.G., Karachalios, N.I., Diakonos, F.K., FrantZeSKaKIS, D.J.: Stationary states of a nonlinear Schrödinger lattice with a harmonic trap. J. Math. Phys. 52, 092701 (2011)

3. Afrouzi, G.A., Brown, K.J.: On a diffusive logistic equation. J. Math. Anal. Appl. 225, 326-339 (1998)

4. Aftalion, A., Rivière, T.: Vortex energy and vortex bending for a rotating BoseEinstein condensate. Phys. Rev. A 64, 043611 (2001)

5. Aftalion, A., Du, Q.: Vortices in a rotating Bose-Einstein condensate: critical angular velocities and energy diagrams in the Thomas-Fermi regime. Phys. Rev. A 64, 063603 (2001)

6. Aftalion, A., Du, Q., Pomeau, Y.: Dissipative flow and vortex shedding in the Painlevé boundary layer of a Bose-Einstein condensate. Phys. Rev. Lett. 91, 090407 (2003)

7. Aftalion, A., Blanc, X.: Existence of vortex free solutions in the Painlevé boundary layer of a Bose Einstein condensate. J. Math. Pures Appl. 83, 765-801 (2004)

8. Aftalion, A., Alama, S., Bronsard, L.: Giant vortex and the breakdown of strong pinning in a rotating Bose-Einstein condensate. Arch. Ration. Mech. Anal. 178, 247$286(2005)$

9. Aftalion, A.: Vortices in Bose Einstein Condensates. Birkhäuser Boston, Boston, (2006)

10. Aftalion, A., Jerrard, R.L., Royo-Letelier, J.: Non-existence of vortices in the small density region of a condensate. J. Funct. Anal. 260, 2387-2406 (2011)

11. Alama, S., Tarantello, G.: On the solvability of a semilinear elliptic equation via an associated eigenvalue problem. Math. Z. 221, 467-493 (1996)

12. Alama, S., Bronsard, L.: Pinning effects and their breakdown for a Ginzburg-Landau model with normal inclusions. J. Math. Phys. 46, 095102 (2005)

13. Alama, S., Bronsard L., Montero, J.A.: Vortices for a rotating toroidal BoseEinstein condensate. Arch. Ration. Mech. Anal. 187, 481-522 (2008)

14. Alama, S., Bronsard, L., Millot, V.: Gamma-convergence of 2D Ginzburg-Landau functionals with vortex concentration along curves. J. Anal. Math. 114, 341-391 (2011)

15. Alessio, F., Calamai, A., Montecchiari, P.: Saddle-type solutions for a class of semilinear elliptic equations. Adv. Differ. Equ. 12, 361-380 (2007)

16. Alfimov, G.L., Zezyulin, D.A.: Nonlinear modes for the Gross-Pitaevskii equation-a demonstrative computation approach. Nonlinearity 20, 2075-2092 (2007)

17. Alikakos, N.D., Fusco, G., Stefanopoulos, V.: Critical spectrum and stability of interfaces for a class of reaction-diffusion equations. J. Differ. Equ. 126, 106-167 (1996)

18. Alikakos, N.D., Bates, P.W., Cahn, J.W., Fife, P.C., Fusco, G., Tanoglu, G.B.: Analysis of the corner layer problem in anisotropy. Discrete Contin. Dyn. Syst. 6, 237-255 (2006)

19. Ambrosetti, A., Prodi, G.: A Primer of Nonlinear Analysis. Cambridge University Press, Cambridge, (1995)

20. Ambrosetti, A., Malchiodi, A.: Perturbation Methods and Semilinear Elliptic Problems. Birkhäuser Verlag, Basel, 2006

21. Ambrosetti, A., Malchiodi, A.: Nonlinear Analysis and Semilinear Elliptic Problems. Cambridge University Press, Cambridge, (2007)

22. André, N., Bauman, P., Phillips, D.: Vortex pinning with bounded fields for the Ginzburg-Landau equation. Ann. Inst. H. Poincaré Anal. Non Linéaire 20, 705-729 (2003)

23. André, N., Shafrir, I.: Minimization of a Ginzburg-Landau type functional with nonvanishing Dirichlet boundary condition. Calc. Var. 7, 1-27 (1998) 
24. Andrews, B. Clutterbuck, J.: Proof of the fundamental gap conjecture. J. Am. Math. Soc. 24, 899-916 (2011)

25. Baldo, S., Orlandi, G., Jerrard, R., Soner, M.: Vortex density models for superconductivity and superfluidity (2010, preprint)

26. Band, Y.B., Towers, I., Malomed, B.A.: Unified semiclassical approximation for Bose-Einstein condensates: application to a BEC in an optical potential. Phys. Rev. A 67, 023602 (2003)

27. Bartsch, T., Wang, Z.-Q., Willem, M.: The Dirichlet problem for superlinear elliptic equations. In: Handbook of Differential Equations: Stationary Partial Differential Equations, vol. II, pp. 1-71. Elsevier, 2005

28. Bates, P.W., Jones, C.K.R.T.: Invariant manifolds for semilinear partial differential equations. Dyn. Rep. 2, 1-38 (1989)

29. BAO, W., Du, Q.: Computing the ground state solution of Bose-Einstein condensates by a normalized gradient flow. SIAM J. Sci. Comput. 25, 1674-1697 (2004)

30. BAO, W.: Ground states and dynamics of rotating Bose-Einstein condensates. In: CERCignani, C., Gabetta, E. (eds) Transport Phenomena and Kinetic Theory. Birkhaüser, Boston, (2007)

31. BaO, W.: Some questions related to NLS. Slides from a talk (2012). Available online at http://www.acmac.uoc.gr/SMAW2012/talks

32. Bender, C.M., Orszag, S.A.: Advanced Mathematical Methods for Scientists and Engineers: Asymptotic Methods and Perturbation Theory. Springer, New York, (1999)

33. Benoît, E. (Ed.): Dynamic Bifurcations. Lecture Notes in Mathematics, vol. 1493. Springer, Heidelberg, (1991)

34. Berestycki, H., Lions, P.L.: Some applications of the method of super and subsolutions. In: Bardos, C., Lasry, J.M., Schatzman, M (eds.) Bifurcation and nonlinear eigenvalue problems. Lecture Notes in Mathematics, vol. 782, pp. 16-41. Springer, Heidelberg (1980)

35. Berestycki, H., Nirenberg, L., Varadhan, S.R.S.: The principal eigenvalue and maximum principle for second-order elliptic operators in general domains. Commun. Pure Appl. Math. 47, 47-92 (1994)

36. BerestycKi, H., Lin, T.C., Zhao, C.Y.: On phase-separation model: asymptotics and qualitative properties. Arch. Ration. Mech. Anal. 208, 163-200 (2013)

37. Berestycki, H., Terracini, S., Wang, K., Wei, J.: On entire solutions of an elliptic system modeling phase separations. Adv. Math. 243, 102-126 (2013)

38. Béthuel, F., Brezis, H., HÉlein, F.: Asymptotics for the minimization of a GinzburgLandau functional. Calc. Var. 1, 123-148 (1993)

39. Béthuel, F., Brezis, H., HÉLeIn, F.: Ginzburg-Landau Vortices. Birkhäuser, Boston, (1994)

40. Béthuel, F., Gravejat, P., Saut, J.-C., Smets, D.: Orbital stability of the black soliton to the Gross-Pitaevskii equation. Indiana Univ. Math. J. 57, 2611-2642 (2008)

41. Boscolo, S., Turitsyn, S.K., Novokshenov, V.Yu., NiJhof, J.H.: Self-similar parabolic optical solitary waves. Theor. Math. Phys. 133, 1647-1656 (2002)

42. Brezis, H.: Semilinear equations in $\mathbb{R}^{n}$ without conditions at infinity. Appl. Math. Optim. 12, 271-282 (1984)

43. Brezis, H., Oswald, L.: Remarks on sublinear elliptic equations. Nonlinear Anal. 10, 55-64 (1986)

44. Brunovský, P., Fiedler, B.: Number of zeros on invariant manifolds in reactiondiffusion equations. Nonlinear Anal. 10, 179-193 (1986)

45. Butuzov, V.F., Nefedov, N.N., Schneider, K.R.: Singularly perturbed boundary value problems for systems of Tichonov's type in case of exchange of stabilities. $J$. Differ. Equ. 159, 427-446 (1999)

46. Butuzov, V.F., Nefedov, N.N., Schneider, K.R.: Singularly perturbed elliptic problems in the case of exchange of stabilities. J. Differ. Equ. 169, 373-395 (2001)

47. ByeOn, J., Wang, Z.-Q.: Standing waves with a critical frequency for nonlinear Schrödinger equations. Arch. Ration. Mech. Anal. 165, 295-316 (2002) 
48. Byeon, J., Oshita, Y.: Uniqueness of standing waves for nonlinear Schrödinger equations. Proc. R. Soc. Edinb. Sect. A 138, 975-987 (2008)

49. Cabré, X., Capella, A.: On the stability of radial solutions of semilinear elliptic equations in all of $\mathbb{R}^{n}$. C. R. Acad. Sci. Paris, Ser. I 338, 769-774 (2004)

50. Cabré, X., Terra, J.: Saddle-shaped solutions of bistable diffusion equations in all of $\mathbb{R}^{2 m}$. J. Eur. Math. Soc. 11, 819-843 (2009)

51. Cabré, X., Terra, J.: Qualitative properties of saddle-shaped solutions to bistable diffusion equations. Commun. Partial Differ. Equ. 35, 1923-1957 (2010)

52. CABré, X.: Uniqueness and stability of saddle-shaped solutions to the Allen-Cahn equation. J. Math. Pures Appl. 98, 239-256 (2012)

53. CAfFarelli, L.A., RoQueJoffre, J.-M.: Uniform Hölder estimates in a class of elliptic systems and applications to singular limits in models for diffusion flames. Arch. Ration. Mech. Anal. 183, 457-487 (2007)

54. Caffarelli, L.A., Lin, F.-H: Singularly perturbed elliptic systems and multivalued harmonic functions with free boundaries. J. Am. Math. Soc. 21, 847-862 (2008)

55. Caginalp, G., Fife, P.C.: Dynamics of layered interfaces arising from phase boundaries. SIAM J. Appl. Math. 48, 506-518 (1988)

56. Cantrell, R.S., Cosner, C.: Spatial Ecology via Reaction-Diffusion Equations. Wiley, Chichester, (2003)

57. Carretero-González, R., Frantzeskakis, D.J., Kevrekidis, P.G.: Nonlinear waves in Bose-Einstein condensates: physical relevance and mathematical techniques. Nonlinearity 21, 139-202 (2008)

58. Chapman, S.J.: Superheating field of type II superconductors. SIAM J. Appl. Math. 55, 1233-1258 (1995)

59. Chen, X.: Spectrums for the Allen-Cahn, Cahn-Hilliard, and phase-field equations for generic interface. Commun. Partial Differ. Equ. 19, 1371-1395 (1994)

60. Chen, X., SAdhu, S.: Uniform asymptotic expansions of solutions of an inhomogeneous equation. J. Differ. Equ. 253, 951-976 (2012)

61. Chow, S.N., Hale, J.K.: Methods of Bifurcation Theory. Springer, Berlin, 1996

62. Coles, M.P., Pelinovsky, D.E., Kevrekidis, P.G.: Excited states in the ThomasFermi limit: a variational approach. Nonlinearity 23, 1753-1770 (2010)

63. Conti, M., Terracini, S., Verzini, G.: Asymptotic estimates for the spatial segregation of competitive systems. Adv. Math. 195, 524-560 (2005)

64. Coppel, W.A.: Stability and Asymptotic Behaviour of Differential Equations. Heath and Co, Boston, 1965

65. Correggi, M., Rougerie, N., Yngvason, J.: The transition to a giant vortex phase in a fast rotating Bose-Einstein condensate. Commun. Math. Phys. 303, 451-308 (2011)

66. Dafermos, C.M.: Solution of the Riemann problem for a class of hyperbolic systems of conservation laws by the viscosity method. Arch. Ration. Mech. Anal. 52, 1-9 (1973)

67. Dalfovo, F., Pitaevskit, L.,Stringari, S.: Order parameter at the boundary of a trapped Bose gas. Phys. Rev. A 54, 4213 (1996)

68. Dalfovo, F., Pitaevskit, L., Stringari, S.: The condensate wave function of a trapped atomic gas. J. Res. Natl. Inst. Stand. Technol. 101, 537 (1996)

69. DANCER, E.N., Du, Y.: Competing species equations with diffusion, large interactions, and jumping nonlinearities. J. Differ. Equ. 114, 434-475 (1994)

70. DANCER, E.N., YAN, S.: Construction of various types of solutions for an elliptic problem. Calc. Var. 20, 93-118 (2004)

71. Dancer, E.N., Yan, S.: On the superlinear Lazer-McKenna conjecture. J. Differ. Equ. 210, 317-351 (2005)

72. Dancer, E.N.: Stable and not too unstable solutions on $\mathbb{R}^{n}$ for small diffusion. In: Nonlinear Dynamics and Evolution Equations, Fields Institute Communications, vol. 48, pp. 67-93. American Mathematical Society, Providence, 2006

73. DANCER, E.N., YAN, S.: On the Lazer-Mckenna conjecture involving critical and supercritical exponents. Methods Appl. Anal. 15, 97-119 (2008) 
74. Dang, H., Fife, P.C., Peletier, L.A.: Saddle solutions of the bistable diffusion equation. Z. Angew. Math. Phys. 43, 984-998 (1992)

75. Daniels, P.G., LeE, A.T.: On the boundary-layer structure of patterns of convection in rectangular-planform containers. J. Fluid Mech. 393, 357-380 (1999)

76. De Boer, P.C.T., Ludford, G.S.S.: Spherical electric probe in a continuum gas. Plasma Phys. 17, 29-43 (1975)

77. DEL PINO, M.: Layers with nonsmooth interface in a semilinear elliptic problem. Commun. Partial Differ. Equ. 17, 1695-1708 (1992)

78. Del Pino, M., KowalczyK, M., WeI, J.: Concentration on curves for nonlinear Schrödinger equations. Commun. Pure Appl. Math. 60, 113-146 (2007)

79. Del Pino, M., KowalczyK, M., WeI, J.: The Toda system and clustering interface in the Allen-Cahn equation. Arch. Ration. Mech. Anal. 190, 141-187 (2008)

80. Del Pino, M., KowalczyK, M. Pacard, F., Wei, J.: The Toda system and multiple-end solutions of autonomous planar elliptic problems. Adv. Math. 224, 1462-1516 (2010)

81. del Pino, M., KowalczyK, M., Wei, J.: On De Giorgi conjecture in dimensions $N \geqq 9$. Ann. Math. 174, 1485-1569 (2011)

82. Del Pino, M. Musso, M., PaCARD, F.: Solutions of the Allen-Cahn equation invariant under screw-motion. Manuscr. Math. 138, 273-286 (2012)

83. Du, Q.: Diverse vortex dynamics in superfluids. Contemp. Math. 329, 105-117 (2003)

84. Du, Y., MA, L.: Logistic type equations on $\mathbb{R}^{N}$ by a squeezing method involving boundary blow-up solutions. J. Lond. Math. Soc. 64, 107-124 (2001)

85. Du, Y.: The heterogeneous Allen-Cahn equation in a ball: solutions with layers and spikes. J. Differ. Equ. 244, 117-169 (2008)

86. Du, Z., Gui, C.: Interior layers for an inhomogeneous Allen-Cahn equation. J. Differ. Equ. 249, 215-239 (2010)

87. Farina, A., SciunZi, B., Valdinoci, E.: Bernstein and De Giorgi type problems: new results via a geometric approach. Ann. Scuola Norm. Sup. Pisa Cl. Sci. VII, 741-791 (2008)

88. Felmer, P., Martinez, S.: High-energy solutions for a phase transition problem. $J$. Differ. Equ. 194, 198-220 (2003)

89. Felmer, P., Martínez, S., Tanaka, K.: Multi-clustered high-energy solutions for a phase transition problem. Proc. R. Soc. Edinb. Sect. A 135, 731-765 (2005)

90. Felmer, P., Mayorga-Zambrano, J.: Multiplicity and concentration for the nonlinear Schrödinger equation with critical frequency. Nonlinear Anal. 66, 151-169 (2007)

91. Felmer, P., Martínez, S., Tanaka, K.: Uniqueness of radially symmetric positive solutions for $-\Delta u+u=u^{p}$ in an annulus. J. Differ. Equ. 245, 1198-1209 (2008)

92. Fenichel, N.: Geometric singular perturbation theory for ordinary differential equations. J. Differ. Equ. 31, 53-98 (1979)

93. Fermi, E.: Statistical method of investigating electrons in atoms. Z. Phys. 48, 73-79 (1928)

94. Fetter, A.L., Feder, D.L.: Beyond the Thomas-Fermi approximation for a trapped condensed Bose-Einstein gas. Phys. Rev. A 58, 3185-3194 (1998)

95. FIfE, P.C.: Semilinear elliptic boundary value problems with small parameters. Arch. Ration. Mech. Anal. 52, 205-232 (1973)

96. FIFE, P.C., GREENLEe, M.W.: Interior transition layers of elliptic boundary value problems with a small parameter. Russ. Math. Surv. 29, 103-131 (1974)

97. FIFE, P.C.: A phase plane analysis of a corner layer problem arising in the study of crystalline grain boundaries (2004). Unpublished preprint, available online at http:// www.math.utah.edu/ fife

98. Floer, A., Weinstein, A.: Nonspreading wave packets for the cubic Schrödinger equation with a bounded potential. J. Funct. Anal. 69, 397-408 (1986)

99. FoKas, A.S., Its, A.R., KaPaev, A.A., NovoKshenov, V.Y.: Painlevé Transcendents, The Riemann-Hilbert Approach. American Mathematical Society, Providence, 2006 
100. Frantzeskakis, D.J., Theocharis, G., Diakonos, F.K., Schmelcher, P., Kivshar, Y.S.: Interaction of dark solitons with localized impurities in Bose-Einstein condensates. Phys. Rev. A 66, 053608 (2002)

101. Fusco, G., Pignotti, C.: Estimates for fundamental solutions and spectral bounds for a class of Schrödinger operators. J. Differ. Equ. 244, 514-554 (2008)

102. Fusco, G. Leonetti, F. Pignotti, C.: A uniform estimate for positive solutions of semilinear elliptic equations. Trans. Am. Math. Soc. 363, 4285-4307 (2011)

103. Gallo, C., Pelinovsky, D.: Eigenvalues of a nonlinear ground state in the ThomasFermi approximation. J. Math. Anal. Appl. 355, 495-526 (2009). See also the corresponding presentation available online at http://lmv.math.cnrs.fr/conferences-etcolloques/maths-physics-meeting/article/title-and-summary-of-talks

104. Gallo, C., Pelinovsky, D.: On the Thomas-Fermi ground state in a harmonic potential. Asymptot. Anal. 73, 53-96 (2011)

105. Gallo, C.: Expansion of the energy of the ground state of the Gross-Pitaevskii equation in the Thomas-Fermi limit. J. Math. Phys. 54, 031507 (2013)

106. Gesztesy, F., Jones, C.K.R.T., Latushkin, Y.,Stanislavova, M.: A spectral mapping theorem and invariant manifolds for nonlinear Schrödinger equations. Indiana Univ. Math. J. 49, 221-243 (2000)

107. Ghoussoub ,N., Gui, C.: On a conjecture of De Giorgi and some related problems. Math. Ann. 311, 481-491 (1998)

108. Gidas, B., Ni, W.M., Nirenberg, L.: Symmetry and related properties via the maximum principle. Commun. Math. Phys. 68, 209-243 (1979)

109. Gilbarg, D., Trudinger, N.S.: Elliptic Partial Differential Equations of Second Order, 2nd edn. Springer, New York, 1983

110. Grillakis, M., Shatah, J., Strauss, W.A.: Stability theory of solitary waves in the presence of symmetry I. J. Funct. Anal. 74, 160-197 (1987)

111. Grillakis, M.: Existence of nodal solutions of semilinear equations in $\mathbb{R}^{N}$. J. Differ. Equ. 85, 367-400 (1990)

112. GuedDA, M.: Note on the uniqueness of a global positive solution to the second Painlevé equation. Electron. J. Differ. Equ. 2001, 1-4 (2001)

113. Hastings, S.P., McLeOD, J.B.: A boundary value problem associated with the second Painlevé transcendent and the Korteweg-de Vries equation. Arch. Ration. Mech. Anal. 73, 31-51 (1980)

114. Hastings, S.P., Troy, W.C.: On some conjectures of Turcotte, Spence, Bau, and Holmes. SIAM J. Math. Anal. 20, 634-642 (1989)

115. Hastings, S.P., McLeod, J.B.: Periodic solutions of a forced second-order differential equation. J. Nonlinear Sci. 1, 225-245 (1991)

116. Hastings, S.P., McLeod, J.B.: Classical Methods in Ordinary Differential Equations. American Mathematical Society, Providence, 2012

117. Helffer, B., Weissler, F.B.: On a family of solutions of the second Painlevé equation related to superconductivity. Eur. J. Appl. Math. 9, 223-243 (1998)

118. Henry, D.: Geometric Theory of Semilinear Parabolic Equations. Lecture Notes in Mathematics, vol. 840. Springer, Heidelberg, 1981

119. Herring, G., Carr, L.D., Carretero-González, R., Kevrekidis, P.G., FRANTZESKAKIS, D.J.: Radially symmetric nonlinear states of harmonically trapped Bose-Einstein condensates. Phys. Rev. A 77, 023625 (2008)

120. Hervé, R.M., Hervé, M.: Etude qualitative des solutions réelles de l'équation différentielle $r^{2} f^{\prime \prime}(r)+r f^{\prime}(r)-q^{2} f(r)+r^{2} f(r)\left(1-f(r)^{2}\right)=0$. Ann. Inst. H. Poincaré Anal. Non Linéaire 11, 427-440 (1994)

121. Hislop, P.D., Sigal, I.M.: Introduction to Spectral Theory with Applications to Schrödinger Operators. Springer, New York, 1996

122. Holmes, P., Spence, D.: On a Painlevé-type boundary-value problem. Q. J. Mech. App. Math. 37, 525-538 (1984) 
123. Hutson, V., Lou, Y., Mischaikow, K.: Spatial heterogeneity of resources versus Lotka-Volterra dynamics. J. Differ. Equ. 185, 97-136 (2002)

124. Ichihara, R., Danshita, I., Nikuni, T.: Matter-wave dark solitons in a double-well potential. Phys. Rev. A 78, 063604 (2008)

125. Ignat, R., Millot, V.: The critical velocity for vortex existence in a two-dimensional rotating Bose-Einstein condensate. J. Funct. Anal. 233, 260-306 (2006)

126. Ignat, R., Millot, V.: Energy expansion and vortex location for a two-dimensional rotating Bose-Einstein condensate. Rev. Math. Phys. 18, 119-162 (2006)

127. IIDA, M., NAKashima, K., YANAGIDA, E.: On certain one-dimensionar elliptic systems under different growth conditions at respective infinities. In: Asymptotic Analysis and Singularities. Advanced Studies in Pure Mathematics, vol. 47-2, pp. 565-572, 2007

128. Iooss, G., JosePH, D.D.: Elementary Stability and Bifurcation Theory. Springer, New York, 1980

129. ITs, A.R., Novokshenov, V.Y.: The Isomonodromic Deformation Method in the Theory of the Painlevé Equations. Lecture Notes in Mathematics, vol. 1191, Springer, Heidelberg, 1986

130. JERRARD, R.L.: Local minimizers with vortex filaments for a Gross-Pitaevsky functional. ESAIM Control Optim. Calc. Var. 13, 35-71 (2007)

131. JonES, C.K.R.T.: Geometric singular perturbation theory. In: Johnson, R (ed.) Dynamical systems. Lecture Notes in Mathematics, vol. 1609, pp. 44-118. Springer, Heidelberg (1995)

132. Kabeya, Y., TANAKa, K.: Uniqueness of positive radial solutions of semilinear elliptic equations in $\mathbb{R}^{N}$ and Sérés non-degeneracy condition. Commun. Partial Differ. Equ. 24, 563-598 (1999)

133. Karali, G., Kevrekidis, P., Efremidis, N.: Nonlinear from linear states in twocomponent Bose-Einstein condensates. J. Phys. A 42, 045206 (2009)

134. Karali, G., Sourdis, C.: Radial and bifurcating non-radial solutions for a singular perturbation problem in the case of exchange of stabilities. Ann. Inst. H. Poincaré Anal. Non Linéaire 29, 131-170 (2012)

135. Karali, G., Sourdis, C.: Resonance phenomena in a singular perturbation problem in the case of exchange of stabilities. Commun. Partial Differ. Equ. 37, 1620-1667 (2012)

136. Kenig, C., NI, W.M.: An exterior Dirichlet problem with application to some nonlinear equations arising in geometry. J. Am. Math. Soc. 106, 689-702 (1984)

137. Kevrekidis, P.G., Pelinovsky, D.E.: Distribution of eigenfrequencies for oscillations of the ground state in the Thomas-Fermi limit. Phys. Rev. A 81, 023627 (2010)

138. Kevrekidis, P.G., Pelinovsky, D.E.: Variational approximations of trapped vortices in the large-density limit. Nonlinearity 24, 1271-1289 (2011)

139. Kirr, E., Kevrekidis, P.G., Pelinovsky, D.E.: Symmetry-breaking bifurcation in the nonlinear Schrödinger equation with symmetric potentials. Commun. Math. Phys. 308, 795-844 (2011)

140. Klingenberg, W.: Riemannian Geometry. Walter de Gruyter and Co, Berlin, 1982

141. Kohn, R.V., Sternberg, P.: Local minimizers and singular perturbations. Proc. $R$. Soc. Edinb. Sect. A 111, 69-84 (1989)

142. Kollár, R., Pego, R.L.: Spectral stability of vortices in two-dimensional BoseEinstein condensates via the Evans Function and Krein Signature. Appl. Math. Res. Express 2012, 1-46 (2012)

143. Konotop, V.V., Kevrekidis, P.G.: Bohr-Sommerfeld quantization condition for the Gross-Pitaevskii equation. Phys. Rev. Lett. 91, 230402 (2003)

144. KowalczyK, M., Liu, Y.: Nondegeneracy of the saddle solution of the Allen-Cahn equation. Proc. Am. Math. Soc. 139, 4319-4329 (2011)

145. Krupa, M., Szmolyan, P.: Extending geometric singular perturbation theory to nonhyperbolic points-fold and canard points in two dimensions. SIAM J. Math. Anal. 33, 286-314 (2001) 
146. Kurth, M.: On the existence of infinitely many modes of a nonlocal nonlinear Schrödinger equation related to dispersion-managed solitons. SIAM J. Math. Anal. 36, 967-985 (2004)

147. Kurata, K., Matsuzawa, H.: Multiple stable patterns in a balanced bistable equation with heterogeneous environments. Appl. Anal. 89, 1023-1035 (2010)

148. Lassoued, L., Mironescu, P.: Ginzburg-Landau type energy with discontinuous constraint. J. Anal. Math. 77, 1-26 (1999)

149. Levi, D., Winternitz, P. (eds.): Painlevé Transcendents: Their Asymptotics and Physical Applications. NATO ASI Series, Series B: Physics, vol. 278. Springer, New York (1990)

150. Li, G., Yang, J., Yan, S.: Solutions with boundary layer and positive peak for an elliptic Dirichlet problem. Proc. R. Soc. Edinb. Sect. A 134, 515-536 (2004)

151. Li, F., Nakashima, K.: Transition layers for a spatially inhomogeneous Allen-Cahn equation in multi-dimensional domains. Discrete Contin. Dyn. Syst. 32, 1391-1420 (2012)

152. Lieb, E.H., SeiRinger, R., YNGVAsOn, J.: A rigorous derivation of the Gross-Pitaevskii energy functional for a two-dimensional Bose gas. Commun. Math. Phys. 224, 17-31 (2001)

153. Lieb, E.H., Seiringer, R., Solovej, J.-P., Yngvason, J.: The Mathematics of the Bose Gas and Its Condensation. Book (2006). Avalable online at http://arxiv.org/abs/condmat/0610117v1

154. Lin, F.H., Lin, T.C.: Vortices in two-dimensional Bose-Einstein condensates. In: Geometry and Nonlinear Partial Differential Equations (Hangzhou, 2001), AMS/IP Studies in Advanced Mathematics, vol. 29, 87-114. American Mathematical Society, Providence, 2002

155. Liv, Z.: The spatial behavior of rotating two-component Bose-Einstein condensates. J. Funct. Anal. 261, 1711-1751 (2011)

156. Lundh, E., Pethick, C., Smith, H.: Zero-temperature properties of a trapped Bosecondensed gas: beyond the Thomas-Fermi approximation. Phys. Rev. A 55, 2126-2131 (1997)

157. MacKerrella, S.O., Blennerhassettb, P.J., Bassomc, A.P.: Görtler vortices in the Rayleigh layer on an impulsively started cylinder. Phys. Fluids 14, 2948-2956 (2002)

158. Madison, K., Chevy, F., Dalibard, J., Wohlleben, W.: Vortex formation in a stirred Bose-Einstein condensate. Phys. Rev. Lett. 84, 806 (2000)

159. Madison, K., Chevy, F., Dalibard, J., Wohlleben, W.: Vortices in a stirred BoseEinstein condensate. J. Mod. Opt. 47, 2715-2723 (2000)

160. Mahmoudi, F., Malchiodi, A., Wei, J.: Transition layer for the heterogeneous AllenCahn equation. Ann. Inst. H. Poincaré Anal. Non Linéaire 25, 609-631 (2008)

161. Margetis, D.: Asymptotic formula for the condensate wave function of a trapped Bose gas. Phys. Rev. A 61, 055601 (2000)

162. Margetis, D.: Bose-Einstein condensation beyond mean field: many-body bound state of periodic microstructure. SIAM Multiscale Model. Simul. 10, 383-417 (2012)

163. Montero, A.: Hodge decomposition with degenerate weights and the GrossPitaevskii energy. J. Funct. Anal. 254, 1926-1973 (2008)

164. Nakanishi, K., Schlag, W.: Invariant Manifolds and Dispersive Hamiltonian Evolution Equations. Zürich Lectures in Advanced Mathematics. European Mathematical Society, Zürich, 2011

165. Nakashima, K., Ni, W.-M., Su, L.: An indefinite nonlinear diffusion problem in population genetics, I: existence and limiting profiles. Discrete Contin. Dyn. Syst. 27, $617-641$ (2010)

166. Nefedov, N.N., SAкамото, K.: Multi-dimensional stationary internal layers for spatially inhomogeneous reaction-diffusion equations with balanced nonlinearity. $\mathrm{Hi}$ roshima Math. J. 33, 391-432 (2003) 
167. Noris, B., Terracini, S., Tavares, H., Verzini, G.: Uniform Hölder bounds for nonlinear Schrödinger systems with strong competition. Commun. Pure Appl. Math. 63, 267-302 (2010)

168. NI, W.M.: On the elliptic eqation $\Delta U+K U^{(n+2) /(n-2)}=0$, its generalization and application in geometry. Indiana Univ. Math. J. 4, 493-529 (1982)

169. OH, Y.-G.: Cauchy problem and Ehrenfest's law of nonlinear Schrödinger equations with potentials. J. Differ. Equ. 81, 255-274 (1989)

170. Ovchinnikov, Y.M., Sigal, I.M.: Ginzburg-Landau equation I. Static vortices. In: Partial Differential Equations and Their Applications. CRM Proceedings and Lecture Notes, vol. 12, pp. 199-220. American Mathematical Society, Providence, 1997

171. PaCard, F., Rivière, T.: Linear and Nonlinear Aspects of Vortices. The GinzburgLandau Model. Birkhaüser, Boston, 2000

172. Palamides, P.K., Galanis, G.N.: Positive, unbounded and monotone solutions of the singular second Painlevé equation on the half-line. Nonlinear Anal. 57, 401-419 (2004)

173. Pelinovsky, D.E., Kevrekidis, P.G.: Periodic oscillations of dark solitons in parabolic potentials. Contemp. Math. 473, 159-180 (2008)

174. Pelinovsky, D.: Asymptotic properties of excited states in the Thomas-Fermi limit. Nonlinear Anal. 73, 2631-2643 (2010)

175. Pelinovsky, D.E., Kevrekidis, P.G.: Bifurcations of asymmetric vortices in symmetric harmonic traps. Appl. Math. Res. Express 2013, 127-164 (2013)

176. Pethick, C., Smith, H.: Bose-Einstein Condensation in Dilute Gases. Cambridge University Press, Cambridge, 2002

177. Pitaevskit, L., Stringari, S.: Bose-Einstein Condensation. Oxford University Press, Oxford, 2003

178. Rabinowitz, P.H.: On a class of nonlinear Schrödinger equations. Z. Angew. Math. Phys. 43, 270-291 (1992)

179. REBAÏ, Y.: Weak solutions of nonlinear elliptic equations with prescribed singular set. J. Differ. Equ. 127, 439-453 (1996)

180. Rougerie, N.: Vortex rings in fast rotating Bose-Einstein condensates. Arch. Ration. Mech. Anal. 203, 69-135 (2012)

181. Ryu, C., et al.: Observation of persistent flow of a Bose-Einstein condensate in a toroidal trap. Phys. Rev. Lett. 99, 260-401 (2007)

182. Salman, H.: Approximating steady states in equilibrium and nonequilibrium condensates. Phys. Rev. A 85, 063622 (2012)

183. Sandier, E., Serfaty, S.: Vortices in the Magnetic Ginzburg-Landau Model. Birkhäuser, Boston, 2006

184. Sattinger, D.H.: Topics in Stability and Bifurcation Theory. Lecture Notes in Mathematics, vol. 309. Springer, Heidelberg, 1973

185. Seiringer, R.: Gross-Pitaevskii theory of the rotating Bose gas. Commun. Math. Phys. 229, 491-509 (2002)

186. Seiringer, R.: Dillute, trapped Bose gases and Bose-Einstein condensation. Lect. Notes Phys. 695, 249-274 (2006)

187. Selem, F.H.: Radial solutions with prescribed numbers of zeros for the nonlinear Schrödinger equation with harmonic potential. Nonlinearity 24, 1795-1819 (2011)

188. Serfaty, S.: On a model of rotating superfluids. ESAIM Control Optim. Calc. Var. 6, 201-238 (2001)

189. Schecter, S.: Existence of Dafermos profiles for singular shocks. J. Differ. Equ. 205, 185-210 (2004)

190. Schecter, S., Sourdis, C.: Heteroclinic orbits in slow-fast Hamiltonian systems with slow manifold bifurcations. J. Dyn. Differ. Equ. 22, 629-655 (2010)

191. Sourdis, C., Fife, P.C.: Existence of heteroclinic orbits for a corner layer problem in anisotropic interfaces. Adv. Differ. Equ. 12, 623-668 (2007)

192. Stock, S., Bretin, V., Chevy, F., Dalibard, J.: Shape oscillation of a rotating BoseEinstein condensate. Europhys. Lett. 65, 594 (2004) 
193. Sulem, C., Sulem, P.L.: The Nonlinear Schrödinger Equation. Springer, New York, 1999

194. Sun, J.: An equation for the limit state of a superconductor with pinning sites. Electron. J. Differ. Equ. 2005, 1-24 (2005)

195. Thomas, L.H.: The calculation of atomic fields. Proc. Camb. Philos. Soc. 23, 542 (1927)

196. Tsai, T.-P., YaU, H.-T.: Asymptotic dynamics of nonlinear Schrödinger equations: resonance-dominated and dispersion-dominated solutions. Commun. Pure Appl. Math. 55, 153-216 (2002)

197. Turcotte, D.L., Spence, D.A., Bau, H.H.: Multiple solutions for natural convective flows in an internally heated, vertical channel with viscous dissipation and pressure work. Int. J. Heat Mass Transf. 25, 699-706 (1982)

198. VAn Dyke, M.: Perturbation Methods in Fluid Mechanics. Academic Press, New York, 1964

199. WeI, J., Weth, T.: Asymptotic behavior of solutions of planar elliptic systems with strong competition. Nonlinearity 21, 305-317 (2008)

200. WeI, J., YANG, J.: Solutions with transition layer and spike in an inhomogeneous phase transition model. J. Differ. Equ. 246, 3642-3667 (2009)

201. WEI, J., YANG, J.: Toda system and cluster phase transition layers in an inhomogeneous phase transition model. Asymptot. Anal. 69, 175-218 (2010)

202. WeI, J., Lin, T.C., YAng, J.: Vortex rings for the Gross-Pitaevskii equation in $\mathbb{R}^{3}$. J. Math. Pures Appl. 100, 69-112 (2013)

203. WeI, J., YAng, J.: Vortex rings pinning for the Gross-Pitaevskii equation in three dimensional space. SIAM J. Math. Anal. 44, 3991-4047 (2012)

204. Weiler, C.N., et al.: Spontaneous vortices in the formation of Bose-Einstein condensates. Nature 455, 948 (2008)

205. Wu, T.T.: Bose-Einstein condensation in an external potential at zero temperature: general theory. Phys. Rev. A 58, 1465-1474 (1998)

206. Yan, D., Kevrekidis, P.G., Frantzeskakis, D.J.: Dark solitons in a Gross-Pitaevskii equation with a power-law nonlinearity: application to ultracold Fermi gases near the Bose-Einstein condensation regime. J. Phys. A 44, 415202 (2011)

207. YIN, H., ZHANG, P.: Bound states of nonlinear Schrödinger equations with potentials tending to zero at infinity. J. Differ. Equ. 247, 618-647 (2009)

208. Zhang, J.: Stability of standing waves for nonlinear Schrödinger equations with unbounded potentials. Z. Angew. Math. Phys. 51, 498-503 (2000)

209. Zhou, L., Xu, H., Liu, Z.: Asymptotic behavior of critical points for a Gross-Pitaevskii energy. Nonlinear Anal. 74, 4274-4291 (2011)

Department of Mathematics and Applied Mathematics,

University of Crete,

Heraklion,

Greece.

e-mail: sourdis@uoc.gr

e-mail: gkarali@tem.uoc.gr

e-mail: csourdis@tem.uoc.gr

(Received June 3, 2012 / Accepted December 15, 2014)

Published online February 3, 2015 - C Springer-Verlag Berlin Heidelberg (2015) 